Article

\title{
New Insights into the LGM and LG in Southern France (Vaucluse): The Mustelids, Micromammals and Horses from Coulet des Roches
}

\author{
Evelyne Crégut-Bonnoure $^{1, *}$, Nicolas Boulbes ${ }^{2}$, Emmanuel Desclaux $^{3}$ (D) and Adrian Marciszak ${ }^{4}$ \\ 1 Muséum Requien 67, Rue Joseph-Vernet 84000 Avignon and TRACES, UMR 5608 (CNRS-Université \\ Toulouse le Mirail), 5 Allées Antonio Machado, 31058 Toulouse, CEDEX 9, France \\ 2 HNHP, UMR 7194/Université de Perpignan, Centre Européen de Recherche Préhistorique 1, Avenue Léon \\ Jean Grégory, 66720 Tautavel, France; nicolas.boulbes@cerptautavel.com \\ 3 Laboratoire Départemental de Préhistoire du Lazaret, CEPAM, UMR 7264 CNRS, 33bis Boulevard F. Pilatte, \\ 06300 Nice, France; emmanuel.desclaux@gmail.com \\ 4 Department of Paleozoology, Institute of Environmental Biology, Faculty of Biological Sciences, \\ University of Wrocław, Sienkiewicza 21,50-335 Wrocław, Poland; amarcisz@biol.uni.wroc.pl or \\ adrian.marciszak@uwr.edu.pl \\ * Correspondence: evelyne.cregut@orange.fr
}

Academic Editors: Juan Manuel López García and Maria Rita Palombo Received: 30 May 2018; Accepted: 17 September 2018; Published: 27 September 2018

\begin{abstract}
Coulet des Roches is a natural karst trap in Southern France. Its infilling dates back to the end of the Pleniglacial (Last Glacial Maximum, LGM) and the end of the Tardiglacial (Last Glacial, LG). Three mustelid species have been identified in this infilling: the common polecat (Mustela putorius, minimum number of individuals $(\mathrm{MNI})=4)$, the stoat (Mustela erminea, $\mathrm{MNI}=14$ ) and the weasel (Mustela nivalis, MNI $=48$ ). The common polecat remains are metrically and morphologically indistinguishable from recent European specimens. The smallest mustelids are mainly represented by average-sized specimens, which are slightly smaller than extant species. A partial weasel skeleton of an extremely small pygmy weasel, regarded as a typical glacial element, was also discovered. Sexual dimorphism is strongly pronounced. Seventeen horses have been identified, corresponding to the chrono subspecies Equus ferus gallicus. The analysis of the muzzles and metapodials shows overall adaptation to cool and dry weather conditions. The ibexes are typical of Capra ibex $(\mathrm{MNI}=15)$. The $\mathrm{p} 3$ morphology is similar to the LGM populations located on the southern side of the Durance River, with an important dilation of the metaconid, except for the oldest LGM specimen. This dilation is older on the southern side of the Durance River, as it occurs at the end of MIS 3. This difference could reflect the barrier role of the Durance River. Micromammals are abundant (mostly related to rodents and shrews; 18 genera/species; $\mathrm{MNI}=470$ ). The paleoecological study highlighted important and rapid climatic and environmental fluctuations throughout the sequence. As a result of climatic fluctuations, the plains constituted a corridor for the migration of temperate species to Provence during cold periods ("southern refuge zone") and their re-immigration to Western Europe during temperate episodes. In a related and complementary way, the highland areas not only played a natural role as a geographical barrier, but also constituted a refuge zone during interglacial episodes for some micromammal species originating from northern and eastern parts of Europe ("cryptic southern refugia").
\end{abstract}

Keywords: south-eastern France; Last Glacial Maximum; mustelids; horses; ibexes; micromammals; ecological adaptation 


\section{Introduction}

The end of the Late Pleistocene is a well-known cold period in Western Europe and is referred to as the Last Glacial Maximum (LGM). It extends from 23,000 to 14,000 cal BP, with a mid-point at 18,000 cal BP [1]. This period is marked by important environmental modifications in relation to the furthest extension of the ice sheets. These harsh climatic conditions progressively diminished towards the Holocene. The study of ice, marine and terrestrial cores (Greenland Stadial (GS) and Interstadial (GI); Dansgaard-Oeschger stages (DO); Heinrich Stadial (HS)) has identified several well-defined climatic oscillations. Up until now, in the east of Southern France, and more precisely in the Provence area, data relating to the environmental transformation from the LGM to the climatic optimum were confined to several archaeological sites and our knowledge of the fauna was limited to the large mammals hunted by man. Recently, several small pits, about ten feet deep, were discovered near the Vaucluse highlands [2] (Figure 1). They acted as natural traps for the local fauna. The Coulet des Roches hole is one of them. Excavations begun there in 2007 and are still in progress. Complete skeletons of mustelids, horses, ibexes and micromammals from the Pleistocene deposits enable us to assess the adaptation of each species in relation to the climate, as well as the phenotypic expressions. They provide new information on the paleoenvironment and climatic fluctuations from $23,430 \pm 80$ cal BP to the Holocene.

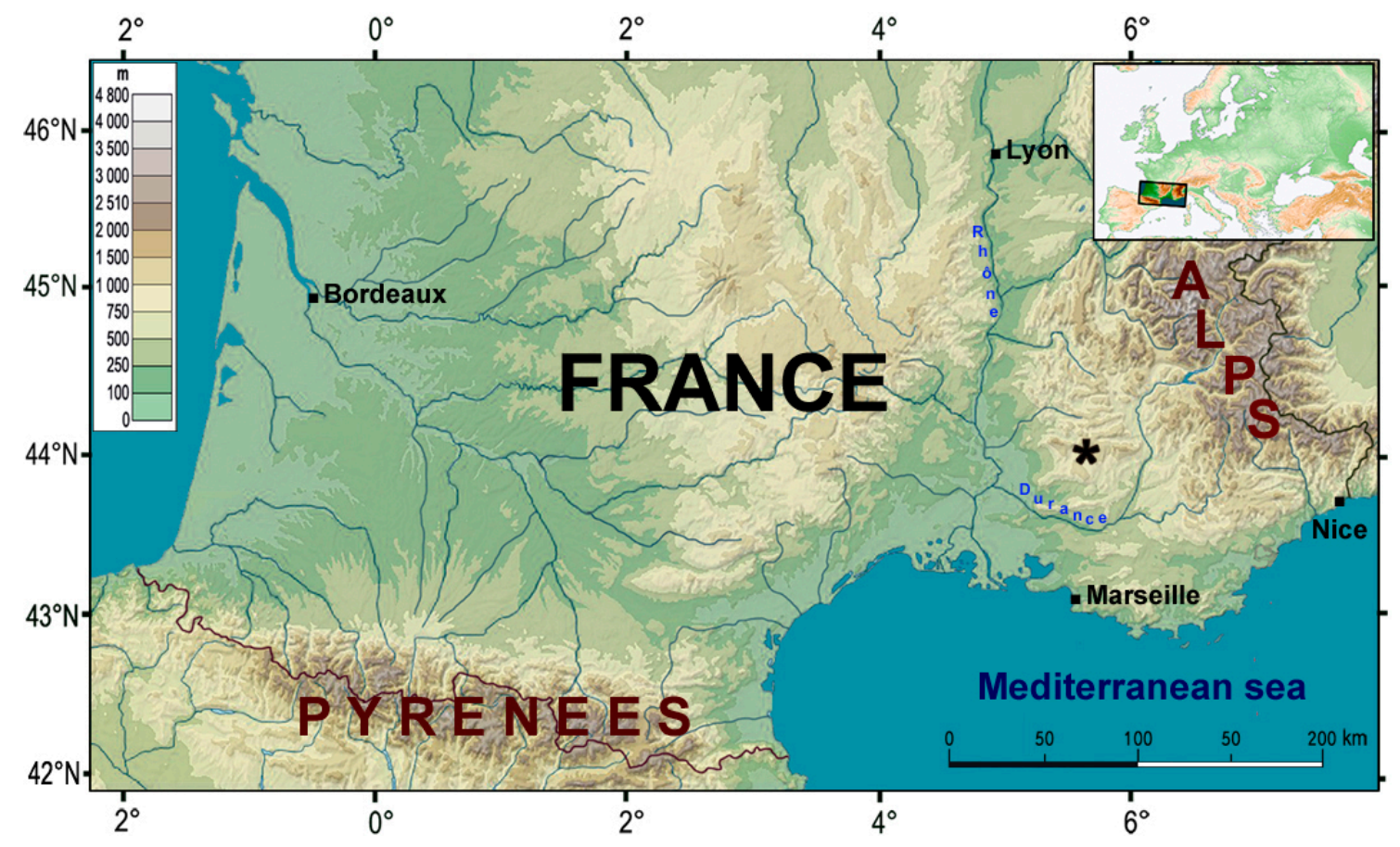

Figure 1. Location of Coulet des Roches. Map modified from Geoatlas.

The material is stored in the collections of the Natural History Museum of Avignon (Requien Museum).

\section{Presentation of Coulet des Roches (ECB)}

Coulet des Roches is a vertical hole with an ellipsoidal opening of about $4 \mathrm{~m} \times 2 \mathrm{~m}$, located at an altitude of $700 \mathrm{~m}$. It widens instantly until the current depth of $14 \mathrm{~m}$, directly below the opening and attains a depth of $13 \mathrm{~m}$ on the southern side (Figure 2). At the bottom, there is a large 10-m-long chamber, with an average width of 3-4 m. It was opened by spelunkers from Carpentras in 1971. They stopped their investigation in 1973 [3-8]. Our investigations began in 2007 and are still in progress [2,9-17]. 


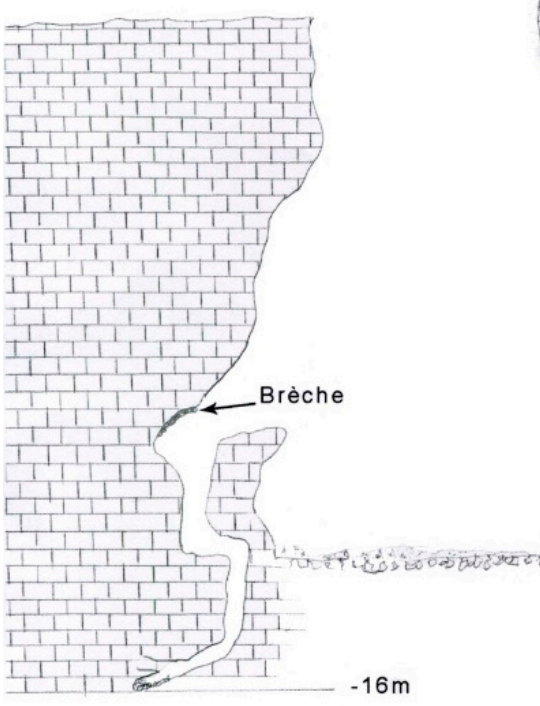

$0=5 \mathrm{M}-(1.00)-\left(100^{\circ}, 20^{\circ}\right)$

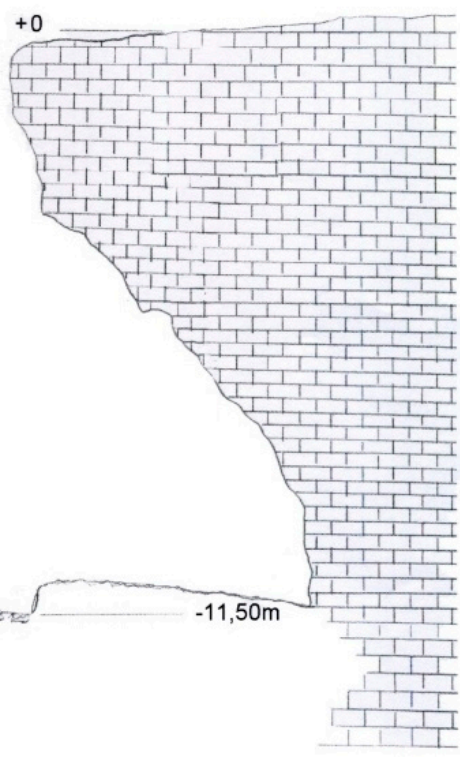

Le 11décembre 2015 (DISTO X310)

Figure 2. Coulet des Roches. Cross section. Topographical survey: Gandelet S. and Bérard Ch.

The sediments extracted by the spelunkers from 1971 to 1973 were screened, and contained modern, Holocene (Atlantic and Subboreal periods [2,18]) and Pleistocene species. At the bottom of the hole, a sequence comprising three main stratigraphic units was identified [2]. Today, five stratigraphic units have been defined (US) [17]. Stalagmitic floors and flowstones are visible on the walls. The stalagmitic floors indicate important flows of water at two distinct periods of the Middle Pleistocene: during MIS 13 and MIS 11.

Radiocarbon dating of numerous bones and charcoals, paleontological and palynological data date the Coulet infillings to the end of the Pleistocene (LGM; 23,430 $\pm 80 \mathrm{cal} \mathrm{BP}$ ) and the end of the Holocene (Subboreal; $3610 \pm 40$ cal BP).

\section{The LGM and LG Infillings (ECB)}

The LGM and Last Glacial (LG) sequence is represented primarily by US3. This unit contains homogeneous cryoclastic sediment formed by the accumulation of small-sized graded limestone fragments. It is about $4 \mathrm{~m}$ thick. According to the dating of the large mammals, the top of the unit is contemporaneous with GS 2a. Currently, at a depth of 6-7 m, the LGM cryoclastic sediment tends to disappear. It is replaced in the southeastern corner of the chamber by a clay layer which covers one of the horses dated to 26,700-26,250 cal BP, using the IntCal09 curve [19]. It is correlated with GI 3 ("Tursac" episode) and overlies important scree. In the mid-eastern sector of the chamber, the cryoclastic limestones are smaller and corroded by significant water circulation (US5). They can be correlated to GI 4 ("Maisières" interstadial).

In a sector located at the base of the south-western corner of the chamber, an earthy layer (US4) is dated to GI 10-11 ("Hengelo" interpleniglacial; MIS 3), on the basis of a Cervus elaphus radiocarbon date [2]. It seems to result from the draining of a crack. However, it could represent a partially destroyed infilling or karstic suffosion.

\section{Paleontology}

The LGM and LG fauna is represented by large and small mammals and birds. At least 48 species are present in this Pleistocene sequence: 11 large mammals, 2 lagomorphs, 5 insectivores, 12 rodents, 17 birds, and 1 reptile (Table 1 ). 
Table 1. Distribution of the species during GI 10-11, GI 3, Last Glacial Maximum (LGM) and Last Glacial-GS 2a.

\begin{tabular}{|c|c|c|c|c|}
\hline & GI 10-11 & GI 3 & LGM & LG/GS 2a \\
\hline \multicolumn{5}{|l|}{ Mammals } \\
\hline Vulpes vulpes & - & - & $X$ & $X$ \\
\hline Alopex lagopus & - & - & $x$ & $x$ \\
\hline Mustela putorius & - & - & $x$ & $X$ \\
\hline Mustela erminea & - & $x$ & $x$ & $x$ \\
\hline Mustela nivalis & $x$ & $X$ & $x$ & $x$ \\
\hline Equus ferus gallicus & - & $X$ & $x$ & $x$ \\
\hline Cerous elaphus & $x$ & - & - & - \\
\hline Rangifer tarandus & - & - & $x$ & $x$ \\
\hline Capra ibex & - & - & $X$ & $X$ \\
\hline Rupicapra rupicapra & - & - & $x$ & $x$ \\
\hline Rupicapra sp. & - & - & $X$ & - \\
\hline Lepus timidus & - & $x$ & $x$ & $x$ \\
\hline Oryctolagus cuniculus & - & - & $X$ & $X$ \\
\hline Talpa europaea & - & - & $x$ & $x$ \\
\hline Crocidura leucodon/russula & - & - & $x$ & $x$ \\
\hline cf. Sorex sp. & - & - & - & $X$ \\
\hline Sorex minutus & - & - & $x$ & - \\
\hline Sorex gr. araneus/coronatus & - & - & $x$ & - \\
\hline Marmota marmota primigenia & - & - & $X$ & $X$ \\
\hline Eliomys quercinus & - & - & $x$ & $x$ \\
\hline Apodemus sylvaticus & - & - & $x$ & - \\
\hline Arvicola amphibius & - & - & $x$ & - \\
\hline Microtus (Stenocranius) gregalis & - & - & $X$ & - \\
\hline Microtus arvalis & - & - & $x$ & $X$ \\
\hline Microtus arvalis/agrestis & - & - & - & $x$ \\
\hline Chionomys nivalis & - & - & $x$ & $x$ \\
\hline Clethrionomys glareolus & - & - & $x$ & $x$ \\
\hline Microtus (Terricola) cf. multiplex & - & - & $x$ & - \\
\hline Microtus oeconomus & - & - & $X$ & $x$ \\
\hline Dicrostonyx torquatus & - & - & $X$ & $X$ \\
\hline \multicolumn{5}{|l|}{ Birds } \\
\hline Aquila chrysaetos & - & - & $X$ & $x$ \\
\hline Falco cf. peregrinus & - & - & $x$ & $x$ \\
\hline Falco tinnunculus & - & - & $x$ & - \\
\hline Circus sp. & - & - & $x$ & - \\
\hline Bubo scandiacus & - & - & $x$ & $x$ \\
\hline Lagopus mutus & - & - & $X$ & - \\
\hline Pyrhocorax pyrhocorax & - & - & $X$ & $X$ \\
\hline Pyrhocorax graculus & - & - & $X$ & $X$ \\
\hline Corvus monedula & - & - & $X$ & $x$ \\
\hline Pyrrhocorax/Corvus & - & - & $x$ & $x$ \\
\hline Garrulus glandarius & - & - & $x$ & - \\
\hline Columba oenas & - & - & - & $x$ \\
\hline Columba livia & - & - & $x$ & $X$ \\
\hline Columba livia-oenas & - & - & $x$ & $x$ \\
\hline cf. Nucifraga caryocatactes & - & - & $x$ & - \\
\hline Rallus aquaticus & - & - & - & $x$ \\
\hline Hirundo sp. & - & - & - & $x$ \\
\hline \multicolumn{5}{|l|}{ Reptile } \\
\hline Emys orbicularis & $x$ & - & - & - \\
\hline
\end{tabular}

This faunal assemblage is unprecedented in the east of Southern France. Some of the Mustelid, Caprine and Equid species reflect ecological adaptation and provide new evidence of the phenotypic 
plasticity of these taxa. Micromammals highlight important and rapid climatic and environmental fluctuations perceptible throughout the sequence.

\subsection{Mustelids ( $A M$ and $E C B$ )}

Three mustelid species were identified (ECB): the common polecat (Mustela putorius, minimum number of individuals $(\mathrm{MNI})=4)$, the stoat $($ Mustela erminea, $\mathrm{MNI}=14)$ and the weasel (Mustela nivalis, MNI = 48) [17]. Point to point measurements were taken by one of us (ECB), with an electronic caliper to the nearest $0.01 \mathrm{~mm}$. The measurements shown in brackets correspond to the estimated value of the dimensions. Osteological and dental terminology follows Anderson [20]. The measurements are shown in Figures 3-5.
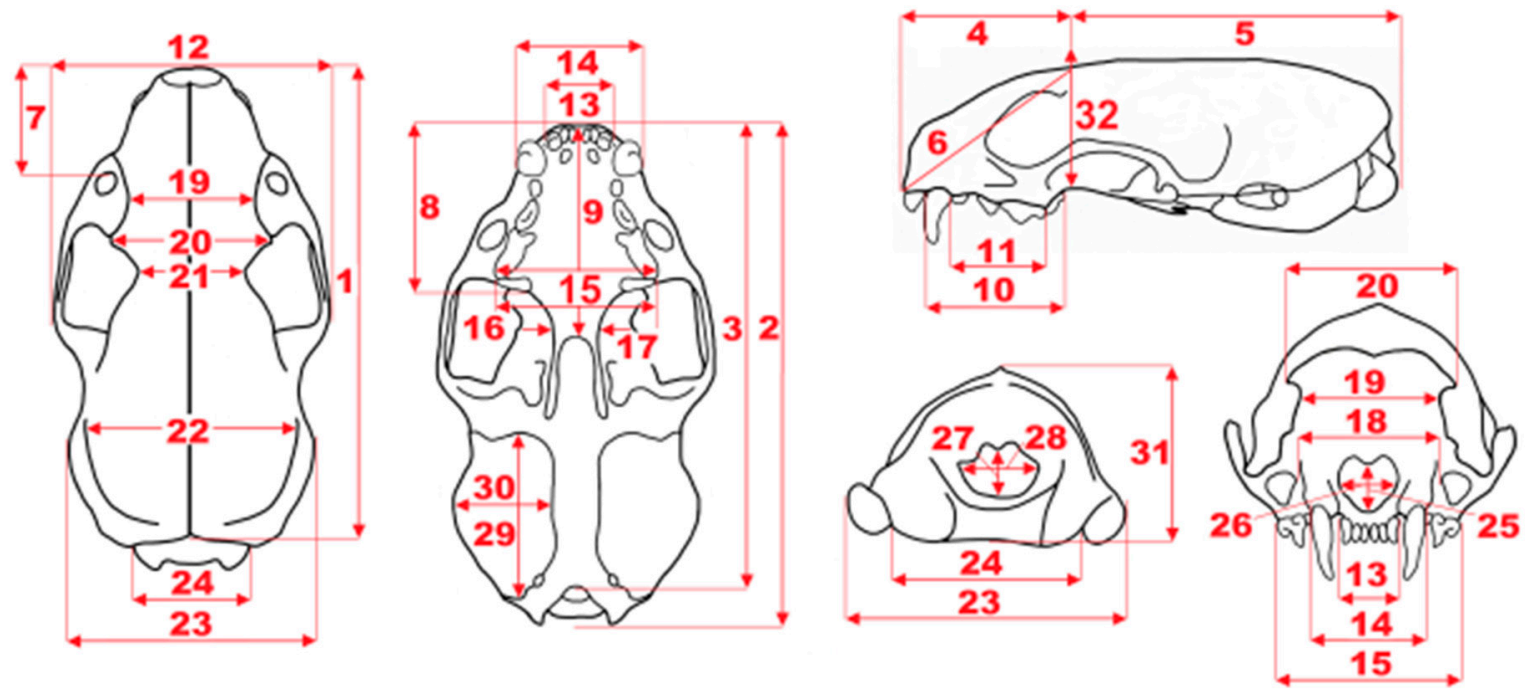

Figure 3. Scheme of measurements of mustelid calvarium: 1, total length (prosthion-acrocranion); 2, condylobasal length (prosthion-occipital condyles); 3, basal length (prosthion-basion); 4, viscerocranium length (prosthion-point F); 5, neurocranium length (point F-acrocranion); 6, facial length (prosthion-point F); 7, rostrum length (prosthion-infraorbital); 8, rostrum length (anterior margin of I1 to posterior margin of M1); 9, palatal length (prosthion-staphylion); 10, C1-M1 length on alveoli (anterior margin of $\mathrm{C} 1$ alveolus to the posterior margin of M1 alveolus); 11, upper premolar row length on alveoli (anterior margin of P2 alveolus to the posterior margin of P4 alveolus); 12 , breadth at zygomatic arches (zygion-zygion); 13, incisor row breadth (I3-I3 breadth); 14, maximal breadth at the canine alveoli; 15 , maximal breadth at the P4 alveoli; 16 , maximal breadth at the M1 alveoli; 17 , lowest palatal breadth; 18 , lowest breadth between infraorbital foramina; 19 , lowest breadth between orbits (entorbital-entorbital); 20, frontal breadth (ectorbital-ectorbital); 21, postorbital lowest breadth (postorbital bar); 22, maximal neurocranium breadth (euryon-euryon); 23, mastoid breadth (otion-otion); 24, maximal breadth of occipital condyles; 25, nasal aperture height; 26, nasal aperture breadth; 27, height of foramen magnum (basionopisthion); 28, breadth of foramen magnum; 29, bullae ossae length; 30, bullae ossae breadth; 31, cranial height (acrocranion-basion); 32, maximal cranial height (staphylion-frontal). 


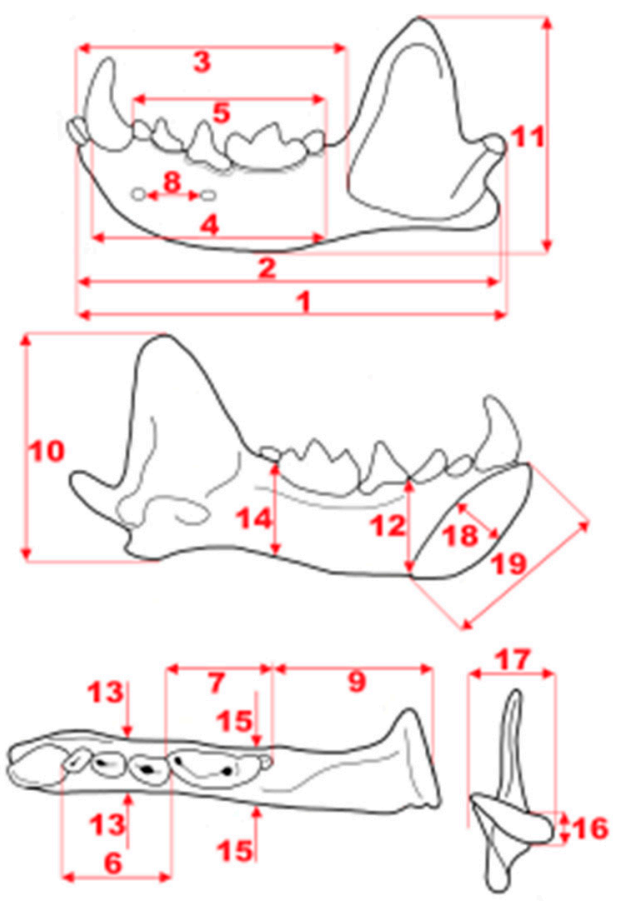

Figure 4. Scheme of measurements of mustelid mandible: 1, total length (condyle to infradentale); 2 , distance of angular process to infradentale; 3 , distance of infradentale to anterior margin of masseter fossa; 4 , distance of anterior margin of $\mathrm{c} 1$ to posterior margin of $\mathrm{m} 2 ; 5$, cheek teeth row length (anterior margin of $\mathrm{p} 1$ to posterior margin of $\mathrm{m} 2$ ); 6 , premolar row length (anterior margin of $\mathrm{p} 1$ to posterior margin of $\mathrm{p} 4$ ); 7 , molar row length (anterior margin of $\mathrm{m} 1$ to posterior margin of $\mathrm{m} 2$ ); 8 , distance between mental foramina; 9 , distance of posterior margin of $\mathrm{m} 2$ to condyle; 10 , distance of angular process to coronoid process; 11 , mandible maximum height; 12 , mandible body height between $\mathrm{p} 3$ and p4; 13, mandible body thickness between $\mathrm{p} 3$ and p4; 14, mandible body height between $\mathrm{m} 1$ and $\mathrm{m} 2 ; 15$, mandible body thickness between $\mathrm{m} 1$ and $\mathrm{m} 2 ; 16$, condyle height; 17 , condyle breadth; 18 , symphysis maximum diameter; 19 , symphysis minimum diameter.
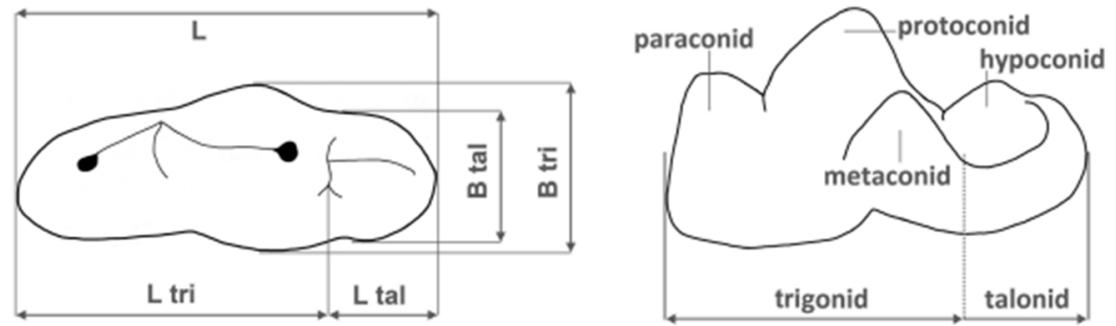

Figure 5. Lower carnassial (m1) measurements (left) and cusp terminology (right). L, total length; L tri, trigonid length; L tal, talonid length; B tri, trigonid breadth; B tal, talonid breadth; tri, trigonid; tal, talonid; par, paraconid; pro, protoconid; met, metaconid; hyp, hypoconid.

\subsubsection{Mustela putorius Linnaeus, 1758}

Species diagnosis

Detailed metric and morphological analyses carried out by many previous authors and recently critically revisited by Marciszak [20] show that two polecat species can be distinguished, based in particular on cranial material. Compared to Mustela eversmanii, Mustela putorius differs in the following ways (for indexes the mean was given) (Figures 6 and 7) [21-43].

1. Narrower spacing of zygomatic arches: index of zygomatic arch breadth to the condylobasal skull length is 64.9 in M. eversmanii while in M. putorius it is 56.7. 
2. Broader rostrum on canines: the index of $\mathrm{C} 1-\mathrm{C} 1$ breadth to the condylobasal skull length in M. putorius is 24.7, while in M. eversmanii it is 31.4 .

3. Broader rostrum on carnassials: the index of P4-P4 breadth to the condylobasal skull length in M. putorius is 34.8 , while in M. eversmanii it is 39.8 .

4. Rectangular and broad postorbital bar: while in M. eversmanii this constriction is triangular and much narrower, the index of postorbital bar breadth to the condylobasal skull length in M. putorius is 24.2 , while in M. eversmanii it is 20.2 .

5. Oval nasal aperture: where height exceeds breadth, in M. eversmanii, nasal aperture is rounded, the value of the height is comparable to the breadth, the index of breadth to the height of the nasal aperture in M. putorius is 85.1, while in M. eversmanii, it is 108.9 .

6. Nasal bones are proportionally longer and triangular.

7. Muscle attachments are less developed: the sagittal crest is shorter, lower and less developed.

8. The palatal posterior part is broader, V-shaped with well-marked curvature of posterior edges, the index of postorbital bar breadth to the condylobasal skull length in M. putorius is 12.5, while in M. eversmanii it is 10.6.

9. Narrower spacing of the tympanic bullae: the index of breadth between them to the condylobasal skull length is 44.4 in M. putorius, while in M. eversmanii, it is 50.3 .

10. Proportionally smaller orbits: the index of orbit height to the condylobasal skull length in M. putorius is 15.1, while in M. eversmanii it is 15.9.

11. Narrower incisor row: the index of I3-I3 breadth to the condylobasal skull length in M. putorius is 10.3 , while in M. eversmanii it is 11.9 .

12. Proportionally shorter and narrower upper canines: the index of $\mathrm{C} 1$ breadth to $\mathrm{C} 1$ length in M. putorius is 71.7 , while in M. eversmanii it is 76.4 .

13. Less reduced P2: the index of $\mathrm{P} 2$ length to $\mathrm{P} 3$ length in M. putorius is 59.6, while in M. eversmanii it is 52.2 .

14. Proportionally smaller P4: the index of P3 length to P4 length in M. putorius is 55.9, while in M. eversmanii it is 53.8 .

15. Less reduced M1: the index of M1 length to P4 length in M. putorius is 82.2, while in M. eversmanii it is 65.1.

16. Less reduced lingual margin of M1: the index of M1 lingual margin length to P4 length in M. putorius is 44.4, while in M. eversmanii it is 34.1 .

17. Lower, narrower and less massive mandible body: the index of M1 length to the height of mandible body after $\mathrm{m} 1$ in M. putorius is 110.0 , while in M. eversmanii it is 92.1 .

18. Masseter fossa is shorter and shallower: anterior margin is rounded and reaches $\mathrm{m} 1 / \mathrm{m} 2$ border, the index of mandible length measured to the anterior margin of the masseter fossa to mandible total length in M. putorius is 63.6, while in M. eversmanii it is 69.0.

19. The mandible ramus is higher: less massive and its margins create a more open angle.

20. The condylar process is usually located below tooth level.

21. Proportionally shorter and narrower lower canines: the index of $\mathrm{c} 1$ breadth to $\mathrm{c} 1$ length in M. putorius is 69.9 , while in M. eversmanii it is 79.1 .

22. Less reduced $\mathrm{p} 2$ : the index of $\mathrm{p} 2$ length to $\mathrm{p} 4$ length in $M$. putorius is 53.9, while in M. eversmanii it is 49.7 .

23. Less broadened posterior part of $\mathrm{p} 4$ crown: the index of $\mathrm{p} 4$ posterior breadth to $\mathrm{p} 4$ length in M. putorius is 60.7, while in M. eversmanii it is 68.3 .

24. Proportionally smaller and narrower $\mathrm{m} 1$ : the index of $\mathrm{p} 4$ length to $\mathrm{m} 1$ length in M. putorius is 52.4, while in M. eversmanii it is 45.8 .

25. Less reduced talonid of $\mathrm{m} 1$ : the index of $\mathrm{m} 1$ talonid length to $\mathrm{m} 1$ trigonid length in M. putorius is 25.8 , while in M. eversmanii it is 21.7 , the index of $\mathrm{m} 1$ talonid breadth to $\mathrm{m} 1$ trigonid breadth in M. putorius is 71.0, while in M. eversmanii it is 62.6 . 
26. Less reduced $\mathrm{m} 2$ : the index of $\mathrm{m} 2$ length to $\mathrm{m} 1$ length in M. putorius is 27.4 , while in M. eversmanii it is 20.2 .
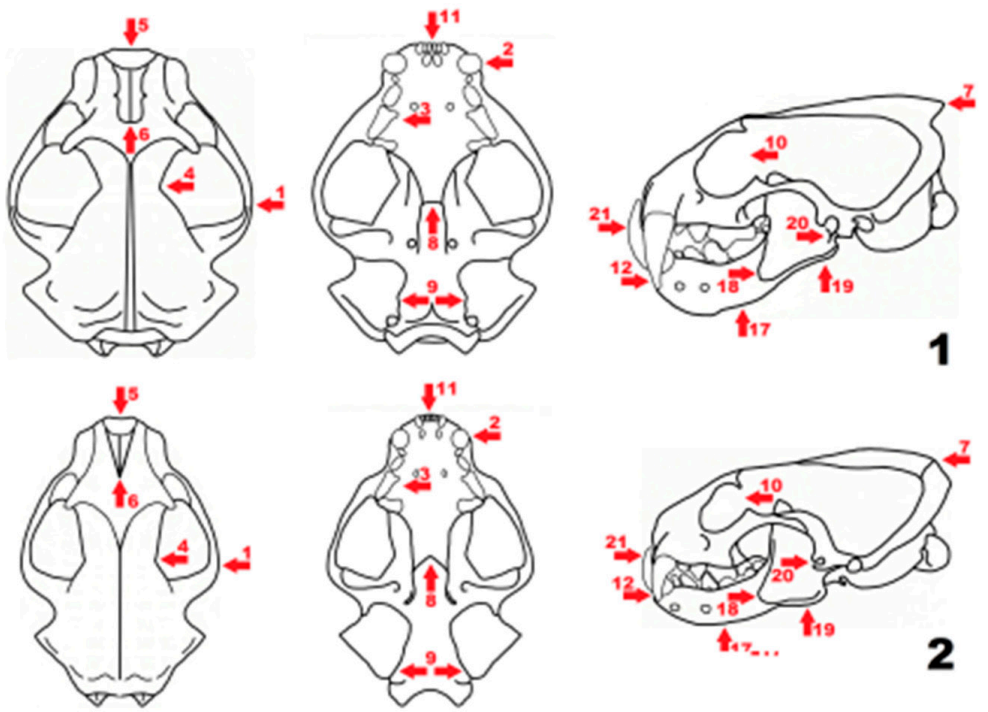

Figure 6. Schematic presentation of cranial features distinguishing Mustela eversmanii (1) from Mustela putorius (2). The features are indicated by red arrows, the numbers next to them correspond to the number of characteristics described in the text.
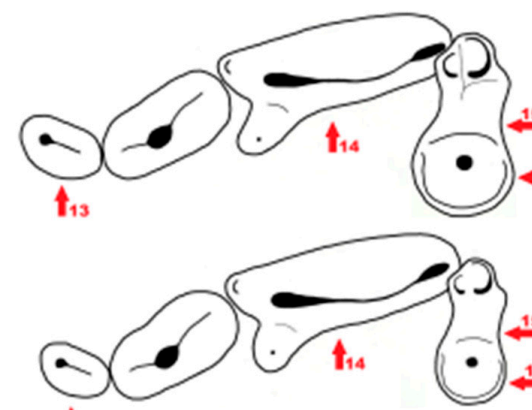

$\mathbf{t}_{13}$
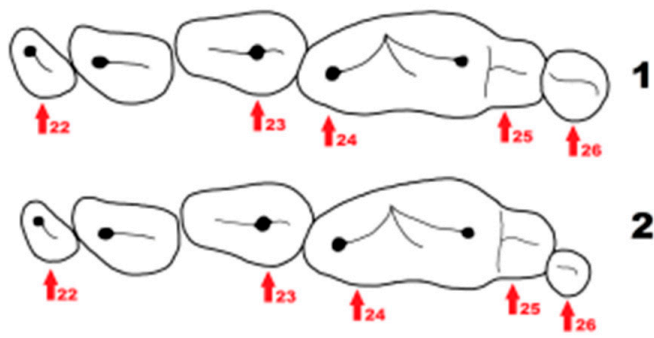

2

Figure 7. Schematic presentation of dental features distinguishing Mustela putorius (1) from Mustela eversmanii (2). The features are indicated by red arrows, the numbers next to them correspond to the numbers of the characteristics described in the text.

The well-preserved polecat calvarium (FdN R 53), found with the right mandible (FdN R 54) in Coulet des Roches belongs to a very large adult animal (Figure 8) (Tables 2 and 3). The teeth are not yet very worn, and all the sutures are fully fused. The size of the skull (the individual is much larger when compared with the female from Aven des Planes (Vaucluse)), together with the strongly developed sagittal crest indicate that this specimen is a male. Additionally, the sex was confirmed by presence of the baculum, which is typical for the species morphology (Figure 9). The bone length is $40.6 \mathrm{~mm}$ and it has a strongly curved posterior end, while the anterior end is particularly broad, creating a robust bulge. 


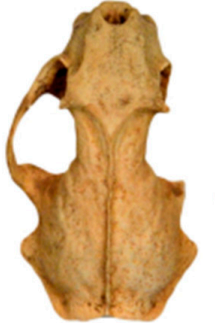

$1 \mathbf{a}$

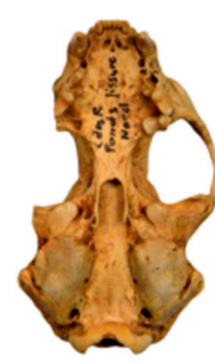

1b

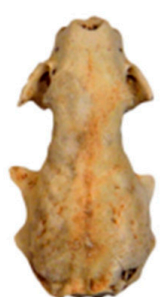

$\mathbf{2 a}$

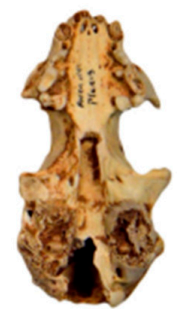

2b

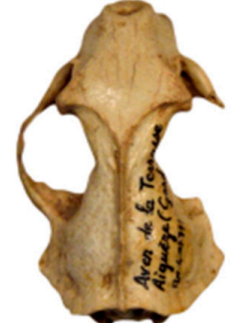

$\mathbf{3 a}$

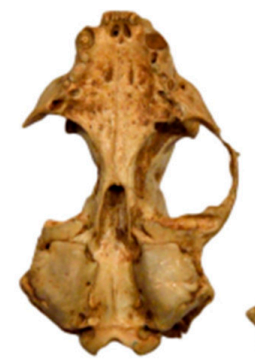

3b

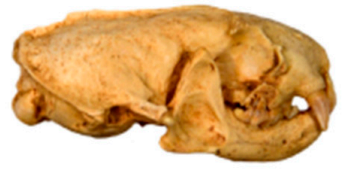

1c

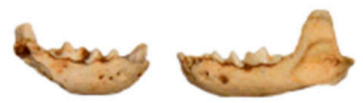

4

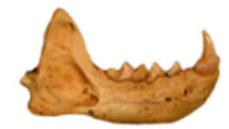

6

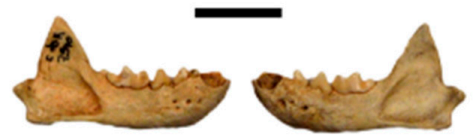

7

8

Figure 8. Cranial material of polecats. Mustela putorius: $0^{7}$ skull from Coulet des Roches FdN R 53 $(\mathbf{1 a}-\mathbf{c})$, q skull from Aven des Planes (2a,b), right (4) and left mandible (5) of $q$ from Aven des Planes, right $\sigma^{7}$ mandible from Coulet des Roches Fond nord (FdN R 53) (6), right R 7940 (7) and left $\sigma^{7}$ mandible R 3994 (8) from Coulet des Roches heap. Mustela eversmanii: $\sigma^{7}$ skull from Aven de la Terrasse (3a,b). Skulls showed in dorsal (a), ventral (b) and lateral (c) views, mandibles in buccal view. Scale bar $20 \mathrm{~mm}$. Photos: C. Triat.

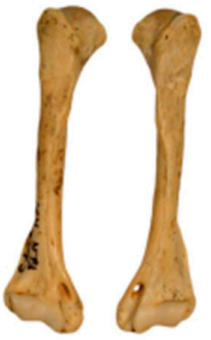

1

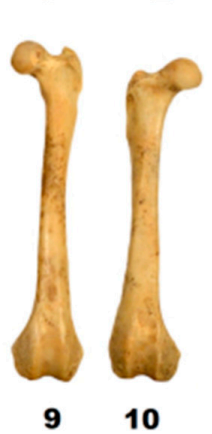

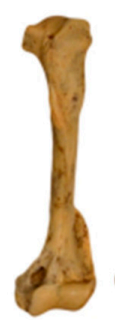

3

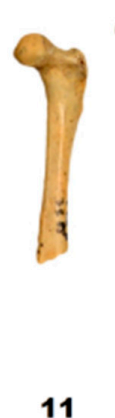

11

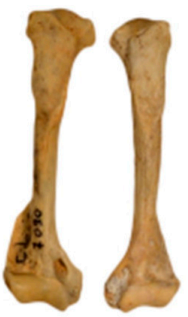

4
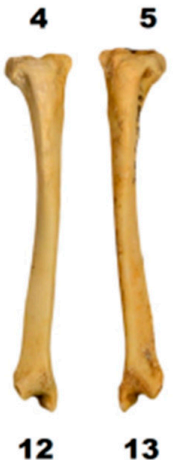
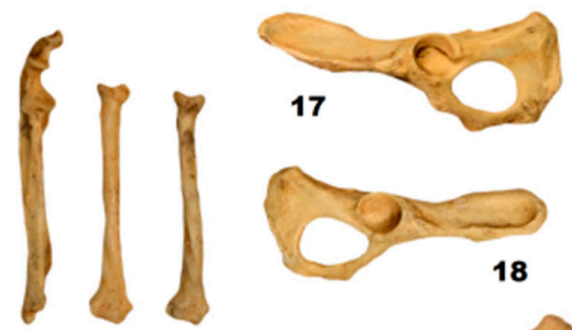

6
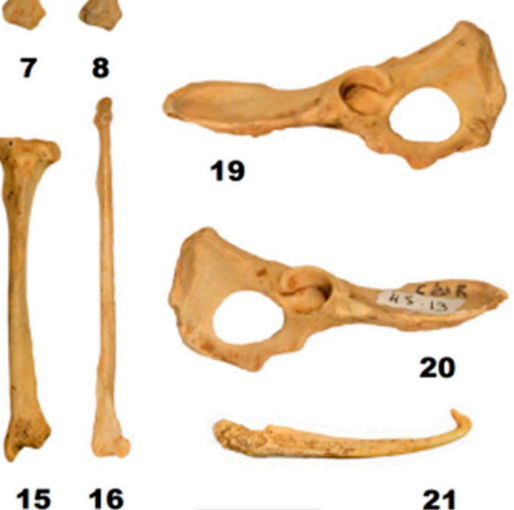

19
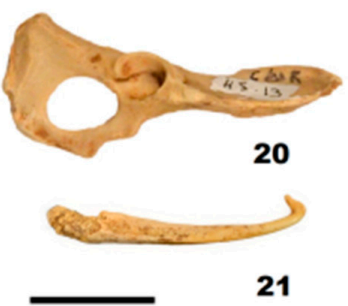

Figure 9. Postcranial material of Mustela putorius: right humerus (1); left humerus FdN R 35 (2); left humerus FdN R 36 (3); right humerus R 7096 (4); left humerus R 7090 (5); right ulna R 9326 (6); right radius G5 16-4 (7); right radius R 9325 (8); left femur G5 16-1 (9); right femur R 3288 (10); left femur R 9328 (11); right tibia G5 28-1 (12); left tibia G5 16-2 (13); right tibia R 8874 (14); left tibia R 8875 (15); left fibula M5 432-1 (16); left pelvis G5 18-1 (17); right pelvis R 8876 (18); left pelvis G5 18-1 (19); right pelvis H5 13 (20); and baculum (21). Scale bar 20 mm. Photos: C. Triat. 
Table 2. Dimensions of Mustela putorius and Mustela erminea skulls and upper teeth from Coulet des Roches (in mm). References of skull measurements in Figure 3. Abbreviations: P, premolar; M, molar; L, length; B, breadth; a, anterior; p, posterior; pr, protocone.

\begin{tabular}{|c|c|c|c|c|c|c|c|c|c|}
\hline & \multicolumn{2}{|c|}{ Mustela putorius } & \multicolumn{7}{|c|}{ Mustela erminea } \\
\hline & \multirow{2}{*}{$\begin{array}{c}\text { LGM } \\
\text { FdN }\end{array}$} & \multirow{3}{*}{$\begin{array}{c}\text { LG-GS 2a } \\
\text { Planes }\end{array}$} & \multicolumn{4}{|c|}{ LG-GS 2a } & \multicolumn{3}{|c|}{ LGM } \\
\hline & & & $\mathbf{R}$ & $\mathbf{R}$ & N4 & $\mathrm{J} 3$ & F5 & M5 & M6 \\
\hline & R53 & & 8092 & 7056 & 164 & 23 & 1 & 512 & 320 \\
\hline & $\sigma^{7}$ & q & q & $\sigma^{7}$ & $0^{x}$ & $\sigma^{7}$ & q & $\sigma^{7}$ & q \\
\hline 1 & - & & & & 45.4 & 43.3 & 42.0 & 46.1 & - \\
\hline 2 & 70.0 & - & - & - & 46.3 & 44.2 & 42.5 & 46.5 & - \\
\hline 3 & 66.3 & 59.2 & - & - & 43.1 & 41.2 & 39.5 & 43.8 & - \\
\hline 4 & - & - & - & - & 10.2 & 7.7 & - & 20.1 & - \\
\hline 5 & - & - & - & - & 27.2 & 28.6 & - & 28.2 & - \\
\hline 9 & - & - & 16.3 & - & 20.1 & 19.4 & 17.6 & 20.3 & - \\
\hline 10 & - & - & - & - & 16.3 & 15.7 & 14.9 & 12.3 & - \\
\hline 11 & 12.5 & 12.7 & 7.7 & - & 10.1 & 9.9 & 9.2 & 10.0 & - \\
\hline 12 & - & - & - & - & 26.4 & 26.0 & 25.3 & - & - \\
\hline 13 & 7.4 & 6.9 & 3.9 & - & 4.5 & 4.4 & 4.9 & 4.4 & - \\
\hline 14 & - & - & 9.5 & - & 12.8 & 11.2 & 10.1 & 11.8 & - \\
\hline 15 & - & - & - & - & 15.0 & 14.8 & 14.3 & - & - \\
\hline 16 & - & - & - & - & 15.9 & 15.9 & 14.5 & - & - \\
\hline 17 & - & - & 4.5 & - & 5.9 & 5.4 & 5.5 & 4.6 & - \\
\hline 18 & - & - & 10.9 & - & 13.9 & 13.3 & 12.0 & 12.5 & - \\
\hline 20 & 24.3 & 19.6 & 11.3 & 13.6 & 13.9 & 14.8 & 13.7 & 13.6 & 13.2 \\
\hline 21 & 15.3 & 14.7 & - & 12.6 & 11.5 & 12.9 & 12.4 & 9.2 & 12.7 \\
\hline 22 & - & - & - & 21.9 & 21.2 & 22.4 & 21.8 & 20.0 & 22.6 \\
\hline 23 & 39.1 & 31.4 & - & 20.9 & 22.0 & 21.8 & 21.1 & 22.3 & 22.5 \\
\hline 24 & 17.7 & 15.4 & - & 11.0 & 12.3 & 11.6 & 11.5 & 12.6 & 11.8 \\
\hline 25 & 9.7 & 6.7 & 4.1 & - & 4.4 & 5.1 & 4.1 & 4.8 & - \\
\hline 26 & 7.7 & 6.8 & 4.0 & - & 4.9 & 4.3 & 4.3 & 4.9 & - \\
\hline 27 & 10.0 & 8.7 & - & 6.2 & 6.9 & 6.6 & 6.8 & 6.5 & 5.1 \\
\hline 28 & 7.6 & - & - & 5.6 & 6.1 & & 5.3 & 6.0 & 6.3 \\
\hline 29 & - & - & - & 13.1 & 14.9 & 13.4 & 13.8 & 14.7 & 13.1 \\
\hline 30 & - & - & - & 7.5 & 8.4 & 8.6 & 7.6 & 8.1 & 8.1 \\
\hline 32 & 19.8 & 16.2 & - & - & - & - & - & - & - \\
\hline P3 L & 4.9 & 4.2 & - & - & - & - & - & - & - \\
\hline P3 B & 2.4 & 2.0 & - & - & - & - & - & - & - \\
\hline P4 L & 7.2 & 7.1 & 4.4 & - & 5.2 & 5.0 & 4.9 & 5.2 & - \\
\hline P4 L pr & - & & 1.1 & - & 0.9 & 1.1 & 1.4 & 3.0 & - \\
\hline $\mathrm{P} 4 \mathrm{Ba}$ & 4.4 & 4.1 & 2.1 & - & 2.6 & 2.6 & 2.3 & 2.7 & - \\
\hline P4 Bp & - & - & 1.4 & - & 1.8 & 1.6 & 1.6 & 1.9 & - \\
\hline M1 L & - & - & - & - & 4.2 & 4.3 & 3.9 & 4.5 & - \\
\hline M1 B1 & - & - & - & - & 1.6 & 2.0 & 1.8 & 2.0 & - \\
\hline M1 B2 & - & - & - & - & 1.7 & 1.7 & 1.7 & 1.5 & - \\
\hline M1 B3 & - & - & - & - & 2.0 & 2.0 & 2.2 & 2.3 & - \\
\hline
\end{tabular}


Table 3. Dimensions of Mustela putorius and Mustela erminea mandible from Coulet des Roches (in mm). References of mandible measurements in Figures 4 and 5. Abbreviations: L, left; R, right; p, premolar; $\mathrm{m}$, molar; L, length; B, breadth; a, anterior; p, posterior; tri, trigonid; tal, talonid.

\begin{tabular}{|c|c|c|c|c|c|c|c|c|c|c|c|c|c|c|c|}
\hline & \multicolumn{6}{|c|}{ Mustela putorius } & \multicolumn{9}{|c|}{ Mustela erminea } \\
\hline & \multicolumn{4}{|c|}{ Coulet: LGM } & \multicolumn{2}{|c|}{ LG-GS 2a } & \multicolumn{6}{|c|}{ LG-GS 2a } & \multicolumn{3}{|c|}{ LGM } \\
\hline & $\mathbf{R}$ & $\mathbf{R}$ & $\mathbf{R}$ & $\mathbf{R}$ & Plar & & $\mathbf{R}$ & $\mathbf{R}$ & $\mathbf{R}$ & $\mathbf{R}$ & M4 & M3-4 & M4 & F4-G4 & F5 G5 \\
\hline & 3994 & 7940 & 3995 & 54 & & & 3037 & 3058 & 8871 & 9309 & 195 & 1.1 & $988-2$ & R 1-6 & R 1-4 \\
\hline & $0^{\pi}$ & $0^{\pi}$ & $\sigma^{7}$ & $\sigma^{x}$ & $\varphi(\mathbf{L})$ & $\varphi(\mathbf{R})$ & q & 우 & $\sigma^{x}$ & $0^{\pi}$ & $0^{x}$ & $\sigma^{7}$ & q & q & ㅇ \\
\hline 1 & 44.4 & 44.8 & - & 41.7 & 36.0 & - & - & 21.4 & 25.7 & 18.7 & 25.8 & 20.4 & 18.6 & - & - \\
\hline 2 & 43.5 & 44.6 & - & 41.6 & 35.6 & - & - & 20.1 & 23.6 & 17.4 & 23.5 & 19.0 & 17.1 & - & - \\
\hline 3 & 36.3 & 37.1 & - & 35.0 & 31.5 & - & - & 14.0 & 16.2 & 11.8 & 16.0 & 13.1 & 12.7 & - & - \\
\hline 4 & 21.5 & 21.5 & - & 20.0 & 18.7 & 18.4 & 11.2 & 13.4 & 14.9 & 11.8 & 15.5 & 13.1 & 12.5 & - & - \\
\hline 5 & 20.2 & 20.5 & - & 19.0 & 18.4 & 17.8 & 4.9 & 11.7 & 12.1 & 9.7 & 12.2 & 10.8 & 10.2 & - & - \\
\hline 6 & 9.9 & 10.4 & 9.3 & 9.3 & 9.1 & 8.2 & 6.0 & 4.5 & 5.1 & 4.4 & 6.7 & 5.1 & 4.7 & - & - \\
\hline 7 & 10.8 & 10.8 & 10.6 & 10.7 & 10.2 & 9.8 & 1.7 & 6.6 & 7.0 & 5.9 & 6.6 & 5.6 & 5.9 & - & - \\
\hline 8 & 5.1 & 5.0 & 3.5 & 3.4 & 4.4 & 3.3 & - & 1.9 & 2.4 & 2.0 & 2.6 & 2.1 & 1.8 & - & - \\
\hline 10 & - & - & - & - & - & - & - & 10.2 & 13.0 & & 12.7 & 9.6 & 8.0 & - & - \\
\hline 12 & 10.4 & 10.0 & 10.2 & 10.7 & 7.7 & 8.1 & - & 4.9 & 4.5 & 3.3 & 5.1 & 3.6 & 3.4 & - & - \\
\hline 14 & 9.9 & 10.0 & 10.2 & 10.7 & 8.1 & - & 3.7 & 4.2 & 5.6 & 3.9 & 4.5 & 3.9 & 2.7 & - & - \\
\hline 17 & 13.0 & 13.6 & - & 13.1 & 11.4 & - & - & 4.4 & - & 4.7 & 6.7 & 3.9 & 4.5 & - & - \\
\hline c1 L & - & 5.3 & - & 4.8 & 3.5 & 3.9 & - & - & - & - & - & - & - & - & - \\
\hline c1 B & - & 3.9 & - & 3.7 & 2.8 & 3.3 & - & - & - & - & - & - & - & - & - \\
\hline p2 L & - & 2.7 & - & - & - & 2.4 & - & - & - & - & - & - & - & - & - \\
\hline p2 B & - & 2.4 & - & - & - & 1.4 & - & - & - & - & - & - & - & - & - \\
\hline p3 L & 3.6 & 3.5 & 4.0 & - & 3.7 & 4.0 & - & - & - & - & - & - & - & - & - \\
\hline p3 B & 2.4 & 2.1 & 2.4 & 2.1 & 1.9 & 1.8 & - & - & - & - & - & - & - & - & - \\
\hline $\mathrm{p} 4 \mathrm{~L}$ & 4.7 & 4.6 & 4.5 & 4.5 & 4.2 & 4.4 & - & - & 2.6 & 3.1 & - & 3.3 & - & - & 2.8 \\
\hline p4 Ba & - & - & - & & - & - & - & - & 1.1 & 1.4 & - & 1.2 & - & - & 1.1 \\
\hline p4 Bp & 2.6 & 2.6 & 2.6 & 2.4 & 2.1 & 2.1 & - & - & 1.4 & 1.7 & - & 1.6 & - & - & 1.3 \\
\hline $\mathrm{m} 1 \mathrm{~L}$ & 9.0 & 8.6 & 9.0 & 8.9 & 8.2 & 8.1 & - & 5.2 & 5.5 & 5.7 & 4.6 & 5.6 & 5.0 & 5.3 & - \\
\hline $\mathrm{m} 1 \mathrm{~L}$ tri & 6.8 & 6.5 & 6.7 & 6.6 & 5.7 & 5.9 & - & 3.7 & 4.3 & 4.3 & 3.4 & 4.4 & 3.7 & 3.5 & - \\
\hline $\mathrm{m} 1 \mathrm{~L}$ tal & - & - & - & - & - & - & - & 1.3 & 1.6 & 1.6 & 1.6 & 1.7 & 1.2 & 1.5 & - \\
\hline $\mathrm{m} 1 \mathrm{~B}$ tri & 3.8 & 3.5 & 3.8 & 3.6 & 3.1 & 3.2 & - & 1.8 & 1.9 & 2.1 & 2.1 & 2.4 & 1.8 & 1.7 & - \\
\hline m1 B tal & 2.6 & 2.5 & 2.7 & 2.6 & 2.3 & 2.4 & - & 1.5 & 1.6 & 1.7 & 1.4 & 1.9 & 1.4 & 1.4 & - \\
\hline $\mathrm{m} 2 \mathrm{~L}$ & - & 2.2 & - & - & - & - & - & - & - & 1.2 & - & 1.3 & - & - & - \\
\hline $\mathrm{m} 2 \mathrm{~B}$ & - & 1.7 & - & - & - & - & - & - & - & 1.2 & - & 0.9 & - & - & - \\
\hline
\end{tabular}

The analyzed specimen can be classified as M. putorius, on the basis of its morphology. Although the individual from Coulet des Roches is almost as large as the M. eversmanii male from Aven de la Terrasse (Gard) [38], overall the skull is less robust (Figure 10). The elongated and cylindrical shape of the cranial section between the frontal and postorbital processes, with a narrower rostrum, less expanded zygomatic arches, with less developed, although still well-defined muscle attachments and sagittal crest, oval nasal aperture, with broad, V-shaped palatal posterior part, proportionally smaller orbits and relatively large M1, clearly indicate M. putorius (Figure 11). In addition, joined to the calvarium, the right mandible comprises many of the morphometric features characteristic of M. putorius. Apart from the large size and robustly built body, the rounded anterior margin of quite deep masseter fossa does not reach the $\mathrm{m} 1 / \mathrm{m} 2$ border, the margins of the upper ramus create a relatively open angle, and the alveolar length of the $\mathrm{m} 2$ appears to be reduced, but is still larger than in M. eversmanii. 


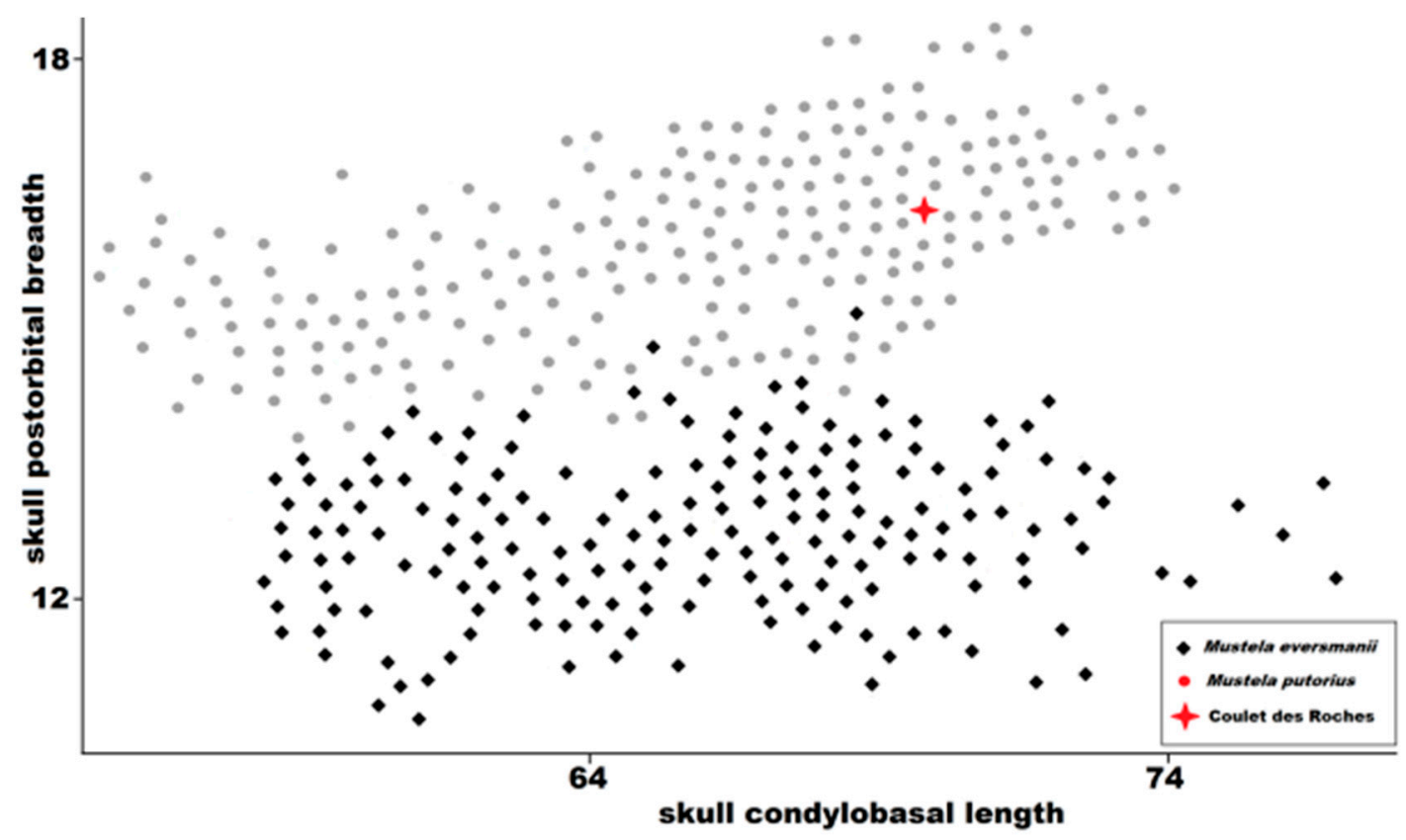

Figure 10. Postorbital skull breadth plotted against condylobasal length in Mustela eversmanii and Mustela putorius. Data from Marciszak [21], Koby [27], Zapfe [31], Sickenberg [33], Hugueney [35], and Crégut-Bonnoure and Guérin [38].

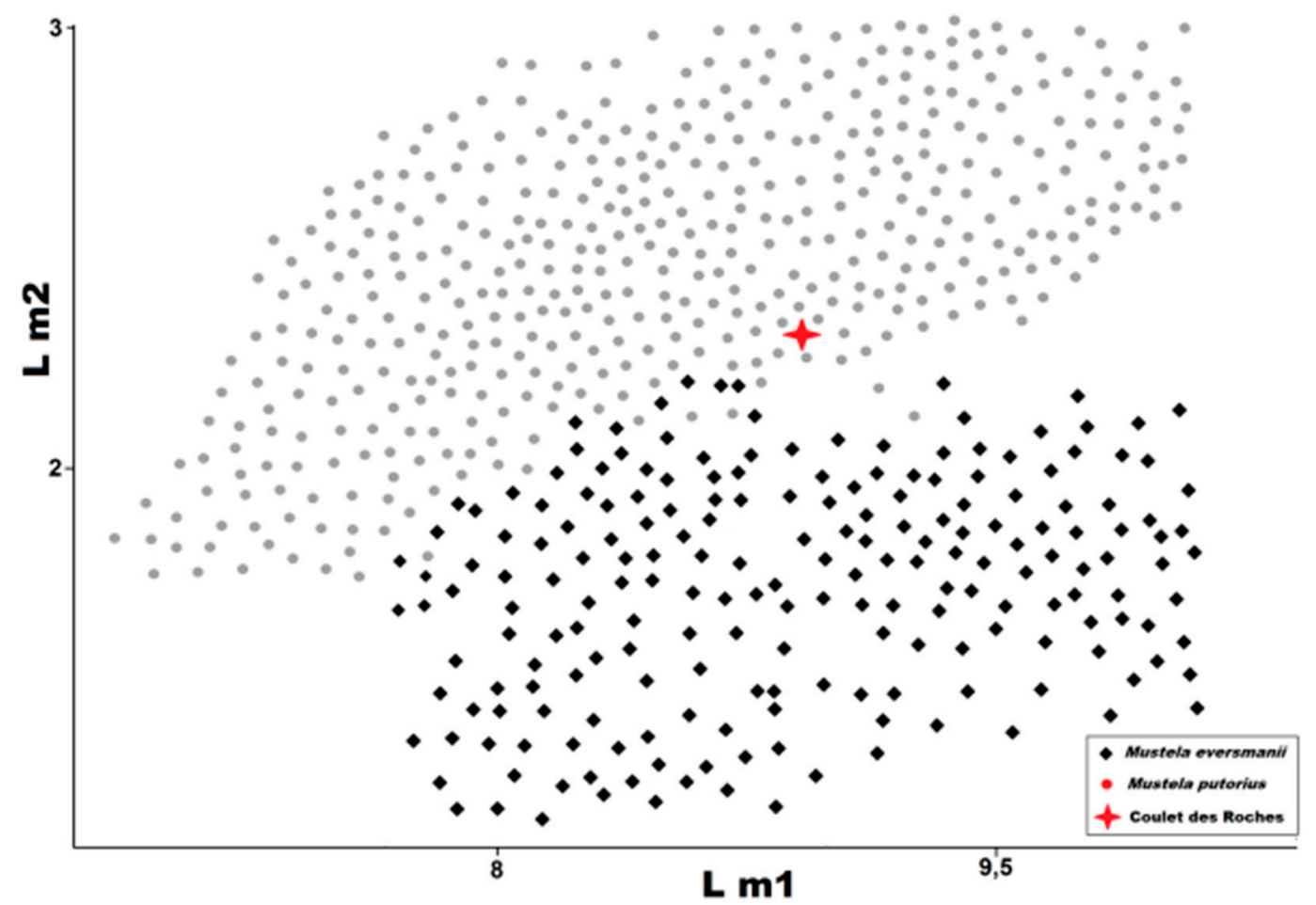

Figure 11. Length of $\mathrm{m} 1$ (L m1) plotted against length of $\mathrm{m} 2$ (L m2) in Mustela eversmanii and Mustela putorius. Data from Marciszak [21], Koby [27], Zapfe [31], Sickenberg [33], Hugueney [35], and Crégut-Bonnoure and Guérin [38].

At many other sites, such as Coulet des Roches, numerous and often well-preserved postcranial polecat remains have been found (Figure 9) (Tables 4-8). However, despite some previous attempts $[44,45]$ to find differences in the morphology and proportions of long bones, no reliable metric or morphological features distinguish the two polecat species. Some authors found minor 
differences, such as humerus morphology [27,42,45]. However, these studies were usually based on a relatively small quantity of material, which does not reflect the high variation in the size of both species. On average, the postcranial material of M. eversmanii, especially long bones and metapodials, is larger and more robust (sex should be taken into consideration). However, the similarity in the morphology, proportions and dimensions of both polecats is so strong that postcranial material is usually classified as a large member of Mustela sp. Reliable taxonomical classification can only be attempted when these remains are clearly associated with cranial material.

Table 4. Dimensions of mustelid humeri from Coulet des Roches (in mm). References of measurements: 1 , greatest length; 2 , proximal depth; 3 , proximal breadth; 4 , midshaft diaphyseal depth; 5 , diaphyseal midshaft breadth; 6 , distal depth; 7 , distal breadth; 8 , trochlear breadth.

\begin{tabular}{|c|c|c|c|c|c|c|c|c|c|c|}
\hline Species & $\begin{array}{c}\text { Collection } \\
\text { No. }\end{array}$ & Sex & 1 & 2 & 3 & 4 & 5 & 6 & 7 & 8 \\
\hline \multicolumn{11}{|c|}{ LGM } \\
\hline Mustela putorius & FdN R 36 & $\sigma^{7}$ & 51.9 & 10.5 & 11.5 & 5.8 & 4.6 & 6.9 & 13.7 & 9.5 \\
\hline Mustela putorius & FdN R 35 & $\sigma^{7}$ & 51.6 & 10.3 & 11.4 & 5.7 & 5.7 & 6.8 & 13.5 & 9.4 \\
\hline Mustela putorius & R 7090 & $\sigma^{x}$ & 47.8 & 11.0 & 11.6 & 4.8 & 4.8 & 7.7 & 13.4 & 10.7 \\
\hline Mustela putorius & R 7096 & $0^{x}$ & 46.6 & 11.5 & 11.4 & 4.6 & 4.6 & 7.3 & 13.5 & 11.4 \\
\hline \multicolumn{11}{|c|}{ LG-GS 2a } \\
\hline Mustela erminea & R 3043 & $0^{x}$ & 34.4 & 5.8 & 5.8 & 2.6 & 2.4 & 3.6 & 6.4 & 5.5 \\
\hline Mustela erminea & R 3044 & $\sigma^{\pi} / p$ & 33.2 & 6.3 & 6.6 & 2.8 & 2.5 & 3.7 & 6.9 & 5.1 \\
\hline Mustela erminea & R 9295 & q & 26.7 & 5.6 & 5.4 & 2.4 & 2.3 & 3.2 & 5.8 & 4.4 \\
\hline Mustela erminea & M4 181-20 & $0^{7}$ & 35.4 & 6.2 & 6.6 & 2.7 & 2.2 & 3.7 & 7.0 & 5.9 \\
\hline Mustela erminea & G4 5-37 & $\sigma^{7}$ & - & - & - & 2.7 & 2.4 & 3.8 & 7.0 & 5.4 \\
\hline \multicolumn{11}{|c|}{ LGM } \\
\hline Mustela erminea & M4 R 449 (L) & $\sigma^{7}$ & 22.3 & 6.2 & 4.1 & 1.7 & 1.7 & 2.8 & 4.6 & 4.2 \\
\hline Mustela erminea & M4 R 449 (R) & $0^{7}$ & - & - & - & 1.9 & 1.7 & 2.6 & 4.8 & 4.5 \\
\hline \multicolumn{11}{|c|}{ GI 3} \\
\hline Mustela erminea & G4 26-26 & $\sigma^{7}$ & 31.3 & 6.4 & 5.9 & 2.4 & 2.5 & 3.5 & 6.1 & 4.9 \\
\hline \multicolumn{11}{|c|}{ LG-GS 2a } \\
\hline Mustela nivalis & R 3016 & $0^{x}$ & - & - & - & 2.0 & 2.0 & 3.3 & 5.4 & 4.1 \\
\hline Mustela nivalis & R 3042 & q & 21.7 & 4.1 & 4.6 & 1.9 & 1.5 & 2.6 & 5.1 & 4.1 \\
\hline \multicolumn{11}{|l|}{ LGM } \\
\hline Mustela nivalis & M4 214 & $\sigma^{7}$ & 17.0 & 3.4 & 3.6 & 1.3 & 1.1 & 2.2 & 4.1 & 3.5 \\
\hline Mustela nivalis & N3-N4 R 30 & $\sigma^{7}$ & 20.4 & 4.2 & 4.2 & 1.6 & 1.5 & 2.4 & 4.6 & 3.4 \\
\hline Mustela nivalis & M4 217 & $\sigma^{x}$ & 16.8 & 3.3 & 3.7 & 1.4 & 1.1 & 2.3 & 4.2 & 3.3 \\
\hline Mustela nivalis & N5-N6 R 115 & q & 20.0 & 4.0 & 4.1 & 1.8 & 1.4 & 2.5 & 4.5 & 3.9 \\
\hline Mustela nivalis & H5 R 2-5 & $0^{7}$ & 23.7 & 4.3 & 4.5 & 1.9 & 1.6 & 3.0 & 5.0 & 3.8 \\
\hline Mustela nivalis & H4 7-285 & $0^{x}$ & 23.8 & 4.3 & 4.4 & 1.8 & 1.6 & 3.0 & 5.0 & 4.2 \\
\hline Mustela nivalis & G4 6-13 & q & 19.7 & 3.6 & 4.1 & 1.5 & 1.5 & 2.6 & 4.5 & 4.1 \\
\hline Mustela nivalis & G5 7-55 & q & - & - & - & - & - & 2.7 & 4.4 & 3.8 \\
\hline Mustela nivalis & G5 7-56 & $0^{7}$ & - & - & - & 1.9 & 1.4 & 2.8 & 4.3 & 3.8 \\
\hline Mustela nivalis & $\begin{array}{c}\text { M3-M4 R } \\
71-2\end{array}$ & $0^{7}$ & - & - & - & - & - & 2.8 & 4.7 & 3.0 \\
\hline \multicolumn{11}{|c|}{ GI 3} \\
\hline Mustela nivalis & L6 R 8-3 & $\sigma^{x}$ & 25.4 & 4.5 & 4.4 & 2.0 & 2.1 & 2.8 & 4.9 & 3.6 \\
\hline Mustela nivalis & L6 R 8-3 & $\sigma^{7}$ & 25.4 & 4.4 & 4.4 & 1.9 & 2.0 & 3.2 & 5.0 & 4.2 \\
\hline Mustela nivalis & K5 R 76-3 & $0^{7}$ & 28.0 & - & - & 2.5 & 2.3 & 3.1 & 5.5 & 4.7 \\
\hline \multicolumn{11}{|c|}{ GI 10-11 } \\
\hline Mustela nivalis & N5 408-6 & $0^{x}$ & 24.3 & 4.3 & 4.4 & 2.0 & 1.9 & 2.8 & 5.3 & 3.9 \\
\hline
\end{tabular}


Table 5. Dimensions of mustelid radiuses from Coulet des Roches (in $\mathrm{mm}$ ). References of measurements: 1 , greatest length; 2 , proximal depth; 3 , proximal breadth; 4 , midshaft diaphyseal depth; 5 , diaphyseal midshaft breadth; 6 , distal depth; 7 , distal breadth; 8 , trochlear breadth.

\begin{tabular}{|c|c|c|c|c|c|c|c|c|c|}
\hline Species & Collection No. & Sex & 1 & 2 & 3 & 4 & 5 & 6 & 7 \\
\hline \multicolumn{10}{|c|}{ LGM } \\
\hline Mustela putorius & R 9327 & $\sigma^{7}$ & 37.2 & 4.4 & 5.9 & 2.7 & 3.5 & 7.4 & 5.5 \\
\hline Mustela putorius & G5 14-4 & $\sigma^{7}$ & 38.3 & 4.1 & 5.8 & 2.7 & 3.7 & 7.8 & 5.4 \\
\hline \multicolumn{10}{|c|}{ LG-GS 2a } \\
\hline Mustela erminea & R 3048 & $\sigma^{7}$ & 23.5 & 1.8 & 2.8 & 1.6 & 1.9 & 3.2 & 2.5 \\
\hline Mustela erminea & M4 183 & $\sigma^{7}$ & 25.2 & 2.2 & 3.4 & 1.5 & 1.5 & 2.9 & 4.1 \\
\hline \multicolumn{10}{|c|}{ LGM } \\
\hline Mustela erminea & F5 R 3-8 & 우 & 21.2 & 1.9 & 3.0 & 1.4 & 2.0 & 3.7 & 3.7 \\
\hline Mustela erminea & F5-G 51 & 우 & 21.4 & 2.1 & 3.0 & 1.5 & 1.6 & 2.5 & 3.7 \\
\hline Mustela erminea & F5 R 4-11 & 우 & 22.7 & 1.8 & 3.1 & 1.6 & 1.8 & 2.6 & 3.8 \\
\hline \multicolumn{10}{|c|}{ LGM } \\
\hline Mustela nivalis & M3-M4 R 1-4 & $\sigma^{7}$ & - & 1.5 & 2.2 & 0.9 & 1.3 & - & - \\
\hline Mustela nivalis & M4 217 & $0^{x}$ & 11.3 & 1.2 & 1.9 & 0.6 & 0.9 & 1.6 & 2.4 \\
\hline \multicolumn{10}{|c|}{ GI 3} \\
\hline Mustela nivalis & K5 R 76-6 & $\sigma^{7}$ & 20.0 & 1.8 & 2.8 & 1.5 & 1.6 & - & - \\
\hline Mustela nivalis & L5-L6 R 7-3 & $\sigma^{7}$ & 21.0 & 2.0 & 2.9 & 1.4 & 1.6 & - & - \\
\hline
\end{tabular}

Table 6. Dimensions of mustelid ulnae from Coulet des Roches (in $\mathrm{mm}$ ). References of measurements: 1 , greatest length; 2 , proximal depth; 3 , proximal breadth; 4, midshaft diaphyseal depth; 5 , diaphyseal midshaft breadth; 6 , distal depth; 7 , distal breadth; 8 , trochlear breadth.

\begin{tabular}{|c|c|c|c|c|c|c|c|c|c|}
\hline Species & Collection No. & Sex & 1 & 2 & 3 & 4 & 5 & 6 & 7 \\
\hline \multicolumn{10}{|c|}{ LGM } \\
\hline Mustela putorius & R 9326 & $\sigma^{x}$ & 47.4 & 8.3 & 4.9 & 3.8 & 3.1 & 6.4 & 3.8 \\
\hline \multicolumn{10}{|c|}{ LGM } \\
\hline Mustela erminea & M4 R 449 & $\sigma^{7}$ & - & 2.9 & 2.4 & - & - & - & - \\
\hline Mustela erminea & F4 R 1-8 & q & 27.2 & 3.5 & 3.0 & 2.3 & 1.4 & 2.2 & 2.1 \\
\hline Mustela erminea & F5 R 1-3 & q & & & & 2.4 & 1.5 & 3.2 & 2.0 \\
\hline \multicolumn{10}{|c|}{ GI 3} \\
\hline Mustela erminea & G4 26-27 & 우 & 26.9 & 3.6 & 3.1 & 2.1 & 1.5 & 3.2 & 2.1 \\
\hline \multicolumn{10}{|c|}{ LG-GS 2a } \\
\hline Mustela nivalis & R 3045 & $\sigma^{7}$ & 19.2 & 4.1 & 3.3 & 2.4 & 1.4 & 3.4 & 1.9 \\
\hline Mustela nivalis & R 3046 & $0^{x}$ & 21.0 & 3.3 & 3.1 & 1.8 & 1.3 & 2.1 & 1.6 \\
\hline Mustela nivalis & M3 53-57 & $0^{7}$ & 19.1 & 2.8 & 2.4 & 1.5 & 0.8 & 2.0 & 1.2 \\
\hline \multicolumn{10}{|c|}{ LGM } \\
\hline Mustela nivalis & M3-M4 R 1-3 & $\sigma^{7}$ & 18.1 & 2.8 & 2.4 & 1.5 & 0.7 & 1.8 & 1.5 \\
\hline Mustela nivalis & M4 217 & $0^{7}$ & 15.2 & 2.3 & 1.6 & 0.8 & 0.8 & 1.7 & 1.0 \\
\hline Mustela nivalis & M5 R 27 & q & - & 2.8 & 2.5 & 1.8 & 0.8 & - & - \\
\hline Mustela nivalis & L5 R 21-4 & $0^{7}$ & - & 3.0 & 2.3 & - & - & - & - \\
\hline \multicolumn{10}{|c|}{ GI 3} \\
\hline Mustela nivalis & K5 R 76-5 & $\sigma^{7}$ & 25.0 & 3.7 & 3.1 & 1.8 & 1.3 & - & - \\
\hline
\end{tabular}


Table 7. Dimensions of mustelid femurs from Coulet des Roches (in mm). References of measurements: 1 , greatest length; 2 , proximal depth; 3 , proximal breadth; 4 , midshaft diaphyseal depth; 5 , diaphyseal midshaft breadth; 6, distal depth; 7, distal breadth; 8: trochlear breadth.

\begin{tabular}{|c|c|c|c|c|c|c|c|c|c|}
\hline Species & Collection No. & Sex & 1 & 2 & 3 & 4 & 5 & 6 & 7 \\
\hline \multicolumn{10}{|c|}{ LGM } \\
\hline Mustela putorius & R 9328 & $\sigma^{7}$ & 54.6 & 13.1 & 6.8 & 3.9 & 4.9 & 9.5 & 12.6 \\
\hline Mustela putorius & R 3288 & $\sigma^{7}$ & - & 13.5 & 6.8 & 4.0 & 5.4 & - & - \\
\hline Mustela putorius & G5 16-1 & $\sigma^{7}$ & 56.5 & 12.6 & 6.5 & 4.7 & 4.7 & 9.1 & 12.4 \\
\hline \multicolumn{10}{|c|}{ Holocene-Atlantic } \\
\hline Mustela erminea & J3-K3 R 11 & $\sigma^{7}$ & 27.9 & 5.2 & 2.9 & 2.1 & 2.2 & 4.2 & 4.9 \\
\hline Mustela erminea & J3-K3 R 11 & $0^{7}$ & 27.7 & 5.2 & 2.7 & 2.0 & 2.0 & 4.4 & 4.9 \\
\hline \multicolumn{10}{|c|}{ LG-GS 2a } \\
\hline Mustela erminea & R 3057 & 우 & 31.1 & 6.4 & 3.1 & 2.3 & 2.4 & 4.9 & 5.6 \\
\hline Mustela erminea & R 9297 & $\sigma^{7}$ & 35.5 & 6.7 & 3.5 & 2.7 & 2.8 & 5.7 & 6.3 \\
\hline \multicolumn{10}{|c|}{ LGM } \\
\hline Mustela erminea & F4 R 4-4 & q & 31.9 & 6.2 & 2.9 & 2.6 & 2.4 & 4.5 & 5.9 \\
\hline Mustela erminea & F4 R 4-5 & 우 & 32.1 & 6.1 & 3.1 & 2.5 & 2.7 & 4.6 & 5.9 \\
\hline \multicolumn{10}{|c|}{ LG-GS 2a } \\
\hline Mustela nivalis & R 3049 & $\sigma^{7}$ & 23.9 & 5.6 & 2.9 & 2.0 & 2.2 & 4.2 & 4.9 \\
\hline Mustela nivalis & R 3056 & $\sigma^{x}$ & 25.6 & 5.8 & 3.0 & 2.0 & 2.2 & 4.1 & 5.0 \\
\hline Mustela nivalis & M3 53-55 & $0^{7}$ & 21.8 & 4.8 & 2.5 & 1.9 & 1.9 & 3.9 & 4.8 \\
\hline \multicolumn{10}{|c|}{ LGM } \\
\hline Mustela nivalis & M4 217 & $0^{7}$ & 16.3 & 3.3 & 2.0 & 1.2 & 1.2 & 3.0 & 3.5 \\
\hline Mustela nivalis & M4 217 & $0^{x}$ & 16.3 & 3.4 & 1.9 & 1.3 & 1.2 & 3.1 & 3.5 \\
\hline Mustela nivalis & M3-M4 R 56 & $0^{2}$ & 19.8 & 4.5 & 2.6 & 1.5 & 1.8 & 3.7 & 4.4 \\
\hline Mustela nivalis & M4 R 329 & $\sigma^{7}$ & 20.2 & 5.1 & 2.7 & 1.7 & 1.9 & 3.7 & 4.4 \\
\hline \multicolumn{10}{|c|}{ GI 3} \\
\hline Mustela nivalis & L6 R 10-2 & $0^{7}$ & 27.0 & 5.3 & 2.7 & 2.0 & 2.0 & 4.1 & 4.8 \\
\hline Mustela nivalis & K5 R 76-7 & $0^{x}$ & 28.0 & - & - & 2.3 & 2.7 & - & - \\
\hline
\end{tabular}

Table 8. Dimensions of mustelid tibias from Coulet des Roches (in $\mathrm{mm}$ ). References of measurements: 1 , greatest length; 2 , proximal depth; 3 , proximal breadth; 4 , midshaft diaphyseal depth; 5 , diaphyseal midshaft breadth; 6, distal depth; 7, distal breadth; 8: trochlear breadth.

\begin{tabular}{|c|c|c|c|c|c|c|c|c|c|}
\hline Species & Collection No. & Sex & 1 & 2 & 3 & 4 & 5 & 6 & 7 \\
\hline \multicolumn{10}{|c|}{ LGM } \\
\hline Mustela putorius & R 8874 & $\sigma^{x}$ & 54.4 & 9.7 & 11.7 & 5.2 & 3.3 & 6.8 & 8.7 \\
\hline Mustela putorius & R 8875 & $\sigma^{7}$ & 54.0 & 9.8 & 11.8 & 5.1 & 3.4 & 6.4 & 8.6 \\
\hline Mustela putorius & G5 16-2 & $\sigma^{7}$ & 60.7 & 9.8 & 11.8 & 5.1 & 4.2 & 6.8 & 8.1 \\
\hline Mustela putorius & G5 28-1 & $\sigma^{7}$ & 60.0 & 9.7 & 11.6 & 4.5 & 4.2 & 6.7 & 7.8 \\
\hline \multicolumn{10}{|c|}{ LG-GS 2a } \\
\hline Mustela erminea & Planes 2017 & $\sigma^{7}$ & 42.3 & 5.9 & 7.0 & 3.1 & 2.4 & 3.8 & 4.9 \\
\hline \multicolumn{10}{|c|}{ LG-GS 2a } \\
\hline Mustela erminea & R 3051 & 우 & 34.4 & 5.0 & 5.7 & 2.2 & 1.9 & 3.0 & 4.0 \\
\hline Mustela erminea & M4 191-7 & $\sigma^{7}$ & 40.3 & 5.7 & 6.2 & 2.5 & 2.1 & 3.6 & 4.7 \\
\hline \multicolumn{10}{|c|}{ LGM } \\
\hline Mustela erminea & F4 R1-5 & 우 & 34.3 & 4.9 & 5.8 & 2.4 & 2.2 & 3.1 & 4.2 \\
\hline Mustela erminea & F4 R1-5 & q & 34.4 & 4.6 & 5.8 & 2.3 & 2.1 & 3.2 & 4.1 \\
\hline
\end{tabular}


Table 8. Cont.

\begin{tabular}{|c|c|c|c|c|c|c|c|c|c|}
\hline \multicolumn{10}{|c|}{ LG-GS 2a } \\
\hline Mustela nivalis & R 3050 & $0^{x}$ & 28.3 & 4.7 & 5.2 & 2.1 & 1.8 & 3.1 & 3.7 \\
\hline Mustela nivalis & R 3052 & $\sigma^{7}$ & 27.8 & 4.3 & 4.9 & 2.0 & 1.6 & 2.6 & 3.4 \\
\hline Mustela nivalis & R 3053 & q & 22.3 & 3.4 & 4.5 & 1.5 & 1.0 & 2.5 & 2.9 \\
\hline Mustela nivalis & R 3054 & $0^{7}$ & 24.5 & 3.8 & 4.6 & 1.9 & 1.6 & 2.4 & 3.1 \\
\hline Mustela nivalis & R 3055 & $0^{x}$ & 26.3 & 3.8 & 3.8 & 1.6 & 1.5 & 2.6 & 3.4 \\
\hline \multicolumn{10}{|c|}{ LGM } \\
\hline Mustela nivalis & M3 53-56 & $\sigma^{7}$ & & 3.9 & 4.5 & 1.6 & 1.4 & & \\
\hline Mustela nivalis & M4 217 & $\sigma^{7}$ & 17.6 & 2.8 & 3.5 & 1.2 & 0.9 & 1.7 & 2.5 \\
\hline Mustela nivalis & M4 217 & $\sigma^{7}$ & 17.7 & 2.8 & 3.6 & 1.2 & 0.9 & 1.7 & 2.5 \\
\hline Mustela nivalis & M3-M4 R 46 & $0^{x}$ & 21.2 & 3.8 & 4.2 & 1.6 & 1.2 & 2.1 & 3.0 \\
\hline Mustela nivalis & M3-M4 R 46 & $\sigma^{7}$ & 21.4 & 3.7 & 4.2 & 1.4 & 1.3 & 2.1 & 3.1 \\
\hline Mustela nivalis & N5-N6 R 175 & q & 21.0 & 3.7 & 3.9 & 1.2 & 1.1 & 2.1 & 2.8 \\
\hline Mustela nivalis & L5 53-2 & $0^{7}$ & - & - & - & 1.9 & 1.9 & - & - \\
\hline Mustela nivalis & G5 10-2 & $0^{7}$ & 26.4 & 4.0 & 4.5 & 1.5 & 1.4 & 2.3 & 3.4 \\
\hline Mustela nivalis & G4 R 15-6 & $0^{7}$ & 17.9 & 2.7 & 3.4 & 1.4 & 1.0 & 2.1 & 2.2 \\
\hline \multicolumn{10}{|c|}{ GI 10-11 } \\
\hline Mustela nivalis & M4-N4 R 46-2 & $\sigma^{7}$ & - & - & - & 1.7 & 1.5 & - & - \\
\hline
\end{tabular}

4.1.2. Mustela erminea Linnaeus, 1758 and Mustela nivalis Linnaeus, 1766

Species diagnosis

Like for many other mustelid species, M. erminea and M. nivalis are morphologically very similar and distinguishing them can be a risky task, particularly on the basis of isolated remains. On average, the stoat is larger than the smallest weasel, however, both mustelids are extremely metrically variable, due to geographical variation and marked sexual dimorphism. In many areas, large males from the smallest weasel are comparable or even larger than small stoat females. After detailed analysis, the following features were considered to be reliable criteria for distinguishing both mustelids (M. erminea compared to M. nivalis) (for indexes the mean was given) (Figures 12-16) (Tables 3-10) [32,35,36,42,43,46-58]:

1. Overall larger size: condylobasal skull length in M. erminea $\mathrm{o}^{\mathrm{T}}$ is $49.85 \mathrm{~mm}, 42.00 \mathrm{~mm}$ in M. erminea , $40.67 \mathrm{~mm}$ in $M$. nivalis $\sigma^{7}$ and $32.88 \mathrm{~mm}$ in $M$. nivalis ; total mandible length in M. erminea $\sigma^{7}$ is $29.02 \mathrm{~mm}, 22.23 \mathrm{~mm}$ in M. erminea $9,21.94 \mathrm{~mm}$ in M. nivalis $0^{7}$ and $16.52 \mathrm{~mm}$ in M. nivalis .

2. There is a longer distance between the frontal and postorbital constriction.

3. There is a narrower postorbital bar, in comparison with the frontal breadth narrowing of this better marked constriction.

4. There is a much larger and rounded infraorbital foramina.

5. Rounded nasal aperture, with the height comparable to the breadth: in M. nivalis the height exceeds the breadth, and the shape is more oval, the index of breadth to height of the nasal aperture in M. erminea is 100.4, while in M. nivalis it is 90.8 .

6. Broader posterior part of $\mathrm{p} 4$ crown: the index of $\mathrm{p} 4$ anterior breadth to $\mathrm{p} 4$ posterior breadth in M. erminea is 82.0 , while in M. nivalis it is 90.0 .

7. Longer $\mathrm{m} 1$ trigonid, with much more marked broadening on the protoconid base: the index of the talonid $\mathrm{m} 1$ length to the trigonid $\mathrm{m} 1$ length is 23.4 in M. erminea, while in M. nivalis it is 41.2 .

8. Stronger reduced talonid of $\mathrm{m} 1$, the index of $\mathrm{m} 1$ talonid breadth to $\mathrm{m} 1$ trigonid breadth in M. erminea is 76.0 , while in M. nivalis it is 92.1 . 


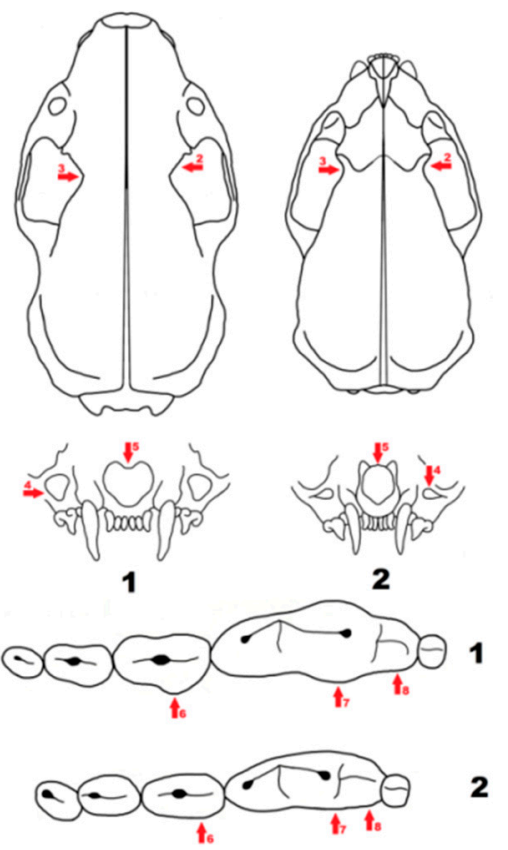

Figure 12. Schematic presentation of the cranial and dental features distinguishing Mustela erminea (1) from Mustela nivalis (2). The features are indicated by red arrows, the numbers next to them correspond to the number of characteristics described in the text.

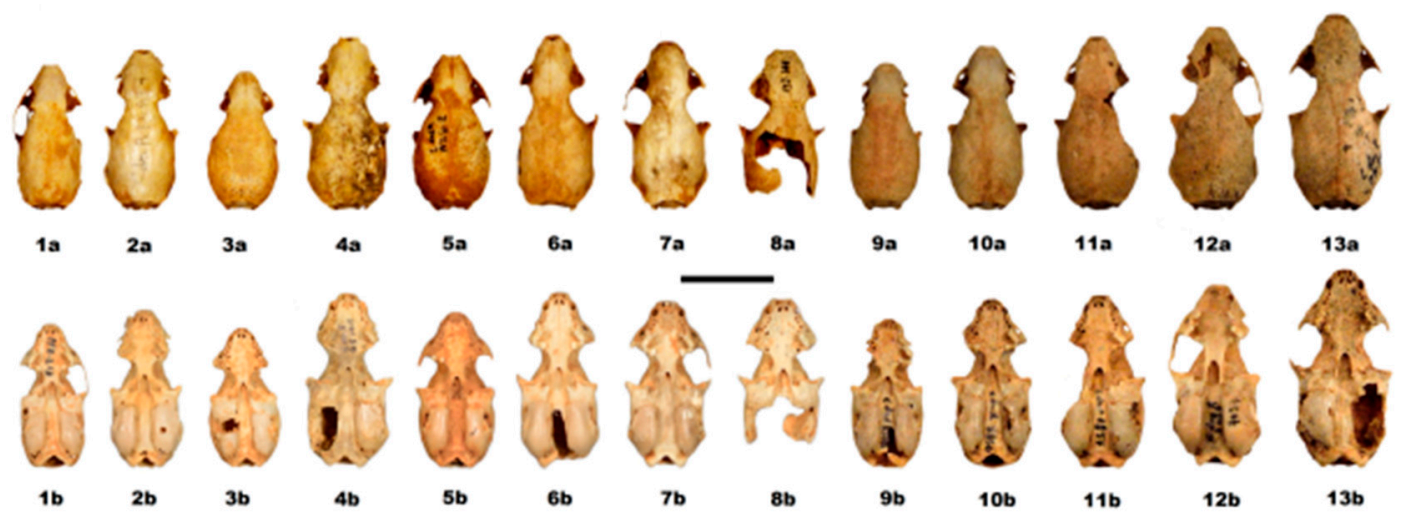

Figure 13. Skulls of Mustela nivalis from Coulet des Roches: M4 217 (1a,b); F5 R 1-1 (2a,b); J5 7 (3); G5 10-1 (4a,b); N3-N4 2 (5a,b); N4 35 (6a,b); M4 548 (7a,b); M3 188 (8a,b); R 8855 (9a,b); R 8856 (10a,b); R 8857 (11a,b); R 7057 (12a,b); and R 2878 (13a,b). Skulls are shown in dorsal (a) and ventral (b) views. Scale bar $20 \mathrm{~mm}$. Photos: C. Triat. 


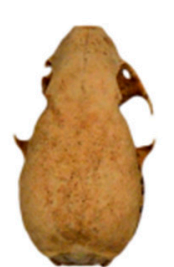

1a

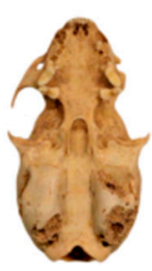

$1 b$

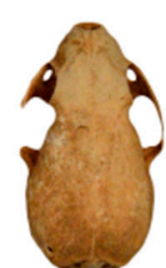

$2 \mathbf{a}$

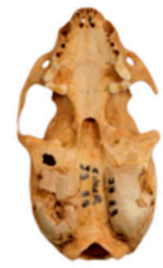

2b

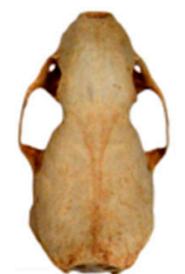

$3 a$

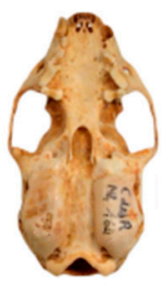

3b

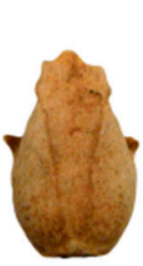

$4 a$

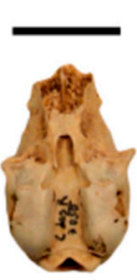

4b

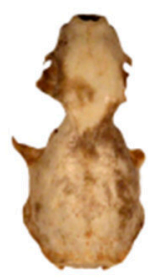

$5 a$

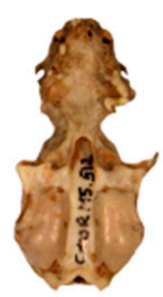

5b

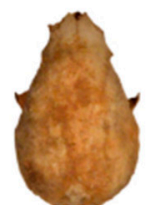

$6 a$

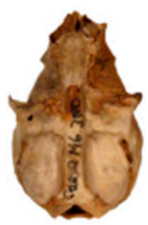

$6 b$

Figure 14. Skulls of Mustela erminea from Coulet des Roches: F5 5 (1a,b); J3 23 (2a,b); N4 164 (3a,b); R 7056 (4a,b); M5 512 (5a,b); and M6 400-4 (6a,b). Skulls are shown in dorsal (a) and ventral (b) view. Scale bar $20 \mathrm{~mm}$. Photos: C. Triat.

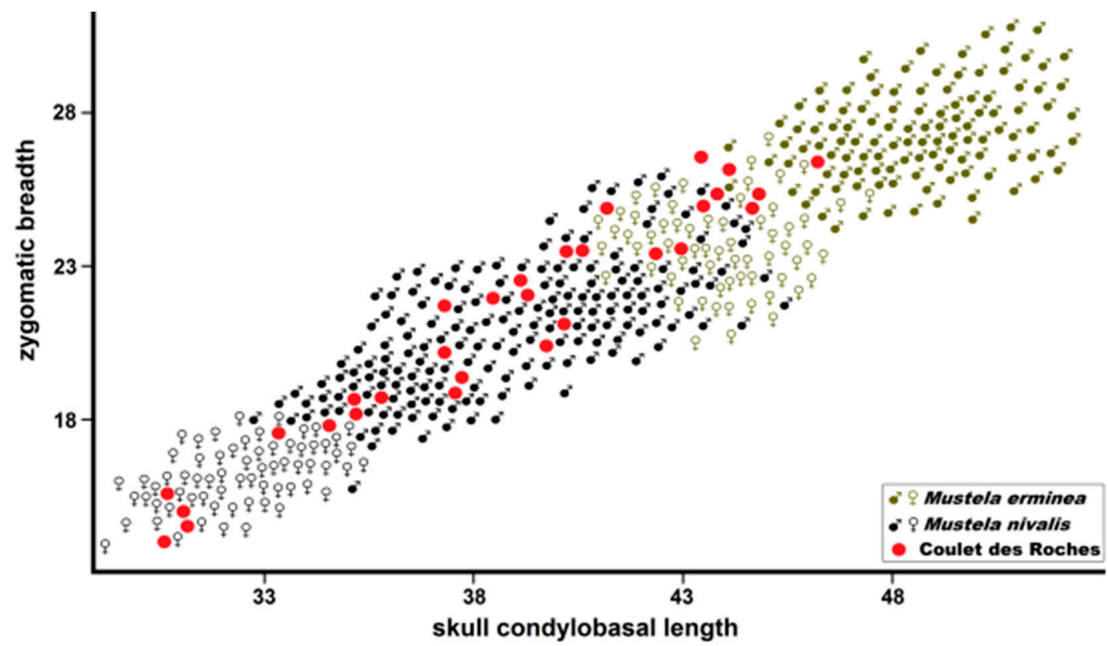

Figure 15. Postorbital skull breadth plotted against condylobasal length in Mustela erminea and Mustela nivalis.

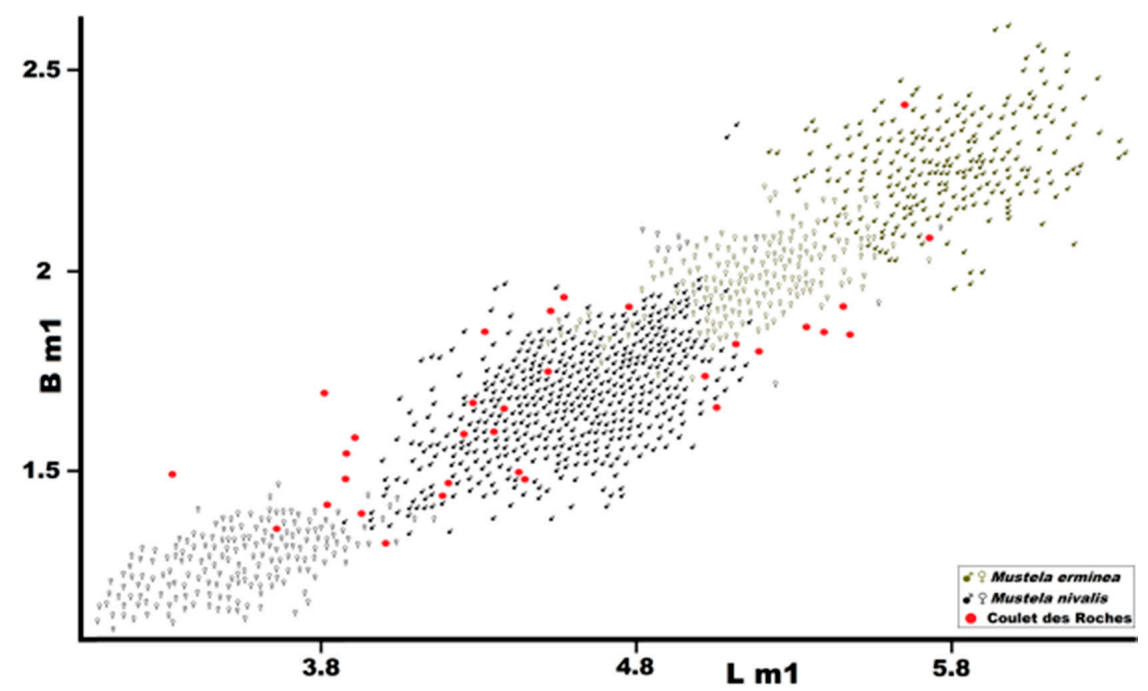

Figure 16. Length of $\mathrm{m} 1$ (L m1) plotted against length of $\mathrm{m} 2$ (L m2) in Mustela erminea and Mustela nivalis. 
Table 9. Skull dimensions of Mustela nivalis from Coulet des Roches (in $\mathrm{mm}$ ). References of measurements in Figure 3. Abbreviations: P, premolar; M, molar; L, length; B, breadth; a, anterior; p, posterior; pr, protocone.

\begin{tabular}{|c|c|c|c|c|c|c|c|c|c|c|c|c|c|c|c|c|c|}
\hline & \multicolumn{10}{|c|}{ LG-GS 2a } & \multicolumn{6}{|c|}{ LGM } & \multirow{2}{*}{$\frac{\text { GI 10-11 }}{\text { N5 }}$} \\
\hline & $\mathbf{R}$ & $\mathbf{R}$ & $\mathbf{R}$ & $\mathbf{R}$ & $\mathbf{R}$ & $\mathbf{R}$ & $\mathbf{R}$ & $\mathbf{R}$ & N4 & N3-N4 & M3 & M4 & M4 & G5 & G4 & F5 & \\
\hline & 2878 & 7344 & 8857 & 8555 & 7057 & 8856 & 8858 & 8870 & 35 & 2 & 188 & 217 & 548 & 10-janv & 11 & R1-1 & $163-1$ \\
\hline & $\sigma^{7}$ & $\sigma^{2}$ & $0^{7}$ & $\sigma^{2}$ & $0^{7}$ & $\sigma^{7}$ & 우 & 우 & $\sigma^{x}$ & q & $\sigma^{x}$ & $0^{7}$ & $\sigma^{x}$ & $0^{x}$ & 우 & $0^{x}$ & $0^{x}$ \\
\hline 1 & 38.9 & - & 34.5 & - & 36.0 & 34.0 & 30.2 & - & 36.2 & 33.2 & - & 31.0 & 34.3 & 36.4 & - & 32.8 & - \\
\hline 2 & 40.3 & - & 35.2 & - & 37.6 & 34.2 & 30.7 & - & 37.3 & 33.4 & - & 31.0 & 35.8 & 37.2 & 30.1 & 33.9 & - \\
\hline 3 & 37.9 & - & 33.0 & - & 34.8 & 31.8 & 28.9 & - & 35.1 & 31.4 & - & 28.9 & 33.1 & 34.8 & 28.2 & 31.9 & - \\
\hline 4 & 15.5 & - & 10.5 & - & 15.7 & 14.5 & 21.6 & - & 20.5 & 15.3 & - & - & 18.3 & 21.6 & - & - & - \\
\hline 5 & 23.6 & - & 24.5 & - & 20.4 & 20.9 & 11.0 & - & 8.5 & 20.3 & - & - & 18.1 & 18.2 & - & - & - \\
\hline 9 & 17.2 & - & 14.6 & 16.8 & 15.5 & 13.5 & 12.5 & - & 16.2 & 14.0 & 14.8 & 12.8 & 15.1 & 16.2 & 11.9 & 13.9 & - \\
\hline 10 & 13.5 & 13.4 & 10.5 & 12.0 & 12.7 & 11.5 & 11.0 & - & 12.6 & 11.6 & 12.0 & 11.0 & 12.3 & 12.3 & 9.7 & 11.2 & - \\
\hline 11 & 7.8 & 7.9 & 7.1 & - & 7.6 & 6.8 & 7.5 & 6.2 & 6.2 & 7.1 & 7.1 & 6.5 & 7.5 & 7.9 & 6.0 & 6.6 & 7.7 \\
\hline 12 & 21.2 & - & - & - & 19.7 & - & - & - & - & 17.4 & - & 15.2 & 18.8 & - & - & - & - \\
\hline 13 & 3.6 & 8.6 & 7.7 & 3.5 & 3.4 & 2.8 & 3.3 & 3.2 & 3.4 & 3.7 & 3.6 & 2.9 & 2.9 & 3.2 & 2.6 & 3.2 & - \\
\hline 14 & 10.1 & 9.1 & 8.4 & 8.2 & 9.3 & 8.1 & 6.6 & 6.9 & 7.6 & 7.6 & 9.0 & 6.9 & 8.4 & 8.7 & 6.0 & 8.7 & - \\
\hline 15 & 12.2 & 13.2 & - & - & 12.3 & 10.6 & - & - & 11.0 & & 12.7 & 10.3 & 10.7 & - & 10.5 & 10.9 & - \\
\hline 16 & 12.7 & 14.0 & 12.2 & - & 12.9 & 11.2 & - & - & 11.6 & 11.7 & 12.6 & 10.4 & 11.8 & 12.3 & 10.8 & 10.9 & - \\
\hline 17 & 4.3 & - & 4.0 & 3.7 & 4.1 & 3.1 & 3.0 & - & 4.0 & 3.6 & 4.2 & 3.5 & 3.8 & 4.1 & 3.1 & 3.8 & - \\
\hline 18 & 10.5 & 10.6 & 9.1 & 9.9 & 10.6 & 8.4 & 7.1 & 8.5 & 8.7 & 9.0 & 9.8 & 8.1 & - & 10.3 & 8.7 & 9.4 & - \\
\hline 20 & 10.7 & - & 9.6 & 9.4 & & 9.5 & 8.6 & - & 9.6 & 8.9 & 9.0 & 8.4 & 9.8 & 9.7 & 8.5 & 9.0 & 10.4 \\
\hline 21 & 8.7 & - & 8.7 & 8.1 & 7.6 & 7.7 & 8.1 & - & 8.2 & 8.4 & 7.6 & 7.0 & 7.5 & 7.7 & 8.0 & 7.1 & 8.0 \\
\hline 22 & 17.9 & - & 16.5 & - & 16.4 & 15.1 & 14.7 & - & 16.3 & 16.3 & - & 13.5 & 15.6 & 16.6 & 15.5 & 14.4 & - \\
\hline 23 & 18.8 & - & 17.0 & - & 18.4 & 15.6 & 14.6 & - & 16.9 & 16.5 & - & 13.8 & 16.7 & 17.8 & 14.0 & 15.6 & - \\
\hline 24 & 10.9 & - & 10.2 & - & 10.0 & 9.4 & 8.3 & - & & 9.3 & - & 9.0 & 9.4 & 9.8 & 8.4 & 8.8 & 9.8 \\
\hline 25 & 3.8 & 3.6 & 3.3 & 3.5 & 3.1 & 3.2 & 2.7 & 2.7 & 3.3 & 2.9 & 3.0 & 2.9 & 3.0 & 3.9 & 3.2 & 3.1 & 3.6 \\
\hline 26 & 3.9 & 3.4 & 3.6 & 3.7 & 3.4 & 3.5 & 2.8 & 3.4 & 3.6 & 3.1 & 3.6 & 2.6 & 3.6 & 3.5 & 2.9 & 2.6 & 3.7 \\
\hline 27 & 6.2 & - & 6.1 & - & 5.7 & 4.7 & 4.6 & - & & 5.4 & - & 5.0 & 5.1 & 5.1 & 4.6 & 5.0 & 5.8 \\
\hline 28 & 4.9 & - & 4.7 & - & 4.5 & 5.0 & 4.5 & - & & 4.8 & - & 4.0 & 4.9 & 4.1 & 4.0 & 4.2 & 4.8 \\
\hline 29 & 13.3 & - & 11.8 & - & 13.0 & 11.5 & 10.5 & - & 12.3 & 11.5 & - & 10.6 & 12.1 & 10.7 & 9.9 & 11.4 & - \\
\hline 30 & 7.8 & - & 6.8 & - & 6.9 & 6.3 & 6.1 & - & 7.2 & 6.7 & - & 5.4 & 6.4 & 7.7 & 5.1 & 6.4 & - \\
\hline P3 L & - & 2.5 & - & - & 1.9 & - & 2.2 & 2.1 & - & - & - & 1.8 & - & 2.5 & - & 1.9 & - \\
\hline P3 B & - & 1.1 & - & - & 1.0 & - & 1.0 & 1.0 & - & - & - & 1.1 & - & 1.3 & - & 0.9 & - \\
\hline P4 L & 4.3 & 4.5 & 3.8 & - & 3.9 & 3.5 & 3.9 & 3.4 & 4.1 & - & 4.0 & 3.7 & 4.0 & 4.1 & - & 4.0 & 4.5 \\
\hline P4 L pr & 1.1 & 1.2 & 0.8 & - & 0.7 & 0.7 & 0.7 & 1.0 & 1.1 & - & 0.9 & 0.7 & 0.9 & 2.4 & - & 1.6 & 0.9 \\
\hline $\mathrm{P} 4 \mathrm{Ba}$ & 2.4 & 2.4 & 2.2 & - & 2.2 & 2.0 & 2.3 & 2.2 & 2.2 & - & 2.2 & 2.1 & 2.3 & 2.5 & - & 2.4 & 2.4 \\
\hline P4 Bp & 1.5 & 1.5 & 1.3 & - & 1.5 & 1.3 & 1.0 & 1.2 & 1.3 & - & 1.5 & 1.3 & 1.4 & 1.2 & - & 1.2 & 1.5 \\
\hline M1 L & 3.2 & 3.5 & 3.0 & - & 3.0 & 3.1 & 3.0 & - & 3.2 & 2.8 & - & 2.7 & 3.3 & - & - & 3.2 & - \\
\hline M1 B1 & 1.3 & 1.2 & 1.1 & - & 1.5 & 1.2 & 1.4 & - & 1.2 & 1.4 & - & 1.5 & 1.5 & - & - & 1.1 & - \\
\hline M1 B2 & 1.5 & 1.5 & 1.2 & - & 1.4 & 1.1 & 1.0 & - & 1.7 & 1.3 & - & 1.2 & 1.2 & - & - & 1.1 & - \\
\hline M1 B3 & 1.8 & 1.8 & 1.4 & - & 1.4 & 1.4 & 1.1 & - & 1.4 & 1.5 & - & 1.4 & 1.8 & - & - & 1.5 & - \\
\hline
\end{tabular}

Table 10. Mandible dimensions of Mustela nivalis from Coulet des Roches (in mm). References of measurements in Figures 4 and 5. Abbreviations: L, left; R, right; p, premolar; m, molar; L, length; B, breadth; a, anterior; p, posterior; tri, trigonid; tal, talonid.

\begin{tabular}{|c|c|c|c|c|c|c|c|c|c|c|c|c|c|c|c|c|c|c|}
\hline & \multicolumn{18}{|c|}{ LG-GS 2a } \\
\hline & $\mathbf{R}$ & $\mathbf{R}$ & $\mathbf{R}$ & $\mathbf{R}$ & $\mathbf{R}$ & $\mathbf{R}$ & $\mathbf{R}$ & $\mathbf{R}$ & $\mathbf{R}$ & $\mathbf{R}$ & $\mathbf{R}$ & $\mathbf{R}$ & $\mathbf{R}$ & $\mathbf{R}$ & M3 & M5 & M3-4 & M3-4 \\
\hline & 3034 & 3035 & 3036 & 3038 & 3039 & 3719 & 3059 & 8858 & 8858 & 3060 & 9294 & 9295 & 9306 & 9326 & $61-4$ & $41-4$ & & \\
\hline & $0^{2}$ & $\sigma^{2}$ & $0^{7}$ & q & ㅇ & $\sigma^{7}$ & $0^{7}$ & ㅇ & q & $\sigma^{7}$ & $0^{7}$ & 우 & q & $\sigma^{x}$ & $0^{7}$ & q & $\sigma^{7}$ & $\sigma^{2}$ \\
\hline 1 & 17.0 & 14.3 & 16.3 & 16.2 & - & 20.8 & 18.2 & 14.9 & 15.0 & 19.5 & 20.3 & 18.7 & 16.8 & & 19.4 & 17.2 & 15.7 & - \\
\hline 2 & 16.1 & 13.3 & 15.5 & 15.4 & - & 19.9 & 17.0 & 14.3 & 14.3 & 18.4 & 19.3 & 17.2 & 15.8 & 17.4 & 18.4 & 15.8 & 14.7 & - \\
\hline 3 & 11.4 & 9.7 & 10.6 & 10.9 & - & 13.6 & 11.9 & 10.1 & 10.2 & 13.1 & 13.1 & 11.5 & 10.8 & 13.2 & 12.3 & 12.1 & 10.8 & - \\
\hline 4 & 10.3 & - & 9.6 & 11.0 & - & 13.3 & 11.7 & 10.2 & 9.9 & 12.4 & 12.2 & 11.6 & 10.4 & 11.7 & 11.5 & 11.3 & 8.8 & - \\
\hline 5 & 8.9 & - & - & 8.9 & 8.3 & 10.2 & 9.9 & 8.3 & 8.1 & 10.0 & 10.0 & 9.4 & 9.0 & 8.9 & 9.4 & 8.9 & 8.5 & 9.5 \\
\hline 6 & 3.7 & - & 4.4 & 4.4 & 4.0 & 5.3 & 4.2 & 3,4 & 3.5 & 4.6 & 4.8 & 4.3 & 3.4 & 3.8 & 9.7 & 4.2 & 3.9 & 4.8 \\
\hline 7 & 4.5 & 4.9 & - & 4.8 & 4.1 & 5.3 & 5.5 & 4.7 & 5.3 & 4.9 & 5.3 & 4.7 & 4.7 & 5.4 & 5.1 & 4.3 & 4.4 & 5.1 \\
\hline 8 & 1.9 & - & 1.6 & 1.5 & 1.4 & 2.1 & 1.5 & 1.8 & 1.9 & 2.5 & 1.9 & 1.2 & 1.5 & - & 1.6 & 1.7 & 1.2 & - \\
\hline 10 & 8.1 & 8.8 & - & 8.2 & - & - & - & 6.8 & 6.9 & 9.2 & 10.3 & 9.1 & 8.6 & - & 9.1 & 8.3 & 7.6 & - \\
\hline 12 & 3.4 & - & 3.2 & 3.0 & 2.8 & 4.3 & - & 2.5 & 2.7 & 3.5 & 4.3 & 4.0 & 2.9 & 3.6 & 4.6 & 3.6 & 2.9 & - \\
\hline 14 & 3.2 & - & 3.1 & 3.1 & 3.1 & 4.2 & 3.4 & 2.9 & 3.3 & 4.0 & 4.2 & 3.7 & 3.3 & 4.4 & 3.4 & & 2.8 & 3.4 \\
\hline 17 & & 4.7 & 3.4 & 3.5 & 3.3 & - & 4.3 & 3.3 & 3.2 & 4.7 & 4.5 & 4.8 & 4.1 & - & 4.5 & 4.2 & 3.7 & 3.8 \\
\hline p2 L & & - & - & - & 1.0 & - & - & 0.8 & - & 1.2 & - & 1.2 & - & - & - & - & 1.5 & - \\
\hline
\end{tabular}


Table 10. Cont.

\begin{tabular}{|c|c|c|c|c|c|c|c|c|c|c|c|c|c|c|c|c|c|c|}
\hline p2 B & & - & - & - & 0.9 & - & - & 0.5 & - & 0.8 & - & 0.8 & - & - & - & - & 0.7 & - \\
\hline p3 L & & - & 2.3 & - & - & 1.9 & - & 1.3 & - & - & - & 2.1 & 1.4 & - & - & - & - & 1.6 \\
\hline p3 B & & - & 0.9 & - & - & 1.1 & - & 0.9 & - & - & - & 1.1 & 0.9 & - & - & - & - & 1.0 \\
\hline $\mathrm{p} 4 \mathrm{~L}$ & 2.2 & - & 2.1 & - & 1.6 & 2.6 & - & 1.9 & 1.9 & 2.4 & - & 2.3 & 2.3 & - & - & 2.0 & 2.0 & 2.3 \\
\hline $\mathrm{p} 4 \mathrm{Ba}$ & 0.7 & - & 0.8 & - & 0.0 & 1.1 & - & 0.9 & 0.7 & 0.9 & - & 1.2 & 1.1 & - & - & 0.9 & 0.9 & 1.0 \\
\hline $\mathrm{p} 4 \mathrm{Bp}$ & 1.1 & - & 0.9 & - & 1.2 & 1.5 & - & 1.1 & 0.8 & 1.2 & - & 1.4 & 1.2 & - & - & 1.1 & 1.0 & 1.4 \\
\hline $\mathrm{m} 1 \mathrm{~L}$ & 4.0 & 4.3 & - & 3.9 & 3.3 & 4.4 & 4.5 & 3.7 & 3.6 & 4.4 & 4.3 & 3.8 & 3.9 & 4.6 & 4.2 & 3.9 & 4.5 & 4.2 \\
\hline $\mathrm{m} 1 \mathrm{~L}$ tri & 2.7 & 2.9 & - & 2.8 & 2.5 & 3.3 & 3.3 & 2.7 & 2.7 & 3.4 & 3.2 & 3.3 & 2.8 & 3.3 & 2.9 & 2.7 & 2.9 & 3.0 \\
\hline $\mathrm{m} 1 \mathrm{~L}$ tal & 1.3 & 1.3 & - & 0.9 & 0.7 & 1.1 & 1.2 & 0.9 & 1.0 & 1.3 & 1.3 & 0.8 & 1.1 & 1.3 & 1.5 & 1.4 & 0.7 & 0.9 \\
\hline m1 B tri & 1.3 & 1.6 & - & 1.6 & 1.5 & 1.7 & 1.9 & 1.4 & 1.3 & 1.5 & 1.6 & 1.7 & 1.5 & 1.9 & 1.4 & 1.5 & 1.5 & 1.5 \\
\hline m1 B tal & 1.2 & 1.5 & - & 1.5 & 1.3 & 1.5 & 1.5 & 1.1 & 1.1 & 1.3 & 1.3 & 1.3 & 1.3 & 1.5 & 1.3 & 1.2 & 1.2 & 1.4 \\
\hline $\mathrm{m} 2 \mathrm{~L}$ & 0.8 & - & - & - & - & 1.1 & - & - & - & 0.6 & - & 0.7 & - & - & - & 0.5 & 0.7 & - \\
\hline \multirow[t]{5}{*}{$\mathrm{m} 2 \mathrm{~B}$} & 0.9 & - & - & - & - & 1.2 & - & - & - & 0.7 & - & 0.7 & - & - & - & 0.6 & 1.1 & - \\
\hline & \multicolumn{7}{|c|}{ LGM } & \multicolumn{3}{|c|}{ GI 3} & \multicolumn{3}{|c|}{ GI 3? } & & & & & \\
\hline & N5-6 & M4 & M4 & M4 & G5 & F5 & F5 & L6 & L6 & L5 & M6-7 & M6- & M7 & & & & & \\
\hline & R1 & 217 & 458 & 458 & & R1-2 & R5-8 & R10-1 & R10-1 & 53-1 & R62-1 & R57- & & & & & & \\
\hline & $0^{\pi}$ & $\sigma^{2}$ & $0^{x}$ & $\sigma^{x}$ & & $0^{x}$ & $0^{x}$ & $0^{x}$ & $0^{x}$ & $\sigma^{7}$ & $0^{x}$ & $\sigma^{x}$ & & & & & & \\
\hline 1 & 17.8 & 15.7 & 18.5 & - & 16.6 & 17.3 & 17.2 & 19.4 & - & 18.8 & 20.7 & 20.8 & & & & & & \\
\hline 2 & 16.6 & 15.0 & 17.0 & - & 15.9 & 16.1 & 16.1 & 18.1 & - & 17.8 & 19.3 & 19.3 & & & & & & \\
\hline 3 & 11.5 & 10.8 & 12.5 & - & 11.3 & 11.4 & 11.0 & 12.0 & - & 13.1 & 13.3 & 13.7 & & & & & & \\
\hline 4 & 10.7 & 10.1 & 11.4 & - & 10.6 & 10.9 & 11.0 & 12.1 & - & 11.7 & 12.6 & 12.9 & & & & & & \\
\hline 5 & 9.5 & 8.6 & 9.3 & - & 8.5 & 8.8 & 9.5 & 9.9 & - & - & 11.1 & 10.2 & & & & & & \\
\hline 6 & 8.8 & 4.3 & 4.3 & - & 3.9 & 4.7 & 4.0 & 4.8 & - & - & 5.1 & 4.4 & & & & & & \\
\hline 7 & 5.0 & 4.6 & 5.0 & - & 4.5 & 4.5 & 4.6 & 5.8 & - & 6.4 & 6.0 & 6.5 & & & & & & \\
\hline 8 & 1.9 & 1.1 & 1.4 & - & 1.6 & 2.4 & 2.1 & 2.2 & - & & 1.9 & 0.9 & & & & & & \\
\hline 10 & 8.5 & 7.6 & 8.8 & - & 8.5 & 8.0 & 8.0 & 10.1 & - & 10.8 & 10.2 & 10.3 & & & & & & \\
\hline 12 & 3.9 & 3.1 & 3.6 & - & 4.0 & 3.3 & 3.9 & 3.7 & - & & 3.3 & 4.5 & & & & & & \\
\hline 14 & 3.4 & 2.9 & 3.9 & - & 3.5 & 3.2 & 3.4 & 3.8 & - & 3.6 & 3.9 & 3.8 & & & & & & \\
\hline 17 & 4.4 & 4.0 & 4.5 & - & 4.0 & 4.1 & 4.0 & 4.5 & - & 5.9 & 4.9 & 5.0 & & & & & & \\
\hline p2 L & - & 0.9 & 0.9 & 1.0 & - & 0.7 & - & - & - & - & - & - & & & & & & \\
\hline p2 B & - & 0.7 & 0.7 & 1.0 & - & 0.7 & - & - & - & - & - & - & & & & & & \\
\hline p3 L & - & - & 1.5 & 1.7 & 1.6 & 1.9 & 2.3 & - & - & - & - & - & & & & & & \\
\hline p3 B & - & - & 1.0 & 1.0 & 1.2 & 1.0 & 1.1 & - & - & - & - & - & & & & & & \\
\hline p4 L & 2.1 & 2.0 & 2.0 & 2.0 & - & 2.0 & 2.3 & 2.2 & - & - & - & 2.6 & & & & & & \\
\hline $\mathrm{p} 4 \mathrm{Ba}$ & 0.8 & 1.0 & 1.1 & 1.1 & - & 0.8 & 1.1 & 1.1 & - & - & - & 1.2 & & & & & & \\
\hline $\mathrm{p} 4 \mathrm{Bp}$ & 1.3 & 1.3 & 1.2 & 1.3 & - & 0.9 & 1.1 & 1.1 & - & - & - & 1.4 & & & & & & \\
\hline $\mathrm{m} 1 \mathrm{~L}$ & 4.3 & 4.1 & 4.2 & 4.3 & 4.0 & 4.0 & 4.4 & 4.8 & 5.0 & 5.4 & 5.1 & 5.3 & & & & & & \\
\hline $\mathrm{m} 1 \mathrm{~L}$ tri & 3.1 & 2.7 & 3.0 & 3.3 & 2.6 & 2.9 & 3.3 & 3.5 & 3.3 & 4.0 & 3.6 & 3.6 & & & & & & \\
\hline $\mathrm{m} 1 \mathrm{~L}$ tal & 1.1 & 0.8 & 1.1 & 1.1 & 1.2 & 1.5 & 1.5 & 1.6 & 1.5 & 1.8 & 1.4 & 1.3 & & & & & & \\
\hline m1 B tri & 1.9 & 1.4 & 1.7 & 1.4 & 1.4 & 1.4 & 1.4 & 1.7 & 1.8 & 1.9 & 1.8 & 2.0 & & & & & & \\
\hline m1 B tal & 1.7 & 1.2 & 1.5 & 1.3 & 1.3 & 1.2 & 1.2 & 1.5 & 1.6 & 1.8 & 1.6 & 1.7 & & & & & & \\
\hline m2 L & 1.0 & - & 1.4 & - & 0.9 & 1.0 & - & 1.0 & 1.1 & 1.0 & - & - & & & & & & \\
\hline $\mathrm{m} 2 \mathrm{~B}$ & 0.8 & - & 0.9 & - & 0.7 & 1.0 & - & 0.7 & 1.1 & 0.9 & - & - & & & & & & \\
\hline
\end{tabular}

\subsubsection{Taxonomy}

The examined mustelid material from Coulet des Roches represents three species: M. putorius, M. erminea and M. nivalis. M. putorius is represented by a total number of 66 bones, belonging to four males (NISP $=66, \mathrm{MNI}=4$ ). The $M$. erminea material is represented by 99 bones, belonging to eight males and six females (NISP $=99, \mathrm{MNI}=14,80^{7} 0^{7}, 6$ \%). In total, 272 bones were assigned to the most abundant species, M. nivalis, represented by at least 48 individuals: 18 from excavated sediments, 5 from the Late Glacial-GS 2a, 21 from the Pleniglacial-LGM, 3 from GI 3 and 1 from GI 10-11 (MIS 3). There are 32 males, 12 females, and 4 indeterminate specimens (NISP $=272, \mathrm{MNI}=40,38 \mathrm{o}^{7} \mathrm{o}^{7}, 12$ 우, and 4 indeterminate) [17].

These species are widespread throughout the European Pleistocene and have been recorded in numerous paleontological sites [21,59]. In addition, archaeological excavations have yielded many single occurrences of mustelid species of different ages [21,59]. However, despite their relative abundance in faunal assemblages, these carnivores are usually represented by a limited number of individuals and remains, for several reasons. First, during earlier excavations (especially those carried out by archaeologists), sediments were either not sieved or washed at all, or only to a very limited extent [60]. Another reason is the small size of mustelids and the high probability of mistaking their remains, especially postcranial elements, for rodents, bats or even insectivores. They are often simply overlooked during taxonomic analysis, or mislabeled. The third reason is that they are too 
small to be a valued prey for humans and were usually only introduced to archaeological sites accidentally $[21,59,60]$. Birds of prey, owls and larger carnivores, e.g., Vulpes vulpes, or even larger mustelids, rather than humans, are responsible for the accumulation of mustelid remains. Despite their agility and ferocious defensive behavior, these small carnivores are relatively vulnerable to attacks by their larger counterparts $[61,62]$.

Forest polecat specimens from Late Pleistocene sites in Europe (including France) have been included in M. putorius putorius [21,39,40]. Their size and morphology are within the variability ranges of recent nominative subspecies occurring in Europe [39]. Nowadays, this species is characterized by smaller dimensional variability than the steppe polecat, and individuals from different regions of Europe reach similar sizes. Only Scandinavian populations are characterized by slightly larger dimensions [32,39]. The forest polecat prey spectrum is more diversified than the steppe polecat diet. The diet consists mainly of small mammals and amphibians (in the United Kingdom, mostly rabbits) with high seasonal variation $[39,63,64]$. This species is currently associated with the fourth and sixth plant zones [65]. It shows preference for aquatic and marshy areas, and usually inhabits the edges of large forest complexes and mosaic areas. On the other hand, unlike the steppe polecat, it avoids large open steppe-like areas. It also shows strong synanthropization [63].

The steppe polecat is closely associated with open areas and steppes (with the sixth and seventh plant zones) and, unlike the forest polecat, it does not undergo synanthropization $[39,40,65]$. The remains of $M$. eversmanii in cave sediments generally occur with a large number of rodents from the genera Spermophilus Cuvier, 1825, Cricetus Leske, 1799, Cricetullus Milne-Edwards, 1867 and Microtus Schrank, 1798. This confirms the existence of open steppe areas during the deposition period [33,40,65].

Polecats are quite rare species in cave deposits and are usually represented by a small number of individuals. This is mainly due to the type of environment they inhabit. Both species avoid rocky habitats $[32,39,40]$. Polecats are characterized by a weaker climbing ability and, unlike martens, they rarely enter caves in search of food. On account of their relatively large size and aggressive defense (among others, the ability to expel foul-smelling substances from perianal areas), they rarely fall prey to other predators. Water transport and the role of caves as traps also played an accumulating role. Humans also played a role, using some animals for fur [32,59]. The species is relatively rarely reported in the Pleistocene deposits of France. It is absent from the faunal lists of the rare sites with Pleniglacial and Tardiglacial assemblages in the southeast of France, with the exception of Aven des Planes, where it is represented by a female skull with mandibles from layer E of GS 2a [66]. In this context, the presence of four individuals in Coulet des Roches is noteworthy.

Complete skulls of the smallest mustelids, the stoat and common weasel, are rare finds, especially for the Late Pleistocene. Abundant cranial material from the Late Glacial southeastern French locality Coulet des Roches is unique for two major reasons. Firstly, it is conducive to a detailed metric and morphological analysis, and, secondly, in one locality, three different "eco-types" of M. nivalis are represented.

The stoat, M. erminea, has been found in two studied French localities. Five individuals come from LGM levels in Coulet des Roches and the species was also found in LG levels dated to GS 2a in Aven de Planeyssard (Vaucluse). Remains of the stoat are much less frequent than those of the common weasel. This is a typical situation for mustelids, where larger species are outnumbered by their smaller cousins. Females are more frequent than males (6:4); and the skulls belong to quite young individuals, mostly from age classes I and II.

The weasel, M. nivalis, has been found in LG and LGM layers from Coulet des Roches and in LG layers from Aven des Planes, as well as two Holocene Vauclusian localities: Mont Ventoux 4 and Dolmen Goult (Bronze Age) [66]. In Coulet des Roches, the fossil material from weasels heavily outnumbered stoats, and males are much more frequent than females. Most remains belong to young and adult specimens with unworn teeth and the postcranial material is also well preserved.

Due to its circumboreal Holarctic distribution, $M$. nivalis shows high size variation, which generally does not follow Bergmann's rule. The variability of this subspecies has recently 
been summarized by Abramov and Baryshnikov [67], who divided weasels into three main groups (Figure 17):

(a) The largest, long-tailed group boccamela-numidica, the so-called "Mediterranean or Transcaucasian weasels", which occur along the Mediterranean coast, are regarded as the most primitive weasels, the direct descendants of Middle Pleistocene ancestors;

(b) Average-sized, shorter-tailed weasels form the nivalis-vulgaris group, the so-called "common weasels", which recently inhabited most of Europe; and

(c) Dwarf and small, short-tailed weasels form the pygmaea-rixosa group, the so-called "least or pygmy weasels", which occupy the Northern hemisphere and cold and snowy climates $[31,66]$.

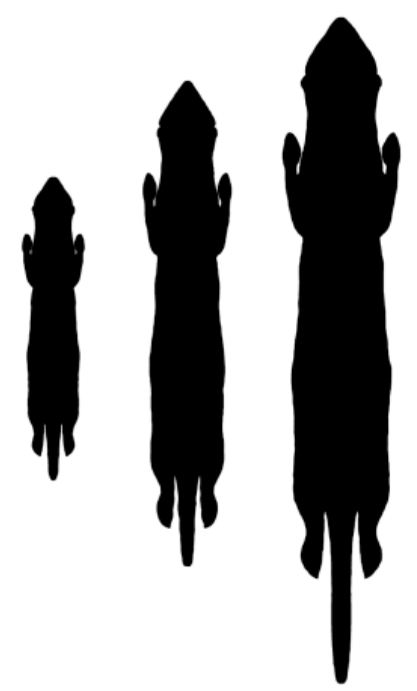

Figure 17. Three basic types of Mustela nivalis weasel, examined as "eco-types": (left) dwarf, short-tailed pygmy (least) weasel (eg., M4 217) forming the pygmaea-rixosa group, characteristic of northern, cold climates; (middle) average, short-tailed common weasel (eg., R 8857, R 7057) from the vulgaris-nivalis group, typical of temperate climates; and (right) large, long-tailed Transcaucasian weasel from the boccamela-numidica group, characteristic of warm climates. Note size differences. After King and Powell [62] and Abramov and Baryshnikov [67], modified.

Large weasels from the boccamela-numidica group had a much wider geographical distribution during the Middle Pleistocene, but, due to pressure from ice sheets, they were pushed southwards, to Southeast Asia and North Africa. However, some isolated populations (e.g., M. nivalis subpalmata in Egypt) remained and evolved in isolation from the rest of the weasels [68]. During glaciations, the size of Mustela nivalis decreased rapidly and smaller, better adapted animals to cold and snowy climatic conditions came from the east. The smallest pygmy weasels (form known in the literature as "minuta") co-existed alongside this species. The occurrence of the pygmy weasel in Western Europe is typical during glacial maximums, when this form even inhabited the Iberian Peninsula. In the postglacial period, when the climate became warmer, this highly evolved form was replaced by average-sized, more opportunistic weasels from the nivalis-vulgaris group. In Western Europe, dwarf weasels only survived in small isolated refuges, for example, high-altitude habitats in the Alps and Pyrenees [62,67].

Recently, two weasel subspecies occurred in France: a smaller, common weasel with a relatively short tail, M. nivalis vulgaris Erxleben, 1777, which inhabited most of France and the second, larger, long-tailed Transcaucasian weasel, M. nivalis boccamela Bechstein, 1800, which occurred in Southern France [32].

The M. nivalis specimens from the Late Glacial locality Coulet des Roches are characterized by a generally small, or at most medium size (condylobasal length (CBL) $0^{7} 0^{7}=36.0 \mathrm{~mm}$, $\mathrm{CBL}+9 \%=31.7 \mathrm{~mm}$ ). They are slightly smaller than the vulgaris subspecies that occurred recently 
in most of France (CBL $0^{7} 0^{7}=38.8 \mathrm{~mm}$ [31.5-43.0] mm, CBL of $=33.2 \mathrm{~mm}$ [25.8-36.0] mm) [31] (Figure 15). This suggests that they might represent the population pushed further south (or further east) during the LGM, and that they re-colonized this area after the disappearance of the ice sheet. The slightly smaller size of the individuals from Coulet des Roches points to still unstable climatic conditions and some colder periods during the accumulation of sediments. The medium size of the mandibles and long bones is generally comparable to the dimensions of weasels from other Late Pleistocene French localities, e.g. Coudes, La Colombière, La Baume de Gigny, Lourdes Calvaire or Grotte du bois de Cantet [35,36,52,69] (Table 11).

Table 11. Lower carnassial (m1) size comparison between the weasel Mustela nivalis from different late Middle Pleistocene, Late Pleistocene and Holocene localities. Abbreviations: H, Holocene; Min, minimum; Max, maximum; $\mathrm{m}$, average; SD, standard deviation; ${ }^{1}$ this work; ${ }^{2}[36]{ }^{3}{ }^{[35]}{ }^{4}{ }^{4}[69] ;{ }^{5}[34]$; and ${ }^{6}[67]$.

\begin{tabular}{|c|c|c|c|c|c|c|}
\hline Site & Sex & $n$ & Min & Max & m & SD \\
\hline Coulet des Roches ${ }^{1}$ & $0^{7}$ & 13 & 4.01 & 4.53 & 4.28 & 0.14 \\
\hline Dolmen Goult $(\mathrm{H})^{1}$ & $\sigma^{7}$ & 2 & 4.77 & 4.79 & 4.78 & - \\
\hline Les Planes $(\mathrm{H})^{1}$ & $\sigma^{7}$ & 1 & - & - & 4.51 & - \\
\hline Grotte de Gerde ${ }^{2}$ & $\sigma^{7}$ & 18 & 4.00 & 4.70 & 4.32 & 0.24 \\
\hline Coudes ${ }^{3}$ & $\sigma^{7}$ & 2 & 4.23 & 4.49 & 4.36 & - \\
\hline Lourdes Calvaire $^{2}$ & $0^{7}$ & 3 & 4.00 & 4.00 & 4.00 & - \\
\hline Grotte du bois de Cantet 4 & $\sigma^{7}$ & 2 & 4.30 & 4.50 & 4.35 & - \\
\hline La Colombière ${ }^{3}$ & $\sigma^{7}$ & 23 & 3.84 & 4.49 & 4.14 & 0.20 \\
\hline La Baume de Gigny 5 & $0^{x}$ & 24 & 3.78 & 4.42 & 4.10 & 0.18 \\
\hline Abîmes de la Fage ${ }^{3}$ & $\sigma^{7}$ & 114 & 4.01 & 4.96 & 4.40 & 0.20 \\
\hline France recent (vulgaris) ${ }^{6}$ & $0^{x}$ & 22 & - & - & 4.21 & 0.20 \\
\hline South Europe (boccamela) ${ }^{6}$ & $0^{x}$ & 17 & - & - & 4.60 & 0.10 \\
\hline Coulet des Roches ${ }^{1}$ & q & 7 & 3.33 & 3.91 & 3.70 & 0.21 \\
\hline Coulet des Roches $(\mathrm{H})^{1}$ & 우 & 2 & 3.82 & 3.93 & 3.88 & - \\
\hline Grotte de Gerde 2 & 우 & 8 & 3.60 & 3.90 & 3.75 & 0.09 \\
\hline Coudes ${ }^{3}$ & 우 & 2 & 3.46 & 3.84 & 3.65 & - \\
\hline Lourdes Calvaire $^{2}$ & 우 & 1 & - & - & 3.50 & - \\
\hline La Colombière ${ }^{3}$ & 우 & 30 & 3.26 & 3.83 & 3.57 & 0.16 \\
\hline La Baume de Gigny 5 & 우 & 25 & 3.00 & 3.78 & 3.48 & 0.20 \\
\hline Abîmes de la Fage ${ }^{3}$ & 우 & 69 & 3.24 & 3.98 & 3.69 & 0.21 \\
\hline
\end{tabular}

The morphology of this male suggests a fully adult age, which is confirmed by fully fused sutures, good development of postorbital constriction and slightly worn teeth and is also comparable to the morphology of pygmy weasel skulls. In general, the skull of the smallest weasels still retains some juvenile features in adults. As Heptner and Naumov ([32] p. 969) stated: "On the whole, the weasel skull, especially that of the smallest forms (rixosa group) has infantile features in comparison to other closely related species of the genus (ermine, Siberian weasel). These are expressed in the relatively large size of the entire cranial portion, in the size of its anterior part (on the extension of the zygomatic arches-the frontal part), in the unusual shortening of the facial part, the weak development of the crests and in general sculpturing".

Only two specimens are larger than the others. The first is male no. $\mathrm{R} 2878(\mathrm{CBL}=40.3 \mathrm{~mm})$, which is larger than the other individuals, but its dimensions also correspond well to the size range of the vulgaris subspecies (CBL $0^{n} \sigma^{x}=38.8 \mathrm{~mm}[31.5-43.0] \mathrm{mm}$ ), rather than to the size range of the boccamela type (CBL $\circ^{7} \sigma^{7}=42.1 \mathrm{~mm}[41.0-46.2] \mathrm{mm}$ ). This suggests that almost all the weasel material from Coulet des Roches (apart from the pygmy male M4 217) belongs to average-sized weasels from the vulgaris group, which inhabited this area until recently. Only the large, massive mandibles from the Holocene localities Dolmen Goult (JZ 431) and Les Planes can be considered as possible boccamela specimens, but due to the limited number of specimens, this is rather risky. 
The second individual is represented by a complete left mandible (collection No. M3-M4 1-1) of a large male. It presents a unique morphology with features typical of both the stoat and the weasel. In this specific case, size criteria are unusable, as the dimensions are in the size range of large male weasels and small female stoats. Usually, in such cases, if the length of the lower carnassial is more than $5 \mathrm{~mm}$, the specimen is attributed to the stoat rather to the weasel. However, in the above case, this is not clear. The lower carnassial is characterized by a quite short and clearly reduced talonid, which is more typical of the stoat. The second aspect is the relatively low and slender body of the mandible, which is usually more typical of females than males.

However, multivariate analysis showed that this is rather a large boccamela type male. This large weasel is characterized by the occurrence of such unusual male individuals, with a morphology typical of both forms. In addition, the relatively narrow $\mathrm{m} 1$ is weasel-like rather than stoat-like and its size is much more typical of the boccamela type $(\mathrm{Lm} 1=4.60 \mathrm{~mm})$ than the vulgaris type $(\mathrm{Lm} 1=4.21 \mathrm{~mm})$ [67].

This factor contributes to the uniqueness of the Coulet des Roches site. It seems that this site was located on the border of a contact zone of two different weasel types: vulgaris and boccamela. Recently, the boccamela group weasel only inhabits the southernmost part of France, around the Mediterranean coast, while the vulgaris type is widely distributed across France. In a few isolated populations, at high altitudes in the Alps and Pyrenees, relict rixosa type populations also occur; the so-called "Ice Age survivor". The contact zone between the vulgaris and boccamela types is not clearly defined, and weasels from both populations cross each other's boundaries and sometimes occur in the same area. A similar situation could have occurred at Coulet des Roches.

Similar to the weasel, the stoat also shows high geographical differentiation, but the level of this variability is less pronounced than for the weasel [62]. It seems that Late Pleistocene stoats were smaller than Holocene and recent stoats, but the limited number of specimens rules out detailed analysis. The stoat is always much less frequent than the weasel in the same localities, and Roches des Planes is no exception. M. erminea fossil material from this locality, as well as the female skull from Aven de Planeyssard, are smaller than the modern, large stoat M. erminea erminea Linnaeus, 1758, which still inhabits France (CBL $0^{7} \sigma^{7}$ from Coulet des Roches $=45.3 \mathrm{~mm}$ [44.2-46.3] mm vs. recent M. erminea $o^{7} o^{7}$ Average $=49.6 \mathrm{~mm}$ ) [70].

The stoat material from Coulet des Roches is more comparable to the medium-sized subspecies M. erminea aestiva Kerr, 1792, which now occurs mostly in Central Europe. The dimensions of this latter species lie within the range of dimensions of $M$. aestiva $\left(\mathrm{CBL} \sigma^{7} \sigma^{7}\right.$ Coulet des Roches $=45.3 \mathrm{~mm}$ [44.2-46.3 mm] vs recent aestiva $0^{7} \sigma^{7}=47.8 \mathrm{~mm}$ [43.4-50.3] $\mathrm{mm}, n=45$ ). In addition, in this case, similar to the weasel, the smaller size of the stoat confirms the limited role of the ice sheet, which pushed northern species such as the wolverine and the stoat as far south as the Iberian Peninsula $[47,71]$.

Long bones from the smallest mustelids are less informative than the cranial material. However, due to the good state of preservation and their abundance, they can also provide useful information. The analysis of long bones according to their stratigraphic position confirms previous observations (Figure 18). The skeleton associated with the M4 217 skull is particularly small and the long bones are very small, even if they belong to a male. Conversely, the bones found in the clay layer attributed to the climatic oscillation GI 3 and the GI 10-11 interpleniglacial are larger than those from the Pleniglacial, and similar in size to the current common weasel, $M$. nivalis vulgaris. The long bones associated with the ermine skulls are clustered together and are similar in size to present-day weasels. It is important to note that the humerus R 9295 fro the extracted sediments displays similar proportions to those of the weasels from the GI 3 oscillation. 


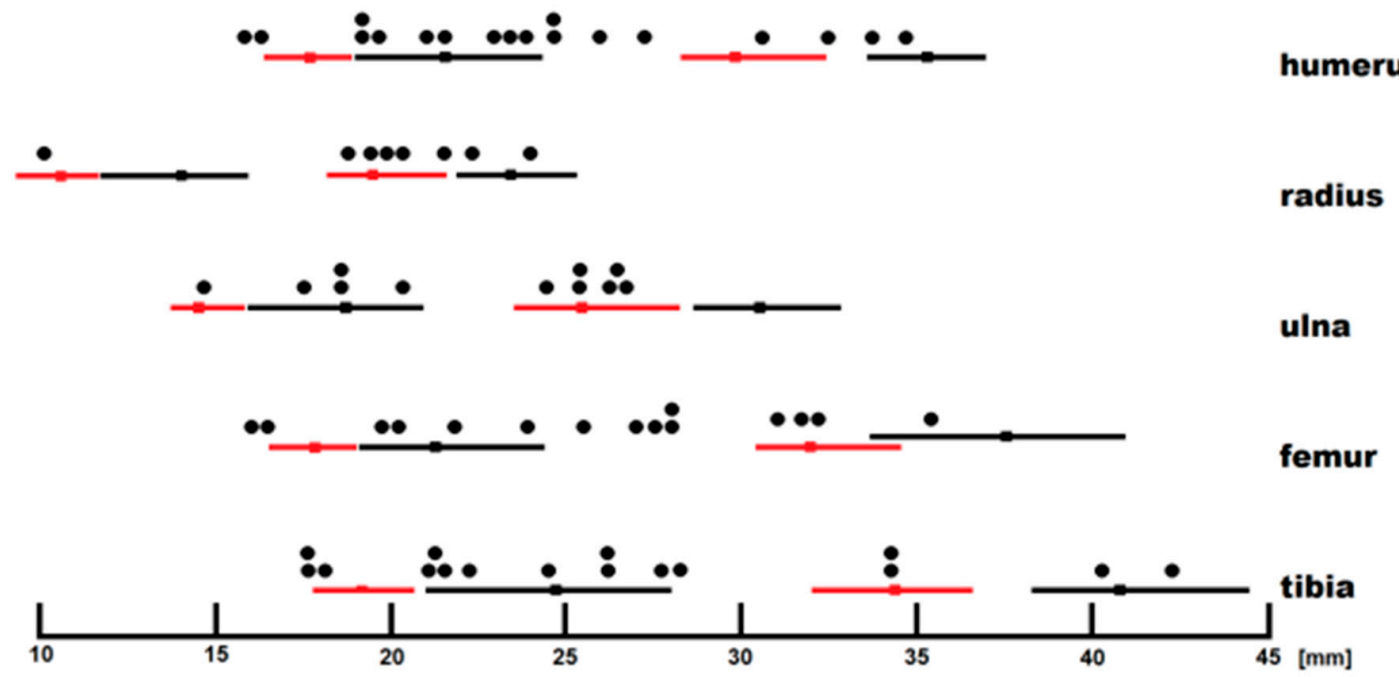

Figure 18. Mustela erminea and Mustela nivalis long bone size comparison (larger, Mustela erminea; smaller, Mustela nivalis; red, males; and black, females). Black circles indicate individuals from Coulet des Roches. Dimensions of comparative recent material according to Rechstein [55,56] and measurements according to A. Marciszak.

\subsubsection{Comments}

The presence of skulls and almost all the bones in a good state of preservation is quite remarkable. The new analysis of the remains led to some adjustments and to more accurate counts of individuals. It confirms the difference in size between weasels and ermines, but also climate-dependent size variations in weasel and ermine individuals. As with many mammals groups, the size of these small mustelids decreased during glaciations.

The presence of the pygmy weasel, M. nivalis minuta, is confirmed throughout LGM and LG (GS 2a). This form is typical of glacial maxima, even in the Iberian Peninsula, and survived in Western Europe in some Alpine and Pyrenean refuge zones during climatic warming [2,62,67]. The M4 217 skeleton is located in the upper part of the glacial unit. At the same depths, the other individuals are larger but are nonetheless smaller than the current $M$. nivalis vulgaris. The few contemporaneous elements from GS 2a are identical in size to the LGM group, with the exception of the individual attributable to the pygmy weasel. The minuta and vulgaris forms thus coexisted in the Coulet des Roches area.

The largest individuals were found in the clay layer deposited during the temperate climatic oscillation GI 3 and during the GI 10-11 interpleniglacial. The metric parameters assimilate them to the current $M$. nivalis vulgaris. The ermine is comparable to the small subspecies M. erminea aestiva, present today in Central Europe, from Russia to Kazakhstan.

\subsection{Equid (NB)}

Equus ferus gallicus Prat, 1968

From 2007 to 2017, 17 horses were identified. Five belong to GS 2a. Six are dated to the end of the LGM, two are contemporaneous with GI 3 and four with the LGM (Table 12). Eleven skulls and nine complete skeletons were preserved (Figures 19 and 20). These horses are similar to the chrono subspecies Equus ferus gallicus [2]. The name Equus ferus is used here and not "caballus", as recommended by the International Commission on Zoological Nomenclature (ICZN) [72], as although the latter name is commonly applied to wild Pleistocene horses, it remains questionable [73,74]. The present analysis concerns the muzzles, the proportion of long bones, the metapodials and the third phalanx, which enable us to evaluate the size of the species and adaptation to climate conditions. 
Table 12. Equus ferus gallicus. Radiocarbon dates. Abbreviation: BA: Beta Analytic.

\begin{tabular}{cccccc}
\hline Calibrated BP & \multicolumn{2}{c}{ Datations IntCal09 } & \multicolumn{3}{c}{ References } \\
\hline IntCal09 & Calibrated BP & Calibrated BC & Material & Sedimentary Unit & Laboratory \\
\hline LG-GS 2a & & & & & \\
18,300-16,300 & $16,450-15,210$ & $14,501-13,261$ & Mare; K4 9 & US3 & BA 267379 \\
& $16,476-15,225$ & $14,528-13,276$ & Mare; N5 76 & US3 & BA 267380 \\
\hline LGM & & & & & \\
& $18,623-18,021$ & $16,623-16,072$ & Mare; K3 111 & US3 & BA 267378 \\
& $21,520-21,320$ & $19,570-19,370$ & Mare; N4 289 & US3 & BA 308066 \\
& $21,670-21,320$ & $19,720-19,370$ & Stallion; M4 547 & US3 & BA 329249 \\
& $24,240-23,950$ & $22,290-22,000$ & Mare; M4 910 & US3 & BA 388485 \\
\hline GI 3 oscillation & & & & & \\
26,000-27,000 & $26,375-26,020$ & $24,425-24,070$ & Stallion; N5 680 & US3 & BA 422567 \\
& $26,700-26,250$ & $24,750-24,300$ & Stallion; L6 41 & US3/clay & BA 422566 \\
\hline LGM & & & & \\
& $27,107-26,610$ & $25,155-24,660$ & Stallion; M5 244 & US3/scree & BA 388486 \\
& $27,462-27,075$ & $25,513-25,126$ & Stallion; M7 97 & US3/scree & BA 476994 \\
& $27,580-27,375$ & $25,630-25,425$ & Mare; L5 78 bis & US3/scree & BA 447017 \\
& $27,783-27,441$ & $25,834-25,492$ & Mare; M6 437-1 & US3/US5 & BA 476993 \\
\hline
\end{tabular}

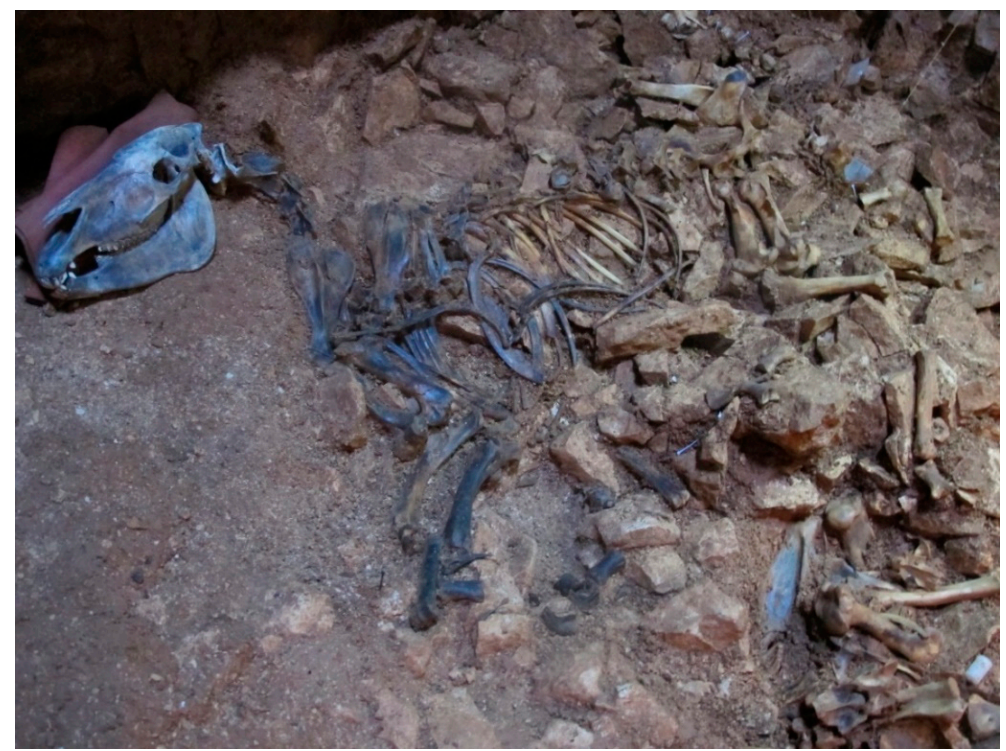

Figure 19. Equus ferus gallicus, Stallion L6 41. Radiocarbon date: $22,190 \pm 90$ cal BP (GI 3). Photo: E. Crégut-Bonnoure.
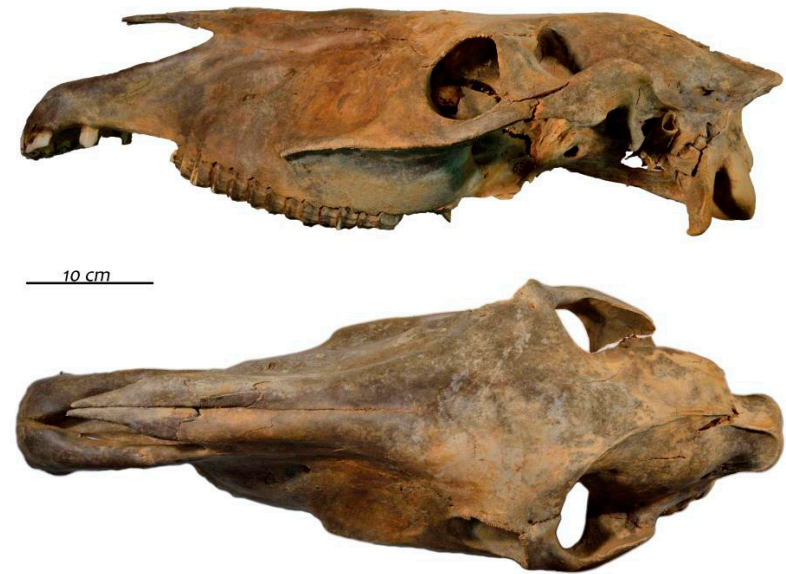

Figure 20. Equus ferus gallicus. Stallion skull L6 41. Radiocarbon date: $22,190 \pm 90$ cal BP (GI3). Photos: C. Triat. 


\subsubsection{Muzzle}

All the individuals, including the new ones, are characterized by a rather short (in comparison to the palatal length, not figured) and large muzzle (Figure 21 and Table 13), as previously shown [2]. The unique specimen from the LG level is in keeping with the distribution of specimens from the LGM (Figure 21). Apart from the long and narrow muzzle of the Portugal horse Equus ferus antunesi, the specimens from Coulet des Roches and other Late Pleistocene horses have a larger muzzle than that of the current Prjewalski's horse (which is just used as a modern comparison sample, as a recent DNA study suggests that this horse may descend from domesticated horses [75]). The three E. ferus gallicus specimens from Jaurens (Corrèze), dating from the end of MIS 3, overlap with those from Coulet des Roches but are on average a little narrower. The two specimens from Siréjol (Gignac, Lot) are clearly narrower. The incisor from the horse from Le Mounoï (Signes, Var) is included in the Coulet des Roches sample (Figure 21). Equus ferus latipes (MIS 2, Mezin, Ukraine) has a longer muzzle and narrower proportions. Finally, the muzzle of the horses from Coulet des Roches is as large but a little longer than the Magdalenian specimen from Le Quéroy (Charente).

The short and large muzzle could represent an adaption to a cold climate and grazer specialization [76-78].

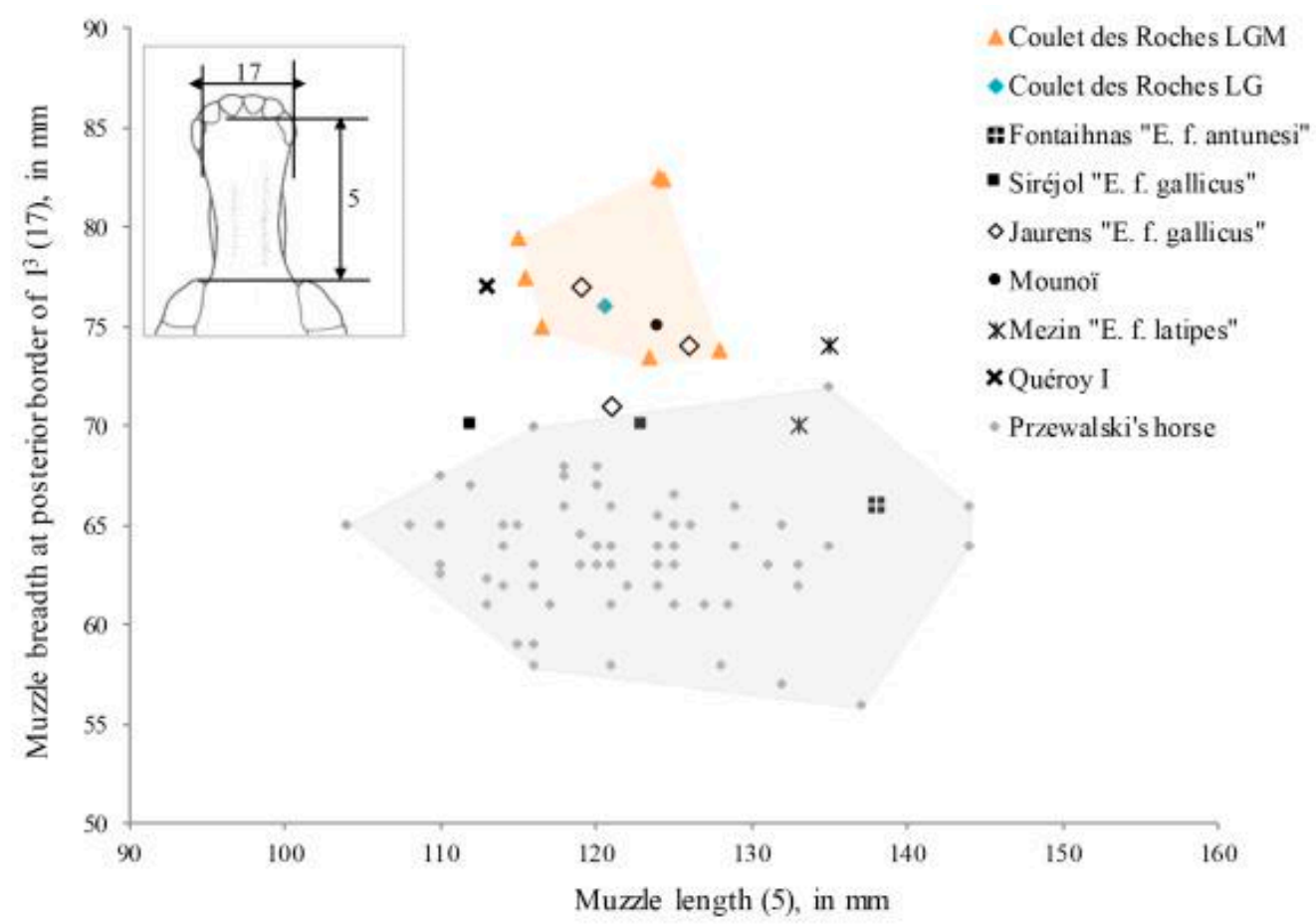

Figure 21. Muzzle length in relation to maximal muzzle breadth. Coulet measurements in Table 13. Comparative data: Przewalski's horse, Siréjol, Jaurens, and Mezin [79]; Fontaihnas [80]; Mounoï [81]; Quéroy I [82]. 
Table 13. Muzzle dimensions of Equus ferus gallicus from Coulet des Roches (in mm). Measurement system according to Eisenmann [79].

\begin{tabular}{cccc}
\hline & & Length (5) & Breadth (17) \\
\hline LG-GS 2a & & & \\
\hline N5-106 & $\wp$ & 120.6 & 76.0 \\
\hline LGM & & & \\
\hline N4.289.1 & $\wp$ & 124.0 & 82.5 \\
M4-546 & $\sigma^{7}$ & 115.0 & 79.5 \\
L4 78 + M4 878 879 & $\wp$ & 116.5 & 75.0 \\
L6-41 & $\sigma^{7}$ & 128.0 & 73.8 \\
N5-680 & $\sigma^{7}$ & & 78.0 \\
L5-78 & $\wp$ & 115.5 & 87.4 \\
M6 437-1 & $\sigma^{7}$ & 124.4 & 73.4 \\
M7 97 & $\sigma^{7}$ & 123.4 & \\
\hline
\end{tabular}

\subsubsection{Limb Bones and the Third Anterior Phalanx}

The differences between Upper Pleistocene Western European horse populations are mainly size related (Figure 22) (Table 14). Horses underwent a decrease in size from the end of the Middle Pleistocene onwards [83-85]. The general size of the horse from Coulet des Roches (LGM and LG) corresponds to the chrono subspecies E. ferus gallicus (end of MIS 3 and MIS 2). Indeed, the average profiles are very close to the type from the "Aurignacian-Perigordian" levels of Solutre (beginning of MIS 2; Saône-et-Loire) and from the population from Jaurens (end of MIS 3) (Figure 22). On the other hand, they are smaller in size than the last E. ferus germanicus ((MIS 3, Tournal B-D (Bize, Aude), Pair-non-Pair (Prignac-et-Marcamps, Gironde)) and larger than E. ferus arcelini (Magdalenian levels of Solutré, end of MIS 2) which is consistent with the absolute dating. The same average size is conserved in the LG sample (Figure 22), while horses are smaller in other regions (Centre of France, the Rhineland), as previously demonstrated by the existence of a geographical cline during this period [85-87]. In Eastern Europe, E. ferus latipes (MIS 2, Mezin, Ukraine) is larger (Figure 22).

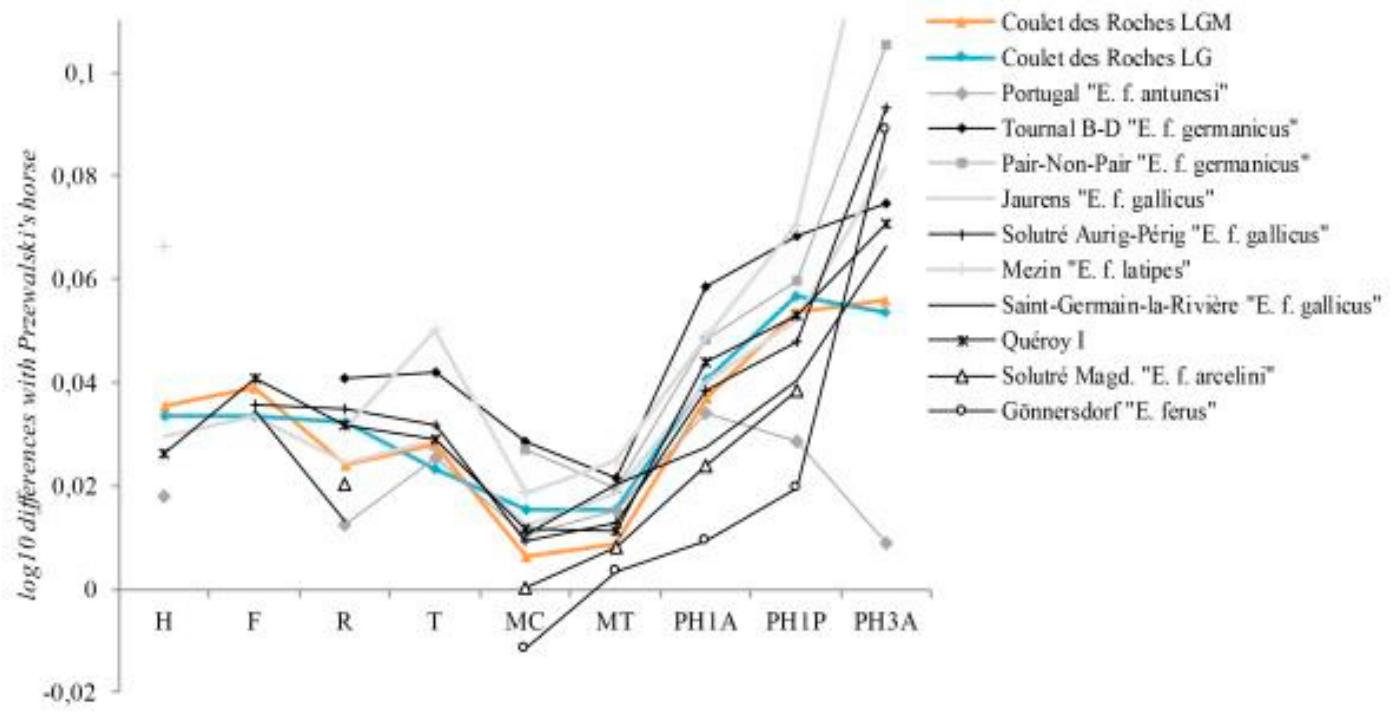

Figure 22. Ratio diagram of bone length and third phalanx breadth of Pleistocene horses. Coulet des Roches measurements in Table 13. Reference: Przewalski's horse [79]. Comparative data: Jaurens and Mezin [79]; Portugal [80]; Tournal B-D [81]; Gönnersdorf [86]; Pair-non-Pair, Saint-Germain-la-Rivière [88]; Solutré [89]; Quéroy I [82]. Abbreviations: H, Humerus; F, Femur; R, Radius; T, Tibia; MC, Metacarpal; MT, Metatarsal; PH1A, First Anterior Phalanx; PH1P, First Posterior Phalanx; PH3A, Third Anterior Phalanx. 
Table 14. Length of the limb bones and third phalanx of Equus ferus gallicus from Coulet des Roches (in $\mathrm{mm}$ ). Average value and number of specimens. Abbreviations: H, Humerus; F: Femur; R, Radius; T, Tibia; MC, Metacarpal III; MT, Metatarsal III; PH1A, First Anterior Phalanx; PH1P, First Posterior Phalanx; PH3A, Third Anterior Phalanx; (), number of specimens.

\begin{tabular}{cccccccccc}
\hline & H & F & R & T & MC & MT & PH1A & PH1P & PH3A \\
\hline LG-GS 2a & $280.9(4)$ & $384.1(6)$ & $332.9(6)$ & $339.3(5)$ & $223.3(6)$ & $266.0(8)$ & $85.7(8)$ & $84.1(8)$ & $79.5(7)$ \\
LGM & $282.2(14)$ & $389.2(11)$ & $326.6(14)$ & $343.3(12)$ & $218.7(19)$ & $261.8(16)$ & $85.1(14)$ & $83.5(13)$ & $80.0(12)$ \\
\hline
\end{tabular}

In terms of proportions, the metacarpal of the Coulet des Roches LGM horse is shorter than that of E. ferus germanicus (Tournal B-D, Pair-non-Pair). This difference may be related to a more open environment during MIS 2. The phalanxes also display differences: the first phalanxes of the Coulet des Roches horses (LGM and LG) are on average as long as those from the beginning of the Upper Paleolithic levels of Solutré (E. ferus gallicus), whereas the first phalanxes of the Magdalenian horses from Saint-Germain-la-Rivière (Gironde), Gönnersdorf (Germany), and to a lesser extent Solutré, are proportionately shorter (Figure 22).

Finally, the third phalanx of the horse from Coulet des Roches is narrower than most other populations of Upper Pleistocene true horses, with the exception of E. ferus antunesi (Figure 22). The horses from Coulet des Roches seem to have frequented less heavy or harder habitat grounds [90].

\subsubsection{Individual Variation of the Metacarpal}

According to the distribution of current equids, the robustness of the metapodial diaphysis correlates well with hygrometric conditions. A more massive diaphysis corresponds to a humid environment and a more gracile diaphysis to a dry/arid environment [91]. Rapid and reversible variations in the proportions of the diaphysis according to this climatic parameter seem to be confirmed on true Pleistocene horses [85]. They are related to phenotypic plasticity rather than a micro-evolutionary mechanism. In the case of Coulet des Roches, slender metapodials could indicate dryer conditions and also geographic particularism [2].

Variations in the proportions of the metacarpal diaphysis at individual level could also reflect climate acclimation. The GS 2a metacarpals are slenderer than those from the LGM (Table 15) (Figure 23). In the sample from the LGM, the GI 3 horses (" $\mathrm{T}^{\prime \prime}$ ) display a more robust metacarpal diaphysis (Figure 23). The robustness index values of these two individuals are close to the average index value of the E. ferus gallicus metacarpals from Solutré (Figure 23), mainly from the formation of "magma" also correlated with the humid oscillation of "Tursac" (GI 3) [89,92,93].

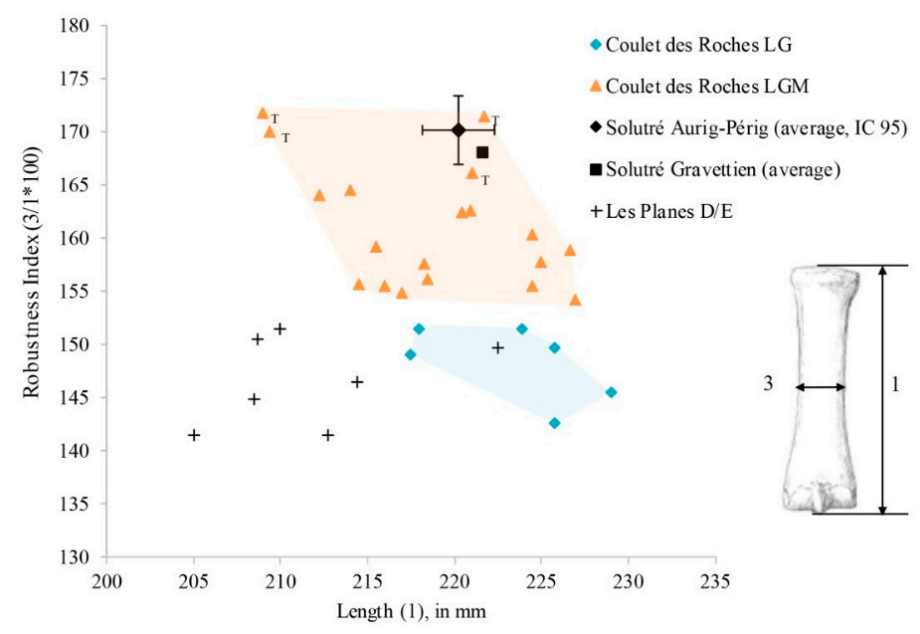

Figure 23. Robustness index in relation to the length of metacarpal III. Robustness index = width of diaphysis in the middle (3)/length $(\mathbf{1}) \times 1000$. Comparative data: Solutré Aurignacian/Perigordian [89]; Solutré Gravettian [86]. Measurement system according to Eisenmann [79]. Abbreviation: T, “Tursac” (GI 3). 
Table 15. Metacarpal III of Equus ferus gallicus from Coulet des Roches (in mm). Abbreviations: L, left; $\mathrm{R}$, right. Measurement system according to Eisenmann [79].

\begin{tabular}{ccccc}
\hline & & Length (1) & Breath at Mid-Diaphysis (3) & 3/1 $\times \mathbf{1 0 0 0}$ \\
\hline LG-GS 2a & & & & \\
\hline M4-133 & L & 218.0 & 33.0 & 151.4 \\
M4-70 & R & 217.5 & 32.4 & 149.0 \\
M4-135 & L & 225.8 & 33.8 & 149.7 \\
L5-4 & R & 223.9 & 33.9 & 151.4 \\
I4-21 & L & 229.0 & 33.3 & 145.4 \\
J4-11 & R & 225.8 & 32.2 & 142.6 \\
\hline LGM & & & & \\
M4-727 & L & 225.0 & 35.5 & 157.8 \\
M4-814 & $\mathrm{R}$ & 224.5 & 34.9 & 155.5 \\
L5-69 & L & 226.9 & 35,0 & 154.3 \\
L5-66 & $\mathrm{R}$ & 226.6 & 36,0 & 158.9 \\
L6-104 & $\mathrm{L}$ & 209.4 & 35.6 & 170.0 \\
L6-96.1 & $\mathrm{R}$ & 209.0 & 35.9 & 171.8 \\
M6-189.1 & $\mathrm{L}$ & 221.0 & 36.7 & 166.1 \\
N6-545 & $\mathrm{R}$ & 221.7 & 38.0 & 171.4 \\
M4-1157 & $\mathrm{L}$ & 216.0 & 33.6 & 155.6 \\
M4-1165 & $\mathrm{R}$ & 214.5 & 33.4 & 155.7 \\
M4-677 & $\mathrm{L}$ & 220.4 & 35.8 & 162.4 \\
N6-291 & $\mathrm{R}$ & 220.9 & 35.9 & 162.5 \\
M4-582 & $\mathrm{L}$ & 212.2 & 34.8 & 164.0 \\
M4-575 & $\mathrm{R}$ & 214.0 & 35.2 & 164.5 \\
M5-477 & $\mathrm{L}$ & 217.0 & 33.6 & 154.8 \\
M5-503 & $\mathrm{R}$ & 215.5 & 34.3 & 159.2 \\
M7-92.1 & $\mathrm{L}$ & 218.4 & 34.1 & 156.1 \\
M6-443.1 & $\mathrm{R}$ & 218.3 & 34.4 & 160.4 \\
M6-405 & $\mathrm{R}$ & 224.5 & 36.0 & \\
\hline & & & & \\
\hline
\end{tabular}

\subsubsection{Discussion}

Regional variation in bone size and proportions occur among LGM and LG equids [85-87,90,94,95]. The horse from Coulet des Roches (LGM and LG) presents anatomical characteristics in keeping with harsh climatic conditions (cool and dry). These differences can be explained by the overall climatic context and specific biogeographic and/or ecological conditions. Due to the very good conservation of the skeletons and the presence of complete individuals, numerous dates, and a paleoclimatic and paleoenvironmental context based on other proxies, the locality of Coulet des Roches could represent a key site for testing the acclimation or adaptation potential of Late Pleistocene true horses and for assessing the validity of the criteria generally used for ecological investigation.

In this sense, the results of the microwear/mesowear signatures of teeth (and in progress by F. Rivals) and further isotopic analysis should be very interesting to "challenge" morphological observations and investigate dietary adaptations, the habitat and environment [96-98].

\subsection{Caprinae (ECB)}

Capra ibex, Linaeus, 1758

Caprinae are represented by the Alpine ibex, Capra ibex. This species is abundant during the Late Pleistocene in the Provence area. Fifteen individuals have been identified: five non-sexed adults, two females, two males, four young, and two juveniles. Ten of them were collected in sediments extracted by spelunkers and are represented by incomplete remains. Five were discovered during the excavation, four of which are represented by complete skeletons: two females, one male and one young (Figure 24). The radiocarbon dates range from the LG to the LGM (Table 16). The spelunkers 
extracted part of the LG sediment, and thus the individuals discovered in this sediment date from this period. Only one of them ( $n^{\circ} \mathrm{R} 6017$; Table 16) was dated by radiocarbon.

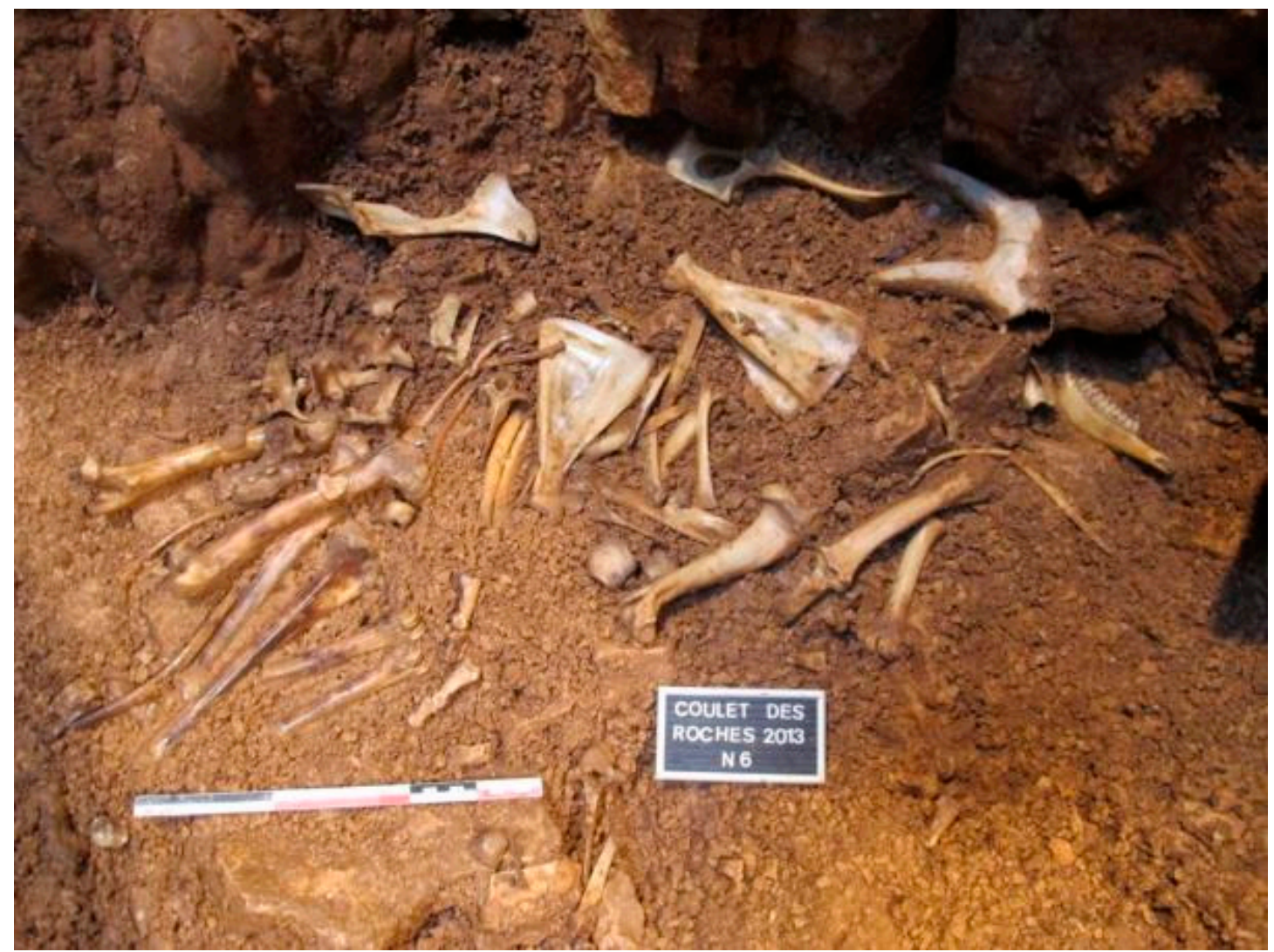

Figure 24. Capra ibex. Female N6 362-1. Radiocarbon date: 18,350 \pm 70 cal BP. Scale: 30 cm. Photo: E. Crégut-Bonnoure.

Table 16. Capra ibex. Radiocarbon dates. Abbreviation: BA, Beta Analytic.

\begin{tabular}{|c|c|c|c|c|c|c|}
\hline \multirow{2}{*}{\multicolumn{2}{|c|}{$\begin{array}{c}\text { Calibrated BP } \\
\text { IntCal09 }\end{array}$}} & \multicolumn{2}{|c|}{ Datations IntCal09 } & \multicolumn{3}{|c|}{ References } \\
\hline & & Calibrated BP & Calibrated BC & Material & Sedimentary Unit & Laboratory \\
\hline \multicolumn{7}{|c|}{ LG } \\
\hline GI 1-e & $14,600-12,900$ & $14,947-14,059$ & $12,998-12,110$ & Young; N3(5) 1 & US3 & BA 267381 \\
\hline GS 2a & $18,300-16,300$ & $16,130-15,220$ & $14,180-13,280$ & Adult; R 6017 & US3 & BA 337294 \\
\hline \multicolumn{7}{|c|}{ LGM } \\
\hline & & $19,382-18,869$ & $17,920-17,432$ & Female; M3 137-2 & US3 & BA 288279 \\
\hline & & $19,560-19,430$ & $17,610-17,480$ & Male; N5 283 & US3 & BA 308067 \\
\hline & & $22,250-21,870$ & $20,300-19,920$ & Female; N6 362-1 & US3 & BA 360688 \\
\hline & & $23,585-23,330$ & $21,635-21,380$ & Young; N5 580 & US3 & BA 388487 \\
\hline
\end{tabular}

The horn core morphology is in accordance with the Alpine ibex (Figure 25). The paleogenetic analysis includes individuals from modern Alpine ibex sequences and French Late Pleistocene samples from Provence: Adaouste (Bouches-du-Rhône; 15,420-15,360 cal BP and 15,260-15,130 cal BP; BA 337,297), Aven Christian (Vaucluse; 12,920-12,690 cal BP; BA 337298), Baume Voutade (Bouches-du-Rhône; 11,610-11,520 cal BP and 11,510-11,290 cal BP; BA 398224) [99,100]. From the LGM to the LG, a modification in tooth morphology is visible: the P/3 metaconid dilates in a small column running from the top to the base of the crown [2]. This morphological transformation was observed in Provence from MIS 3 (Baume Périgaud, Var) to the end of MIS 2 (LG) [101] and in Western Liguria (Grotte du Prince, Grotte des Enfants, Italy) [102]. This transformation is connected to the 
geographic isolation in extant C. pyrenaica from Spain [100,103]. The paleogeography of the Provence area must be taken into consideration to explain this transformation:

- $\quad$ On its eastern side, the area is bordered by the Alps which were covered by giant glaciers during the LGM.

- $\quad$ On its western side, the Rhodanian corridor (Rhône River valley) was not auspicious to exchanges and, up until the end of the Pleistocene, the Durance River, near the Rhodanian corridor, flowed parallel to the Rhône, and directly into the Mediterranean Sea. These two north-south axes formed a double barrier for east-west exchanges [104]. Furthermore, the Durance River, which flowed from east to west before its inflection to the Mediterranean Sea, represented another barrier for the species located on the northern and southern sides.
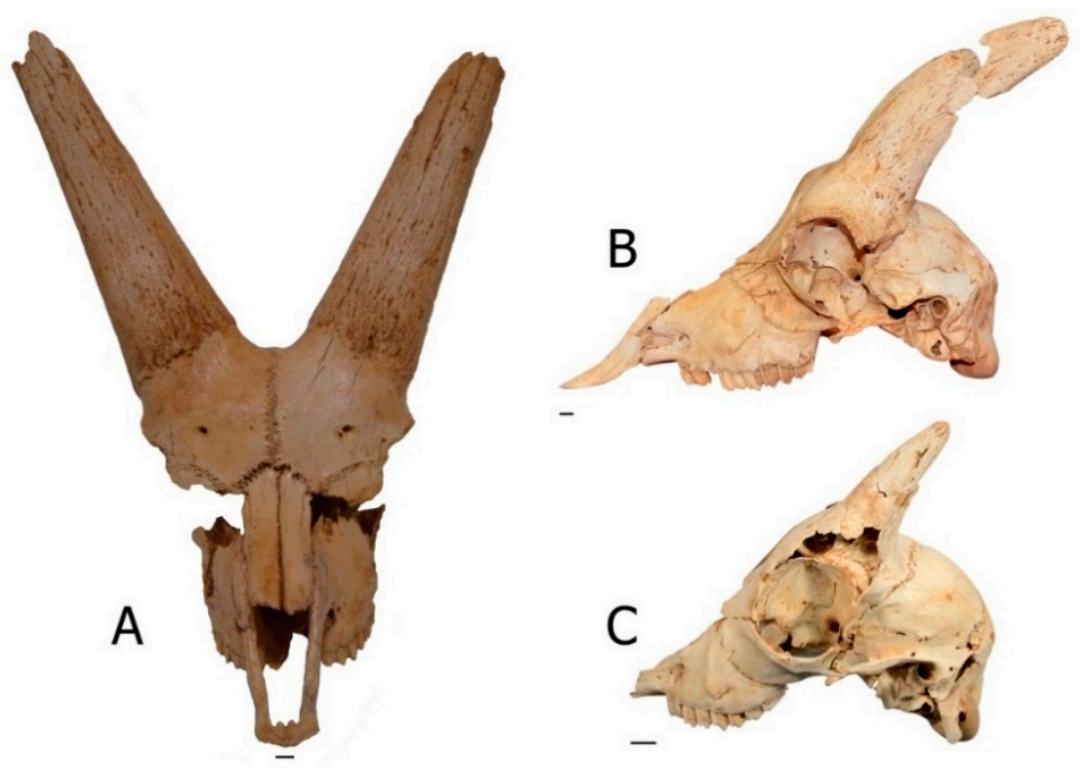

Figure 25. Capra ibex: (A) male N5 283, radiocarbon date: 16,380 \pm 50 cal BP; (B) female N6 362-1, radiocarbon date: $18,350 \pm 70 \mathrm{cal} \mathrm{BP}$; and (C) young N5 580, 8-9 months old, radiocarbon date: 19,460 \pm 60 . Scale: $1 \mathrm{~cm}$. Photos: C. Triat.

For several taxa, such as the ibex, the evident lack of exchange between populations across the Rhône and Durance Rivers led to geographic isolation.

In Coulet des Roches, metaconid dilation is well developed in GS 2a and LGM. However, it is not developed on the LGM P/3 of the female dating from 22,250 to $21,870 \mathrm{cal} \mathrm{BP}$. This could be an individual variation. Another explanation that could be envisaged is that ibex isolation during MIS 3 was more significant on the southern side of the Durance River than on the northern side, which corresponds to the Vaucluse area.

\subsection{Micromammals (ED)}

Excavations carried out at Coulet des Roches yielded abundant small mammal anatomical elements in a well-dated context. It is thus possible to assess the evolution of climatic and environmental changes during the Pleniglacial (and more particularly the LGM/Last Glacial Maximum/HS 2) and also during unstable periods marked by abrupt climatic changes throughout the Late Glacial period (GS 2a/HS 1, GI 1e, and GI 1c-a).

Several hundred anatomical elements have been specifically identified, highlighting the presence of 18 genera and/or species (belonging to 470 individuals, according to the MNI) of insectivores and rodents (Table 17). 
Table 17. Faunal list of the micromammals identified in the Coulet des Roches levels.

\begin{tabular}{|c|c|c|c|c|c|c|c|c|c|c|c|c|c|}
\hline & \multicolumn{7}{|c|}{ Pleniglacial } & \multicolumn{5}{|c|}{ Tardiglacial } & \multirow{3}{*}{$\begin{array}{c}\text { Postglacial } \\
\mathrm{z}=79\end{array}$} \\
\hline & \multicolumn{7}{|c|}{ LGM } & \multicolumn{2}{|c|}{ GS 2a (H1) } & GI 1e & \multicolumn{2}{|c|}{ GI 1ca + GS 1} & \\
\hline & $770<z>660$ & $660<z>600$ & $600<\mathrm{z}<560$ & $560<z<510$ & $510<z<400$ & $400<z<320$ & $320<z<280$ & $280<z<210$ & $180<z<170$ & $160<z<150$ & $120<z<110$ & $110<z<79$ & \\
\hline \multicolumn{14}{|l|}{ Soricomorpha } \\
\hline Talpa europaea & & 1 & 1 & & & & & 2 & & 1 & & & \\
\hline Crocidura leucodon/russula & & & & 1 & & & & & & & & & \\
\hline $\begin{array}{l}\text { cf. Sorex sp. } \\
\text { Sorex minutus }\end{array}$ & & & & & 1 & & & & & 1 & & & \\
\hline $\begin{array}{l}\text { Sorex minutus } \\
\text { Sorex ex gr. araneus/coronatus }\end{array}$ & & & & & $\begin{array}{l}1 \\
1\end{array}$ & & & & & & & & \\
\hline \multicolumn{14}{|l|}{ Rodentia } \\
\hline Marmota marmota & & & 1 & & 2 & & & 1 & 1 & & & & \\
\hline Eliomys quercinus & & & 1 & & 1 & & & 1 & & 1 & & 2 & \\
\hline Apodemus sylvaticus & 2 & 3 & 7 & 5 & 3 & & & & & 3 & 1 & 6 & 1 \\
\hline Arvicola amphibius & & & & & 1 & & & & & 1 & & & \\
\hline Microtus (Stenocranius) gregalis & & 2 & & & & & & & & & & & \\
\hline Microtus arvalis & 6 & 20 & 35 & 14 & 19 & 6 & 2 & & 1 & 1 & & & \\
\hline Microtus ex gr. arvalis/agrestis & & & & & & & & 5 & 1 & 5 & 3 & 3 & 1 \\
\hline Chionomys nivalis & 11 & 34 & 78 & 35 & 42 & 13 & 3 & 3 & & 1 & 3 & 1 & 1 \\
\hline Clethrionomys glareolus & & 1 & & & 2 & 1 & & 1 & & 2 & & & \\
\hline Microtus (T.) duodecimcostatus & & & & & & & & & & 1 & & & \\
\hline Microtus (T.) cf. multiplex & & & & & 1 & & & & & 1 & & & \\
\hline Microtus oeconomus & & 6 & 3 & 8 & 3 & 5 & & & 1 & & & & \\
\hline Dicrostonyx torquatus & & 2 & 5 & 2 & 20 & 2 & & 1 & & & & & \\
\hline Total MNI & 19 & 69 & 131 & 65 & 96 & 27 & 5 & 14 & 4 & 18 & 7 & 12 & 3 \\
\hline
\end{tabular}

Abbreviations: $\mathrm{z}$, altitudinal level; MNI, minimum number of individuals. 
Rodent associations are mostly characterized by a species assigned to the Arctic (Dicrostonyx torquatus) or boreal (Microtus oeconomus) parts of Northern Europe or encountered in regions with a highly contrasted continental climate (Chionomys nivalis and Marmota marmota). Among the rodent species, the presence of the collared lemming $(D$. torquatus) is particularly interesting. Lemmings are currently represented by 12 species, all of which are confined to Arctic regions and adapted to a cold climate and arid environments. Excavations at Coulet des Roches yielded more than $60 \mathrm{~m} 1$ relating to the Dicrostonyx genus (Figure 26). This constitutes the most abundant collection of lemmings collected in the Pleistocene in southeastern France. It should be noted that the $\mathrm{m} 1$ of the Coulet des Roches collared lemming presents high variability, in terms of both morphology and size. However, according to the size of the $\mathrm{m} 1$, the Coulet des Roches lemmings represent a single species: D. torquatus. In Southern Europe, this species has been found in a few prehistoric sites dating from the Upper Paleolithic (Late Upper Pleistocene). According to the numerous absolute dates of the different levels and large mammal associations identified at Coulet des Roches, the presence of $D$. torquatus in the lower and upper levels of the site confirms that this species was widely distributed in south-eastern France during the LGM and GS 2a (Figure 27). Although it is difficult to reason in terms of absence, it seems that the GS 2a was the last phase of expansion of this species in Mediterranean regions.
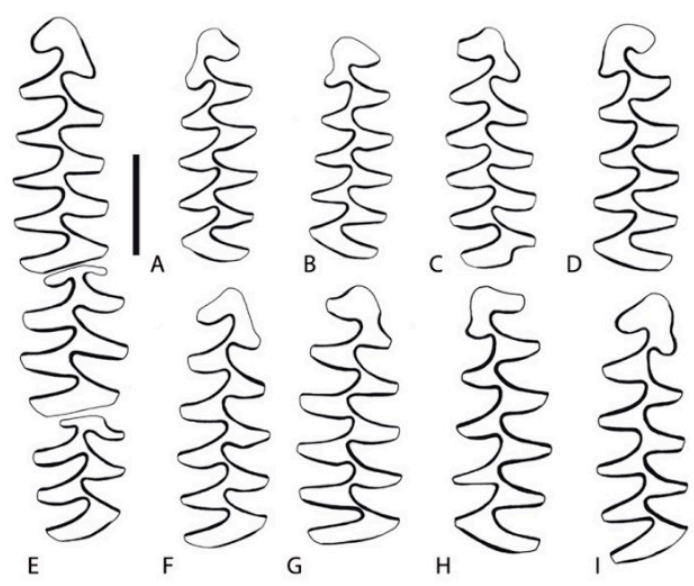

Figure 26. Dicrostonyx torquatus of Coulet des Roches: (A) M4 R788 z $=4.36 \mathrm{~m} 1$ left; (B) M5 R34 z $=-4.60 \mathrm{~m} 1$ left; (C) M4 R493 z = -4.36 m1 right; (D) N6 R249 z = -4.50 m1 1 left; (E) M4 R957 $\mathrm{z}=-4.45 \mathrm{~m} 1-\mathrm{m} 3$ right; (F) M4 R468 $\mathrm{z}=-4.47 \mathrm{M} / 1$ right; (G) M4 R493 $\mathrm{z}=-4.36 \mathrm{~m} 1$ right; (H) N5-N6 R170 $\mathrm{z}=-4.85 \mathrm{~m} 1$ left; and (I) M5 R34 $\mathrm{z}=-4.60 \mathrm{~m} 1$ right. Scale: $1 \mathrm{~mm}$. Abbreviation: $\mathrm{z}$, altitudinal level.

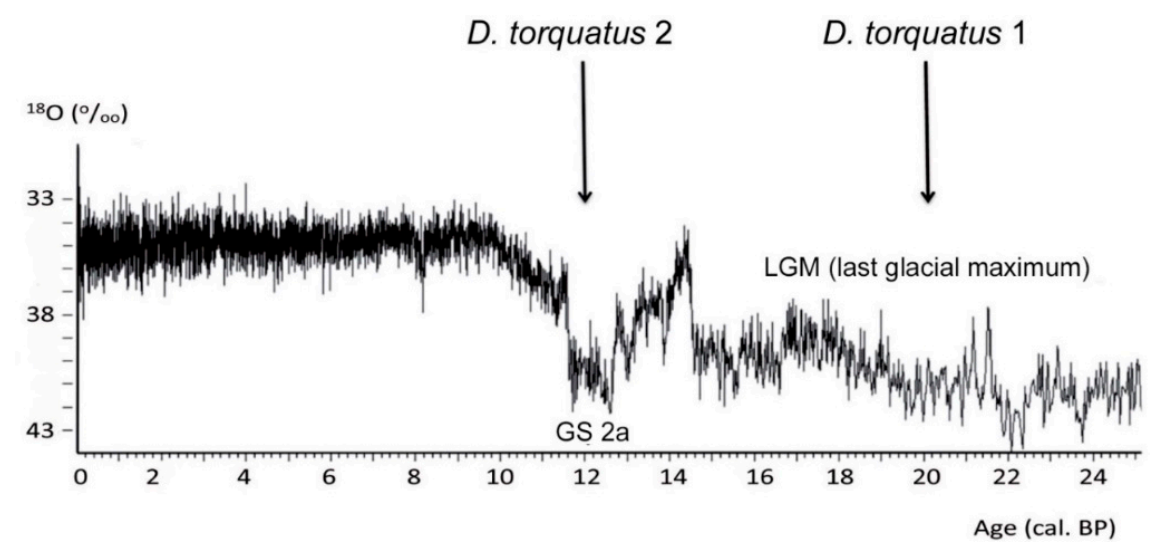

Figure 27. Chronology of the last expansions of D. torquatus at the end of the Upper Pleistocene. 


\subsubsection{Taphonomy}

Large and small fossil mammal faunas are not an exact reflection of the original faunas as many biases may have distorted the image of the initial wildlife spectrum. It is therefore necessary to understand and interpret the different processes involved after the death of the animal and its burial in the sediments, as these processes can alter our perception and the reality of the past. Indeed, taphonomic biases are numerous, complex, and closely related to the environment in which living communities have been incorporated. Multiple factors are likely to occur, such as exposure to climate, predators, differential conservation, trampling, chemical modifications of soils, transport, diagenesis or the reworking of stratigraphic levels. For this reason, we considered it to be appropriate to first carry out the reconstruction of the taphonomic history of the micromammal assemblages from Coulet des Roches, following the standard methodology described in previous taphonomic studies (e.g., [105-117]), which includes skeletal element representation, fragmentation patterns and analysis of surface modifications of bones and teeth (especially digestion). The accumulation of micromammals at Coulet des Roches probably corresponds to a coprocoenosis of nocturnal raptors (Figure 28), possibly by the snowy owl (Bubo scandiacus) [118]. Another predator, probably a carnivore, may also be responsible for part of the micromammal accumulation, as evidenced by traces of digestion on the bone material. This predator is both eclectic and opportunistic. Therefore, its hunting habits are not likely to induce a significant representation bias and should be representative of the fauna surrounding Coulet des Roches. Conversely, some carnivores likely to have accumulated part of the assemblage-M. nivalis, for example—have a more specialized diet [119-121]. However, this possible predation bias must be very limited in the case of the LGM at Coulet des Roches as only a small proportion of the assemblage is likely to be linked to a coprocoenosis induced by such specialized carnivores.

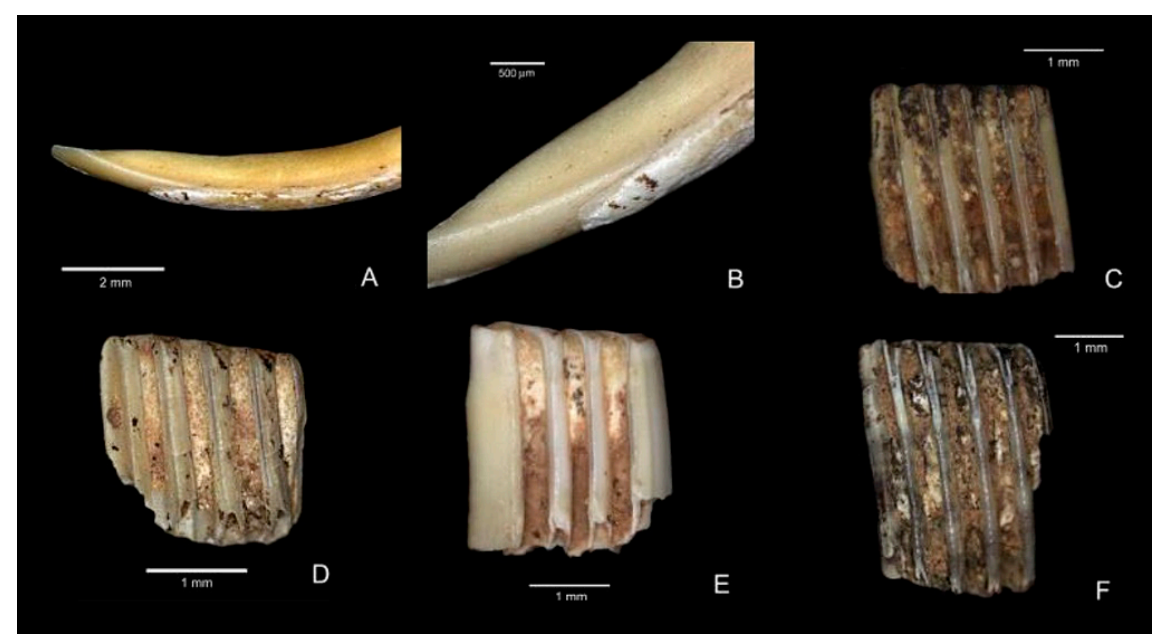

Figure 28. Taphonomy: rodent teeth displaying digestion induced by avian raptors. Photos: E. Desclaux and L. Woodthorpe.

\subsubsection{Paleoecology}

From the base to the top of the sequence, micromammal communities are not homogeneous and reveal variations in paleoenvironments that may be related to known paleoclimatic oscillations during the Pleniglacial (LGM) and the Late Glacial period (GS 2a/HS 1, GI 1e, and GI 1c-a).

Meticulous sieving of the soil yielded a significant number of dental remains in sediments at the known base of the sequence, thus enriching the micromammal record and providing unpublished paleontological data concerning Pleniglacial climatic and environmental variations in Southern France. 


\section{Climatogram Method}

Rodent faunas, which are relatively abundant at the base of the sequence, can be assigned to specific biotopes to establish a "climatogram", according to the quantitative method developed by Chaline [122]. It therefore seemed interesting to group rodent species into climato-ecological categories by considering current and fossil associations. We used current data relating to the biology, climate, vegetation and habitat of the species identified in the sequence (principle of actualism) and the ecological groups defined by Chaline $[123,124]$. We also considered the paleoecological data obtained through the use of multivariate analyses applied to rodent associations by Marquet [125], Desclaux [126] and Foury et al. [127].

The rodents of Coulet des Roches were grouped into eight climato-ecological categories:

Category I: cold arctic steppes (ARC): D. torquatus

Category II: forested and humid boreal areas (BOR): M. oeconomus.

Category III: continental steppes (OPEN): Microtus (Stenocranius) gregalis, Microtus arvalis.

Category IV: open mountain areas (MOUNT): M. marmota, Ch. nivalis.

Category V: riverbanks (WAT): Arvicola sapidus.

Category VI: meadows (HUM): Microtus (Terricola) multiplex, Microtus agrestis.

Category VII: deciduous forests (DECID): Apodemus sylvaticus, Eliomys quercinus, Myoxus glis, Chletrionomys glareolus.

Category VIII: Mediterranean region (MED): Terricola (Terricola) duodecimcostatus.

The climatogram provides interesting data concerning the evolution of climate dynamics and environments from the LGM (Figure 29) until the end of the Late Glacial (Figure 30).

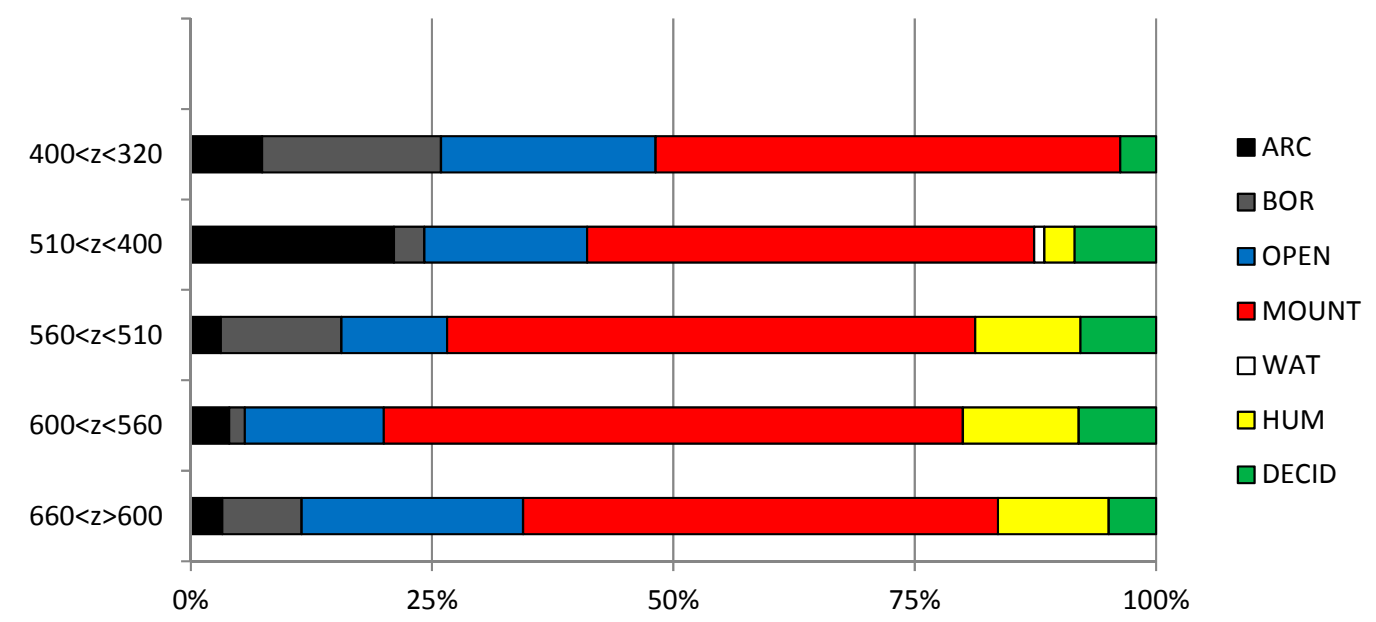

Figure 29. Climatogram of the LGM based on the MNI of rodent species identified in the Coulet des Roches levels. ARC, cold arctic steppes; BOR, forested and humid boreal areas; OPEN, continental steppes; MOUNT, open mountain areas; WAT, riverbanks; HUM, meadows; DECID, deciduous forests; MED, Mediterranean region.

At the known base of the infill $(660<\mathrm{z}<600)$, we note the presence of $D$. torquatus, M. oeconomus and M. (Stenocranius) gregalis; three species assigned to the northernmost areas of Europe. Twenty individuals are attributed to D. torquatus-which is extremely rare in Southern Europe-showing that this configuration is representative of the coldest periods of the Pleistocene. The hypothesis of an attribution of the known base of the filling of Coulet des Roches to the LGM is therefore very plausible. In addition, the overall proportion of species characteristic of a cold climate and an open environment increases from the base to the top of the lower part of the LGM $(660<\mathrm{z}<400)$, to the detriment of more humid and closed environments and rather temperate climatic conditions. This trend indicates that the cold optimum is reached at $510<\mathrm{z}<400$. 


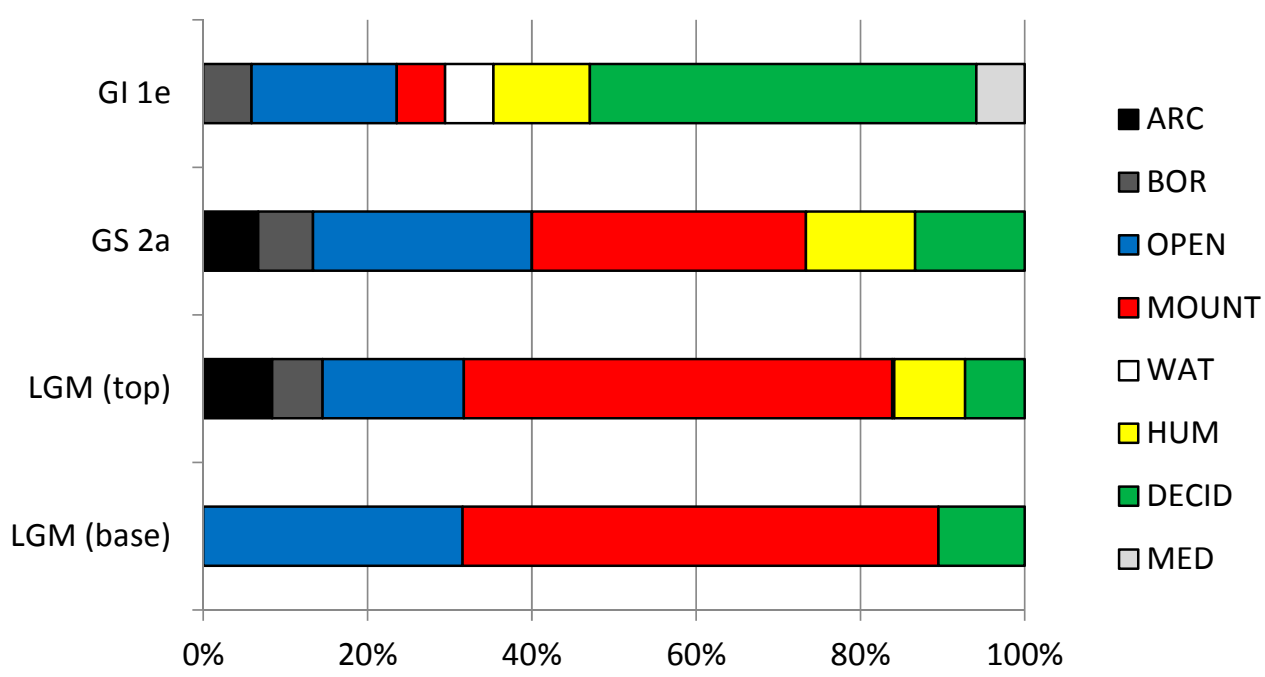

Figure 30. Climatogram of all the levels of Coulet des Roches based on the MNI of the identified rodent species.

The overlying part of the filling $(280<\mathrm{z}<160)$ is characterized by the persistence of $D$. torquatus and M. marmota. This association is representative of an open environment (steppe) and a particularly cold climate. The open aspect of the environment is however moderated by the presence of $A$. sylvaticus and $\mathrm{Cl}$. glareolus; two mostly forested species. This part of the filling is therefore likely to date from GS $2 \mathrm{a}$, which seems to correspond to the last expansion of the collared lemming in Southern France.

The upper levels $(160<\mathrm{z}<150)$ show a greater variety of rodent species. The association of E. quercinus, A. sylvaticus and $\mathrm{Cl}$. glareolus is characteristic of a forested environment. The presence of M. (Terricola) duodecimcostatus and M. (Terricola) cf. multiplex highlights the humid character of the climate. This level is likely to date from the GI 1-e.

Finally, the stratigraphy ends with less abundant and diversified rodent communities $(120<\mathrm{z}<110)$, characterized by a decline in species inhabiting closed environments and a humid climate, in favor of species inhabiting open areas under a continental climate ( $\mathrm{Ch}$. nivalis and M. marmota). These levels can be attributed to GS 1 .

The Taxonomic Habitat Index (THI)

It also seemed appropriate to use the Taxonomic Habitat Index (THI), first introduced by Nesbit-Evans et al. [128]. This consists in calculating the cumulative index by combining the presence/absence and the habitat indications of all the species in a faunal community, notably micromammals [113]. Basically, the THI is a method of combining information for each taxon in a composite paleoenvironmental interpretation, regardless of the relative proportions of species identified in a site.

The base of the infill $(170<\mathrm{z}<620)$, which includes the LGM $(280<\mathrm{z}<620)$ and the GS $2 \mathrm{a}$ $(170<\mathrm{z}<280)$, is characterized by a clear representation of the northern environments (tundra and taiga) and continental areas (Alpine regions). The persistence of forested environments (deciduous forests) and Mediterranean biotopes highlights the southern position of the site and the fact that the Mediterranean biome served as a refuge area during the cold episodes of the Upper Pleistocene, which further explains the high micromammal diversity observed at the base of the sequence (Figure 31). 


\begin{tabular}{|l|c|c|c|c|c|c|c|c|}
\cline { 2 - 8 } \multicolumn{1}{c|}{} & $\mathrm{Tu}$ & $\mathrm{B}$ & $\mathrm{A}$ & $\mathrm{S}$ & $\mathrm{Fs}$ & $\mathrm{Mo}$ & $\mathrm{D}$ & $\mathrm{Med}$ \\
\hline Talpa europaea & 0.00 & 0.20 & 0.00 & 0.10 & 0.10 & 0.00 & 0.50 & 0.10 \\
Crocidura leucodon-russula & 0.00 & 0.00 & 0.20 & 0.20 & 0.10 & 0.30 & 0.20 & 0.00 \\
Sorex araneus & 0.20 & 0.20 & 0.05 & 0.05 & 0.10 & 0.30 & 0.10 & 0.00 \\
Sorex minutus & 0.30 & 0.30 & 0.00 & 0.00 & 0.00 & 0.05 & 0.30 & 0.05 \\
Dicrostonyx torquatus & 1.00 & 0.00 & 0.00 & 0.00 & 0.00 & 0.00 & 0.00 & 0.00 \\
Microtus oeconomus & 0.40 & 0.50 & 0.00 & 0.00 & 0.10 & 0.00 & 0.00 & 0.00 \\
Microtus (Stenocranius) gregalis & 0.45 & 0.00 & 0.20 & 0.35 & 0.00 & 0.00 & 0.00 & 0.00 \\
Clethrionomys glareolus & 0.00 & 0.40 & 0.00 & 0.00 & 0.10 & 0.10 & 0.40 & 0.00 \\
Apodemus sylvaticus & 0.00 & 0.00 & 0.20 & 0.20 & 0.20 & 0.00 & 0.20 & 0.20 \\
Eliomys quercinus & 0.00 & 0.00 & 0.00 & 0.00 & 0.00 & 0.00 & 0.40 & 0.60 \\
Microtus arvalis & 0.00 & 0.00 & 0.50 & 0.10 & 0.10 & 0.00 & 0.30 & 0.00 \\
Microtus agrestis & 0.00 & 0.00 & 0.00 & 0.00 & 0.20 & 0.00 & 0.50 & 0.30 \\
Arvicola amphibius & 0.10 & 0.15 & 0.00 & 0.05 & 0.05 & 0.02 & 0.35 & 0.28 \\
Microtus (Terricola) duodecimcostatus & 0.00 & 0.00 & 0.15 & 0.00 & 0.15 & 0.00 & 0.20 & 0.50 \\
Microtus (Terricola) multiplex & 0.00 & 0.00 & 0.00 & 0.00 & 0.10 & 0.50 & 0.30 & 0.10 \\
Chionomys nivalis & 0.00 & 0.00 & 0.20 & 0.10 & 0.00 & 0.40 & 0.10 & 0.20 \\
Marmota marmota & 0.00 & 0.00 & 0.20 & 0.40 & 0.20 & 0.20 & 0.00 & 0.00 \\
Lepus timidus & 0.20 & 0.20 & 0.00 & 0.20 & 0.20 & 0.20 & 0.00 & 0.00 \\
Oryctolagus cuniculus & 0.00 & 0.00 & 0.20 & 0.00 & 0.20 & 0.00 & 0.20 & 0.40 \\
\hline
\end{tabular}

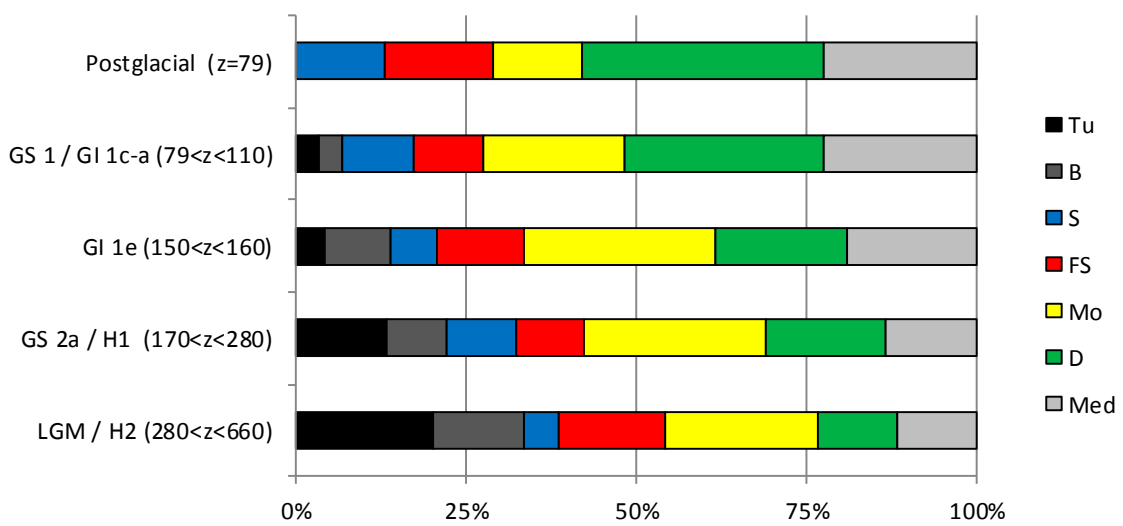

Figure 31. Taxonomic Habitat Index (THI) values observed at Coulet des Roches. Tu, tundra; B, boreal forest; A, arid area; S, steppe; Fs, forest-steppe; Mo, mountain; D, deciduous forest; Med, Mediterranean biome.

The overlying part of the sequence-which might concern GI 1-e $(150<\mathrm{z}<160)$ and GI 1c-a/GS 1 $(79<\mathrm{z}<120)$-is characterized by a less conspicuous presence of species that currently inhabit the northern regions of Europe.

The main contribution of the THI method is to emphasize the southern location of Coulet des Roches by very clearly highlighting the persistence of typical Mediterranean species during the LGM and to show the persistence of northern taxa (such as Lepus timidus) during the more temperate episodes (during GI 1-e and GI 1c-a, for example). 


\section{Cenogram Method}

The cenogram method was developed by Valverde [129] and slightly modified by Legendre $[130,131]$ for application to fossil faunas. It is based on the principle that particular aspects of the weight distribution of mammal species can be related to certain environmental characteristics. It presents the advantage of allowing for the reconstitution of the milieu by considering all mammals (except chiropters and carnivores, regardless of their relative proportions). This method has already been applied to the Quaternary faunas of southeastern France by Desclaux $[132,133]$ and Montuire and Desclaux $[134,135]$.

Cenograms were only developed for the LGM, GS 2a and GI 1e levels of Coulet des Roches, as these levels contain enough large mammal species (Figure 32). The cenograms are characterized by a large slope for large mammals—due to low species diversity in the site-and discontinuity between large and small species. Overall, this weight distribution corresponds to an arid and open environment.

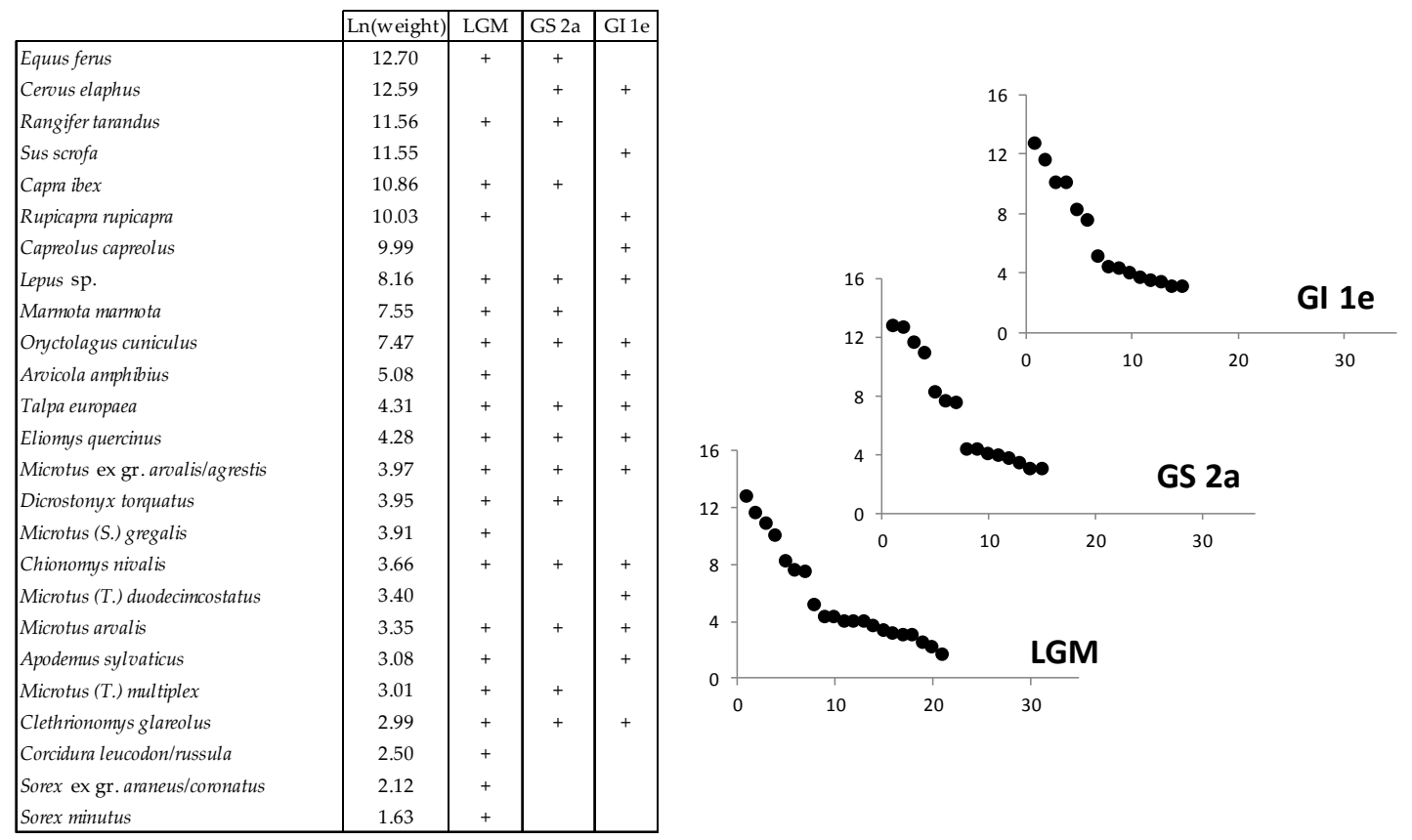

Figure 32. Cenograms of LGM, GS 2a and GI 1e levels of Coulet des Roches, showing an open and arid environment. Ln(weight), Neperian logarithm of weight.

However, smaller mammals, which are much more diversified, moderate the coldness of the climate, highlighting the southern position of the site and thus suggesting the persistence of Mediterranean species in the region, including during the colder stages of the Upper Pleistocene.

The fact that the GI 1e cenogram is similar in character to GS 2a and LGM may be due to the fact that the collected fauna (large and small mammals) are only a partial reflection of the surrounding mammalian diversity. It is therefore best not to take this cenogram into account.

\section{The Non-Analog Communities of Micromammals}

It is important to recall that the Vaucluse is a complex and interesting region in terms of the succession of associations of small and large mammals:

- Lowland areas, particularly along the Rhône-which were a corridor and shelter for temperate species during glacial episodes-may be related to the classical southern refuges for the Late Pleistocene in the Mediterranean region [136-139]. According to climatic fluctuations, these plains allowed for the passage of temperate species to Provence ("southern refuge zone", in the 
classical sense of the term) during cold stages and their re-immigration to Western Europe during interglacial periods.

- Highland areas, in the north and east of the area, in the foothills of the Alpine Massif, not only played a natural role as a geographical barrier, but also formed a refuge zone during interglacial episodes for certain northern and eastern species ("cryptic southern refugia", according to the terminology used by Stewart et al. [140] and in accordance with the observations of Schmidt [141]).

This geographical configuration probably favored the presence of so-called "no-analog" communities of small mammals, composed of currently unrecognized sympatric species in the Coulet des Roches filling. It explains why D. torquatus is found associated with A. sylvaticus, M. (T.) multiplex and Ch. nivalis during the LGM and E. quercinus and Ch. nivalis during the GS 2a.

Various species with distinct climatic-ecological affinities could therefore transition from the LGM to GI 1c-a in the Vaucluse, as evidenced by the presence of so-called "no-analog" micromammal communities in the Coulet de Roches infilling. Such associations suggest that two types of contiguous refuges coexisted in the region during MIS 2. Only two millennia separate the LGM from the LG (GS 2a). It is therefore possible that, during the LGM, species originating from northern (D. torquatus and M. oeconomus) and eastern (Marmota marmota) parts of Europe, with considerable episodic expansion to Southern Europe during the cold periods of the Pleistocene, were able to find a refugium in the Alpine mountains at the end of the LGM. The Alps are therefore likely to have played the role of a refugium ("cryptic southern refugia") for certain species dependent on harsh and continental climates. This configuration would have facilitated the sporadic incursion of these three species in the Mediterranean biome, during the cold climatic oscillation of GS 2a.

\section{Comments}

The abundant and well-preserved material of two small mustelids, the stoat and weasel, allows for a detailed metric and morphological analysis, as well as the evaluation of size changes throughout the course of time under specific climatic conditions. The most considerable differences were found in M. nivalis, where two different ecotypes are represented. Specimens from the cold period (LGM) are the typical representative of the highly specialized pygmy weasel, which is indistinguishable from the recent dwarf forms from the minuta-rixosa group. These small weasels were later (in the LG and GI 3 levels) replaced by moderate-sized, more opportunistic weasels from the nivalis-vulgaris group, forms that are recently widespread in Europe. The M. erminea material is more comparable with the medium-sized subspecies M. erminea aestiva, which mainly currently occurs in Central Europe. However, the LGM specimens are smaller than the specimens from the GI 3 humid oscillation. The variability in the stoat and weasel dimensions from Coulet des Roches shows that both small mustelids do not follow Bergmann's rule. Additionally, the third mustelid, the common polecat M. putorius, was found in the LGM and GS 2a sediments from Coulet des Roches. This species is associated with mosaic and forest habitats, usually near water reservoirs (streams and ponds). The examined individuals do not differ in size and morphology from the modern European common polecat.

The conservation of the horse remains is exceptional. All the specimens belong to E. ferus gallicus. The variation between individuals is low and their characteristics point to harsh climatic conditions (cool and dry), except for the two individuals dating from the humid oscillation GI 3, with a robust third metacarpal, similar to the Solutré population from the same oscillation.

The ibex is characteristic of LGM populations from the Provence area, with a p3 metaconid developed in a column. However, diachronism is observed in comparison with the population located on the southern side of the Durance River. In Coulet des Roches, located on the northern side of the Durance River, the metaconid dilation of the oldest LGM individual is missing, whereas it is well expressed on the southern side before the LGM. This raises the question of the role of the Durance as a geographical barrier. 
The abundant micromammals enable us to follow the evolution of the specific associations in the studied area (Vaucluse). This evolution is complex and interesting during the period from the LGM to the beginning of the LG (GS 2a) climate warming. Paleoecological analyses of the abundant and diversified micromammal communities record the climatic and environmental variations from the Pleniglacial to the Tardiglacial. The presence of so-called "no-analog" communities of small mammals suggests that, during MIS 2, two types of contiguous refuges coexisted in the region. It is therefore possible that, during the LGM, species originating from northern (D. torquatus and M. oeconomus) and eastern (M. marmota) parts of Europe, with sporadic expansion to Southern Europe during the cold periods of the Pleistocene, were able to find a refugium in the Alpine mountains at the end of the LGM. The Alps are therefore likely to have played a refugium role ("cryptic southern refugia") for certain species dependent on harsh and continental climates. This would have facilitated the episodic incursion of these three species into the Mediterranean biome, during the GS 2a cold climatic oscillation.

\section{Conclusions}

The faunal assemblage from Coulet des Roches is unprecedented for Southern France and the infilling provides information spanning a period of 21,000 years, from the Subboreal to the LGM, and even from the GI 3, perhaps the GI 4 and GI 10-11 oscillations. Among the fauna, the cold and dry climate guild from units LG-GS 2a and LGM comprises Alopex lagopus, M. nivalis minuta, M. erminea aestiva, Rangifer tarandus, Lepus timidus, M. oeconemus, D. torquatus and Bubo scandiacus. This guild occurs for the first time in the Provence area. Numerous ongoing studies on some species (Ch. nivalis, lagomorphs, birds, and microwear-mesowear of ungulate teeth) will complete our knowledge of the LG and LGM fauna.

The present analysis of mustelids, equids, ibexes and micromammal, provides new information on the variation of species from the LG and LGM in relation to the environment. Some of these species reflect ecological adaptation and provide new evidence of the phenotypic plasticity of the taxa. The micromammal analysis raises the question of the refugium role played by Southern France under cold climates.

In addition to the interest of this vertebrate accumulation for paleontology (morphometric characterization and adaptive responses in relation to the climate) and paleoecology (phenotypic expressions depending on the climate and variation of the association in relation to the climate), the exhumed remains are now a vital reference for paleogenetic studies. They are also a reference for the characterization of natural traps, the conditions associated with their sedimentary fillings and taphonomic processes.

Author Contributions: Investigation, E.C.-B., N.B., E.D., and A.M. Supervision: E.C.-B.

Funding: The excavations were financially supported by D.R.A.C-P.A.C.A., Avignon municipality, Calvet Foundation, Association of the Friends of Requien Museum, Association for the Promotion of Mediterranean Prehistory and Anthropology, and Archaeological department from Vaucluse.

Acknowledgments: We wish to thank the Malavard family, the owners of Coulet des Roches, for their authorization to excavate and their kindness towards us. We also thank the Sault municipality and L'Hôte family for providing accommodation. Special thanks to Joël Mathieu for his logistic support and to the Association des Amis de Saint-Jean-de Sault for their welcome and assistance. We thank our colleague Jacqueline Argant for fruitful discussions on the chronology of the LG and LGM. We thank Maxime Pelletier for providing unpublished data concerning leporids. The excavation was carried out in close collaboration with the Departmental Committee of Speleology from Vaucluse (CDS 84), the Vauclusian Speleological clubs from Carpentras, Avignon (SSA and AVEN), Bollène, Camaret, Cavaillon and Courthézon, the Ariégeois Speleological clubs from Arize, Cousserans and the Tarn Speleological club of Sorrèze. We would like to thank all the spelunkers who oversaw the speleological aspect of this excavation, especially Michel Baillet, Christian Bérard, Gilles Druart, Jean-Paul Granier, Alain Gruneisen and Robert Russ. Special thanks to Cédric Triat from Muséum Requien for the photos used here. Sincere thanks to the anonymous reviewers for their very useful comments and suggestions which greatly improved the text.

Conflicts of Interest: The authors declare no conflict of interest. 


\section{References}

1. Shackleton, N.J. The oxygen isotope stratigraphic record oi the late Pleistocene. Philos. Trans. R. Soc. 1977, 280, 169-179. [CrossRef]

2. Crégut-Bonnoure, E.; Argant, J.; Bailon, S.; Boulbes, N.; Bouville, C.; Buisson-Catil, J.; Debard, E.; Desclaux, E.; Fietzke, J.; Fourvel, J.-B.; et al. The karst of the Vaucluse, an exceptional record for the Last Glacial Maximum (LGM) and the Late-glacial period palaeoenvironment of southeastern France. Quat. Int. 2014, 339-340, 41-61. [CrossRef]

3. Daniel, M. Et la taupe y creusa son trou. Blousons d'Argile 1979, 9, 45-48.

4. Jean, M. Le Coulet des Roches $\mathrm{n}^{\circ}$ 1. Blousons d'Argile 1973, 5, 6.

5. Jean, M. Activités sportives. Blousons d'Argile 1977, 8, 6-7.

6. Le Fahler, B.; Perret, J.-F. Aven du Coulet des Roches $\mathrm{n}^{\circ} 1$ ou du Petit cheval. In Les Arcanes de Vaucluse. Hydrogéologie et Spéléologie des Territoires Alimentant en Eau la Fontaine de Vaucluse; Gaubert, G., Le Fahler, B., Eds.; Association de Recherches et d'Etudes Hydrogéologiques du Plateau d'Albion: Avignon, France, 1995; Volume 2, p. 223.

7. Parein, R. Guide Spéléo des Monts de Vaucluse; Compte d'auteur: Cavaillon, France, 1990; 96p.

8. Parein, R.; Languille, A. La Haute-Provence Souterraine; Compte d'auteur: Cavaillon, France, 1981; 422p.

9. Crégut-Bonnoure, E. Monieux: Coulet des Roches. Scientific Report of the PACA Region; Archeological Regional Service: Aix-en-Provence, France, 2007; pp. 245-246. (In French)

10. Crégut-Bonnoure, E. Monieux: Coulet des Roches. Scientific Report of the PACA Region; Archeological Regional Service: Aix-en-Provence, France, 2008; pp. 223-224. (In French)

11. Crégut-Bonnoure, E. Monieux: Coulet des Roches. Scientific Report of the PACA Region; Archeological Regional Service: Aix-en-Provence, France, 2009; pp. 239-241. (In French)

12. Crégut-Bonnoure, E. Monieux: Coulet des Roches. Scientific Report of the PACA Region; Archeological Regional Service: Aix-en-Provence, France, 2010; pp. 227-228. (In French)

13. Crégut-Bonnoure, E.; Boulbes, N.; Desclaux, E. Monieux: Coulet des Roches. Scientific Report of the PACA Region; Archeological Regional Service: Aix-en-Provence, France, 2014; pp. 214-215. (In French)

14. Crégut-Bonnoure, E.; Desclaux, E.; Frèrebeau, N.; Marciszak, A.; Roger, T. Monieux: Coulet des Roches. Scientific Report of the PACA Region; Archeological Regional Service: Aix-en-Provence, France, 2012; pp. 217-218. (In French)

15. Crégut-Bonnoure, E.; Boulbes, N.; Fourvel, J.-B.; Frèrebeau, N. Monieux: Coulet des Roches. Scientific Report of the PACA Region; Archeological Regional Service: Aix-en-Provence, France, 2013; pp. 204-205. (In French)

16. Argant, J.; Boulbes, N.; Crégut-Bonnoure, E.; Desclaux, E.; Pelletier, M.; Rivals, F. Monieux: Coulet des Roches. Scientific Report of the PACA Region; Archeological Regional Service: Aix-en-Provence, France, 2016; pp. 195-196. (In French)

17. Crégut-Bonnoure, E.; Boulbes, N.; Desclaux, E.; Cohen, J.; Jamieson, A.; Nadachoswki, A.; Roger, T.; Woodthorpe, L. Coulet des Roches (Monieux, Vaucluse); Progress Report of Excavation in the PACA Region; Archeological Regional Service: Aix-en-Provence, France, 2017; 195p. (In French)

18. Rodríguez Varela, R.; Tagliacozzo, A.; Ureña, I.; García, N.; Crégut-Bonnoure, E.; Mannino, M.A.; Arsuaga, J.L.; Valdiosera, C. Ancient DNA evidence of Iberian lynx palaeoendemism. Quat. Sci. Rev. 2015, 112, 172-180. [CrossRef]

19. Reimer, P.J.; Baillie, M.G.L.; Bard, E.; Bayliss, A.; Beck, J.W.; Blackwell, P.G.; Bronk Ramsey, C.; Buck, C.E.; Burr, G.S.; Edwards, R.L.; et al. IntCal09 and Marine09 radiocarbon age calibration curves, 0-50,000 years cal BP. Radiocarbon 2009, 51, 1111-1150. [CrossRef]

20. Anderson, E. Quaternary evolution of the Genus Martes (Carnivora, Mustelidae). Acta Zool. Fenn. 1970, 130, 1-132.

21. Marciszak, A. Ssaki Łasicowate (Mustelidae, Carnivora, Mammalia) z Plejstocenu Polsk. Ph.D. Thesis, Zakład Paleozoologii Uniwersytetu Wrocławskiego, Wrocław, Poland, 2012.

22. Wurm, A. Über eine neuentdeckte Steppenfauna von Mauer an der Elsenz (bei Heidelberg). Jahresber. Mitt. Oberrh. Geol. Ver. 1913, 3, 62-78.

23. Kormos, T. Zur Frage des Mitteleuropäischen Pleistozän-Iltis. Mitt. Jahrb. Ung. Geol. Reichsanst. 1916, 23, 459-476.

24. Soergel, W. Der Steppeniltis, Foetorius Eversmanii Less. aus dem oberen Travertinen des Travertingebietes von Weimar. Zeitschrift der Deutschen Geologischen Gesellschaft 1917, 69, 139-181. 
25. Ehik, G. Der Steppeniltis (Mustela eversmanii Less.) in Ungarn. Annales Historico-Naturales Musei Nationalis Hungarici 1928, 25, 1-38.

26. Kretzoi, M. Tigeriltis, Iltis und Nerz im ungarischen Pleistozän. Földtani Közlöny 1942, 72, 323-344.

27. Koby, F.E. Le Putois d'Eversmann fossile en Suisse et en France. Le Putois d'Eversmann Fossile en Suisse et en France 1951, 44, 394-398.

28. Heller, F. Die Fauna. Das Paläolithikum in den Weinberghöhlen bei Mauern. Quartär 1955, 2, $220-307$.

29. Malez, M. Kvartarna fauna Pecine Veternice u Medvednici. Palaeontol. Jugosl. 1963, 5, 1-193.

30. Heptner, W.G. Über die morphologischen und geographischen Beziehungen zwischen Mustela putorius und Mustela eversmanii. Zeitschrift für Säugetierekunde 1964, 29, 321-330.

31. Zapfe, H. Die übrigen Carnivoren (außer Höhlenhyäne und Höhlenbär). Denkschr. Österr. Akad. Wiss. Math.-Naturwiss. Kl. 1966, 112, 23-38.

32. Heptner, V.G.; Naumov, N.P. Mlekopitayushchije Sovetskogo Soyuza; Part lb, Żinszczinyje (Kunicy; Dopolnitelnych Widow); Vysshaya Shkola Publishers: Moscow, Russia, 1967; Volume II.

33. Sickenberg, O. Der Steppeniltis (Mustela (Putorius) eversmanii soergeli Ehik) in der Niederterrasse der Leine und seine klimageschichtliche Bedeutung. Eiszeitalt. Ggw. 1968, 19, 147-163.

34. Delpech, F. Présence de «Mustela minuta» Pomel (1853) (= «Mustela rixosa» Bangs, 1896) dans les couches aurignaco-périgordiennes du gisement de Roc de Combe (commune de Payrignac, Lot). Bull. Assoc. Fr. Quat. 1973, 10, 3-6. [CrossRef]

35. Hugueney, M. Les mustelidés (Mammalia, Carnivora) du gisement pléistocène moyen de la Fage (Corrèze). Arch. Mus. Hist. Nat. Lyon 1975, 13, 29-46.

36. Clot, A. La grotte de la Carrière (Gerde, Hautes-Pyrénées). Stratigraphie et paléontologie des Carnivores. Travaux Laboratoire Géologie Toulouse 1980, 3, 1-502.

37. Gautier, A. Notes sur les mammifères. In La Caverne Marie-Jeanne (Hastière-Lavaux, Belgique). Mém. Musée Hist. Nat. Belgique 1980, 177, 25-42.

38. Crégut-Bonnoure, E.; Guérin, C. Le gisement pléistocène de l'aven de la Terrasse à Aiguèze (Gard). Première découverte dans le Midi méditerranéen de Mustela (Putorius) eversmanii (Mammalia, Carnivora). Bull. Soc. Étude Sci. Nat. Vaucluse 1982, 1, 135-164.

39. Wolsan, M. Mustela putorius. In Handbuch der Säugetiere Europas. Band 5: Raubsäuger-Carnivora (Fissipedia). Teil II: Mustelidae 2, Viverridae, Herpestidae, Felidae; Niethammer, J., Krapp, F., Eds.; AULA-Verlag: Wiesbaden, Germany, 1993; pp. 699-769.

40. Wolsan, M. Mustela eversmanii. In Handbuch der Säugetiere Europas. Band 5: Raubsäuger-Carnivora (Fissipedia). Teil II: Mustelidae 2, Viverridae, Herpestidae, Felidae; Niethammer, J., Krapp, F., Eds.; AULA-Verlag: Wiesbaden, Germany, 1993; pp. 770-816.

41. Tournepiche, J.-F. Les grands mammifères pléistocènes de Poitou-Charentes. Paleo 1996, 8, $109-141$. [CrossRef]

42. Mallye, J.-B.; Guérin, C. Le gisement Pléistocène superieur de la Grotte de Jaurens à Nespouls, Corrèze, France: les Mustelidae (Mammalia, Carnivora). Cahiers Scientifiques du Muséum d'histoire Naturelle de Lyon 2002, 1, 15-53.

43. Fosse, P.; Fourvel, J.B. Les mustelidés de Romain-la-Roche (Doubs, France). Rev. Paleobiol. 2010, $29,603-611$.

44. Galik, A. Jungpleistozäne Ungulaten und Musteliden (Mammalia) Aus der Schusterlucke im Kremstal (Waldviertel). Master's Thesis, Veterinare und Medizine Universitüt Wien, Vienna, Austria, 1996.

45. Galik, A. Die pleistozänen Iltisknochen (Mustelidae, Mammalia) aus der Schusterlucke im Kremstal (Waldviertel, Niederösterreich): Mustela putorius L.-Mustela eversmanii LESS. Wissensch. Mitt. Niederösterr. Landesmuseum 1997, 10, 45-61.

46. Novikov, G.A. Chiscnye Mlekopitajuscije Fauny SSSR; Izdatelstwo Nauk SSSR: Moskva, Russia, 1956.

47. Altuna, J. Fauna de mamíferos de los yacimientos prehistóricos de Guipúzcoa. Munibe 1971, 24, 1-464.

48. Stresemann, E. Exkursionsfauna für die Gebiete der DDR und BRD. Teil 3: Wirbeltiere; Volk und Wissen Verlang: Berlin, Germany, 1974.

49. Heller, F. Die Höhlenruine Hunas bei Hartmannshof (Landkreis Nürnberger Land)-eine paläontologische und urgeschichtliche Fundstelle aus dem Spät-Riß. Quartär 1983, 4, 1-407.

50. Buchalczyk, T. Rząd: Drapieżne-Carnivora. In Klucz do Oznaczania Ssaków Polski; Pucek, Z., Ed.; Państwowe Wydawnictwo Naukowe: Warszawa, Poland, 1984; pp. 256-310. 
51. Baryshnikov, G.F.; Golovanova, L.V. On the Mousterian site Matuzka in the Kuban Caucasus. Proc. Zool. Inst. 1989, 198, 1-55.

52. Delpech, F. Les Mustelidés. In La Baume de Gigny (Jura); Campy, M., Chaline, J., Vuillemey, M., Eds.; CNRS: Paris, France, 1989; pp. 61-68.

53. Dufour, R. Les Carnivores pléistocènes de la caverne de Malarnaud (Ariège) (Collection, E. Harlé, Muséum d'Histoire Naturelle de Bordeaux), DES de Sciences Naturelles; Université Bordeaux: Bordeaux, France, 1989.

54. David, A.I.; Tatarinov, K.A.; Svistun, W.I. Żinszcznyje, chobotnyje i kopytnyje rannego plejstocenajugo-zapada SSSR; Sztinca: Kisziniev, Moldova, 1990.

55. Reichstein, H. Mustela erminea. In Handbuch der Säugetiere Europas. Band 5: Raubsäuger-Carnivora (Fissipedia). Teil II: Mustelidae 2, Viverridae, Herpestidae, Felidae; Niethammer, J., Krapp, F., Eds.; AULA-Verlag: Wiesbaden, Germany, 1993; pp. 533-571.

56. Reichstein, H. Mustela nivalis. In Handbuch der Säugetiere Europas. Band 5: Raubsäuger-Carnivora (Fissipedia). Teil II: Mustelidae 2, Viverridae, Herpestidae, Felidae; Niethammer, J., Krapp, F., Eds.; AULA-Verlag: Wiesbaden, Germany, 1993; pp. 571-627.

57. Garcia, N.G. Osos y otros Carnívoros de la Sierra de Atapuerca; Fundación Oso de Asturias: Madrid, Spain, 2003.

58. Baryshnikov, G.F. Pleistocene Mustelidae (Carnivora) from Paleolithic site in Kudaro Caves in the Caucasus. Russ. J. Theriol. 2009, 8, 75-95. [CrossRef]

59. Sommer, R.; Benecke, N. Late- and post-glacial history of the Mustelidae in Europe. Mamm. Rev. 2004, 34, $249-284$. [CrossRef]

60. Wojtal, P. Zooarchaeological Studies of the Late Pleistocene Sites in Poland; Institute of Systematics and Evolution of Animals, Polish Academy of Sciences: Kraków, Poland, 2007.

61. King, C.M. The Advantages and Disadvantages of Small Size to Weasels, Mustela Species. Carnivore Behavior, Ecology E Evolution; Gittleman, J., Ed.; Cornell University Press: London, UK, 1989; pp. 302-334.

62. King, C.M.; Powell, R.A. The Natural History of Weasels and Stoats. Ecology, Behavior, and Management; Oxford University Press: New York, NY, USA, 2007.

63. Jędrzejewska, B.; Jędrzejewski, W. Ekologia Zwierząt Drapieżnych Puszczy Białowieskiej; Polskie Wydawnictwa Naukowe: Warszawa, Poland, 2001.

64. Š́álek, M.; Spassov, N.; Anděra, M.; Enzinger, K.; Ottlecz, B.; Hegyeli, Z. Population status, habitat associations, and distribution of the steppe polecat Mustela eversmanii in Europe. Acta Theriol. 2013, 58, 233-244. [CrossRef]

65. Hernández Fernández, M. Análisis Paleoecológico y Paleoclimático de las Sucesiones de Mamíferos del Plio-Pleistoceno Ibérico. Ph.D. Thesis, Universidad Complutense de Madrid, Madrid, Spain, 2001.

66. Crégut-Bonnoure, E. 18,000 ans d'évolution de la faune mammalienne en Vaucluse. Bull. Archéol. Provence 2008, 5-6, 45-60.

67. Abramov, A.V.; Baryshnikov, G.F. Geographic variation and intraspecific taxonomy of weasel Mustela nivalis (Carnivora, Mustelidae). Zoosyst. Rossica 2000, 8, 365-402.

68. Dayan, T.; Tchernov, E. On the first occurrence of the common weasel (Mustela nivalis) in the fossil record of Israel. Mammalia 1988, 52, 165-168. [CrossRef]

69. Clot, A.; Brochet, G.; Chaline, J.; Desse, G.; Evin, J.; Granier, J.; Mein, P.; Mourer-Chauviré, C.; Omnes, J.; Rage, J.C. Faune de la grotte préhistorique du Bois du Cantet (Espèche, Hautes-Pyrénées, France). Munibe 1984, 36, 33-50.

70. Kratochvil, J. Studies on Mustela erminea (Mustelidae, Mamm.) L. variability of metric and mass traits. Folia Zool. 1977, 26, 291-304.

71. Döppes, D. Gulo gulo (Mustelidae, Mammalia) im Jungpleistozän Mitteleuropas. Beitr. Paläontol. 2001, 26, 1-95.

72. International Commission on Zoological Nomeclature (ICZN). Opinion 2027 (Case 3010). Usage for 17 specific names based on wild species which are pre-dated by or contemporary with those based on domestic animals (Lepidoptera, Osteichtyes, Mammalia): Conserved. Bull. Zool. Nomencl. 2003, 60, 801-884.

73. Eisenmann, V. Discriminating Equus skulls: The Franck's index and the new palatal index. In Equids in Time and Space, Proceedings of the 9th ICAZ Conference, Durham, UK, 23-28 August 2002; Mashhour, M., Ed.; Oxbow Books: Barnsley, UK, 2006; pp. 172-182.

74. Guadelli, J.-L.; Delpech, F. La faune des niveaux inféreurs de la grotte Temnata Dupka. In Temnata Cave, Excavations in Karlukovo Karst Area; Ginter, B., Kozlowski, J.K., Guadelli, J.-L., Sirakova, S., Eds.; Jagellonian University Press: Krakow, Poland, 2000; Volume 3, pp. 31-72. 
75. Gaunitz, C.; Fages, A.; Hanghøj, K.; Albrechtsen, A.; Khan, N.; Schubert, M.; Seguin-Orlando, A.; Owens, I.J.; Bignon-Lau, O.; de Barros Damgaard, P.; et al. Ancient genomes revisit the ancestry of domestic Przewalski's horses. Science 2018, 360, 111-114. [CrossRef] [PubMed]

76. Eisenmann, V.; Crégut-Bonnoure, E.; Moigne, A.-M. Equus mosbachensis et les grands Chevaux de la Caune de l'Arago et de Lunel-Viel: Crâniologie comparée. Bull. Mus. Natl. Hist. Nat. 1985, 7, 157-173.

77. Caloi, L. The Two Equids of the Middle Pleistocene of the site Venosa-Loreto (Southern Italy): Functional morphology of the cranial remains. Geol. Romana 2002, 36, 275-287.

78. Bignon, O. Diversité et exploitation des Équidés au Tardiglaciaire en Europe occidentale. Implications pour les stratégies de subsistance et les modes de vie au Magdalénien et à l'Azilien Ancien du Bassin de Paris. Ph.D. Thesis, Université Paris X-Nanterre, Paris, France, 2003.

79. Cardoso, J.L.; Eisenmann, V. Equus caballus antunesi, nouvelle sous-espèce quaternaire du Portugal. Palaeovertebrata 1989, 19, 47-72.

80. Eisenmann, V. Vera Eisenmann. Available online: http:/ / www.vera-eisenmann.com/ (accessed on 9 November 2017).

81. Boulbes, N. Metric datas of the horses from Tournal (Bize-Minervois, Aude) and Le Monoï (Signes, Var). Unpublished work.

82. Eisenmann, V.; Metric datas of the horse from Le Quéroy (Chazelles, Charente). Personal communication, 2017.

83. Forsten, A. Size decrease in Late Pleistocene-Holocene caballoid horses (Genus Equus) intra- or interspecific evolution? A discussion of alternatives. Quat. Int. 1993, 19, 71-75. [CrossRef]

84. Eisenmann, V.; David, F. Évolution de la taille des chevaux d'Arcy-sur-Cure et de quelques autres chevaux quaternaires dans l'Aurignacien de la Grotte du Renne. In Gallia Préhistoire; Schmider, B., Ed.; Éditions du CNRS: Paris, France, 2002; pp. 97-102.

85. Magniez, P.; Boulbes, N.; Brugal, J.P. Variations de la taille corporelle chez les ongulés pléistocènes: Implications paléoécologiques et taphonomiques. In Taphonomies; Brugal, J.-P., Ed.; Archives Contemporaines: Paris, France, 2017.

86. Cramer, B. Morphometrische Untersuchungen an Quartären Pferden in Mitteleuropa. Ph.D. Thesis, Eberhard Karls Universität Tübingen, Tübingen, Germany, 2002.

87. Valensi, P.; Boulbes, N. «La faune magdalénienne du Roc-aux-Sorciers (Angles-sur-l’Anglin, Vienne), Collection Suzanne de Saint-Mathurin». In Angles-Sur-l'Anglin (Vienne). L'abri-Sous-Roche du Roc-Aux-Sorciers, Ses Occupants les Magdaléniens et Leur Rapport à L'art; Pinçon, G., Ed.; Comité des travaux historiques et scientifiques: Paris, France, in preparation.

88. Prat, F. Recherche sur les Equidés Pléistocènes en France. Ph.D. Thesis, Faculty of Sciences, University of Bordeaux, Bordeaux, France, 1968.

89. Guadelli, J.L. Les chevaux de Solutré (Saône et Loire, France). Cahier du Quaternaire 1991, 16, 261-336.

90. Bignon, O.; Eisenmann, V. Western European Late Glacial horses diversity and its ecological implications. In Equids in Time and Space, Proceedings of the 9th ICAZ Conference, Durham, UK, 23-28 August 2002; Mashkour, M., Ed.; Oxbow Books: Barnsley, UK, 2006; pp. 161-171.

91. Eisenmann, V. Sur quelques caractères adaptatifs du squelette d'Equus et leurs implications paléoécologiques. Bull. Mus. Natl. Hist. Nat. 1984, 2, 185-195.

92. Argant, A.; Argant, J. (Eds.) Difficultés et enseignements de l'analyse palynologique appliquée à la préhistoire: Étude du site de Solutré (Saône-et-Loire). In Palynologie et Archéologie; Notes et Monographies Techniques. Actes des Journées du 25-26-27 janvier; CNRS: Paris, France, 1984; pp. 163-181.

93. Combier, J.; Montet-White, A. Solutré 1968-1998; MSPF: Paris, France, 2002; 281p.

94. Kaagan, L.M. The Horse in Late Pleistocene and Holocene Britain. Ph.D. Thesis, University of London, London, UK, 2000.

95. Bignon, O.; Baylac, M.; Vigne, J.D.; Eisenmann, V. Geometric morphometrics and the population diversity of Late Glacial horses in Western Europe (Equus caballus arcelini): Phylogeographic and anthropological implications. J. Archaeol. Sci. 2005, 32, 375-391. [CrossRef]

96. Rivals, F.; Julien, M.A.; Kuitems, M.; Van Kolfschoten, T.; Serangeli, J.; Drucker, D.G.; Bocherens, H.; Conard, N.J. Investigation of equid paleodiet from Schoningen 13 II-4 through dental wear and isotopic analyses: Archaeological implications. J. Hum. Evol. 2015, 89, 129-137. [CrossRef] [PubMed]

97. Daujeard, C.; Vettese, D.; Béarez, P.; Boulbes, N.; Britton, K.; Crégut-Bonnoure, E.; Desclaux, E.; Lateur, N.; Pike-Tay, A.; Rivals, F.; et al. Neanderthal selective hunting of reindeer? The case study of Abri du Maras 
(south-eastern France). In Archaeological and Anthropological Sciences; Springer: New York, NY, USA, 2017; pp. 1-27.

98. Uzunidis, A.; Rivals, F.; Brugal, J.-P. Relation between morphology and dietary traits in horse jugal upper teeth during the Middle Pleistocene in Southern France. Quaternaire 2017, 28, 303-312. [CrossRef]

99. Ureña, I.; Ersmark, E.; Galindo-Pellicena, M.A.; Crégut-Bonnoure, E.; Samaniego, J.A.; Olivencia, A.; Rios-Garaizar, J.; Garate, D.; Gilbert, M.T.P.; Arsuaga, J.L.; et al. Unraveling the history of the European wild goats. Quat. Sci. Rev. 2017, 185, 189-198. [CrossRef]

100. Crégut-Bonnoure, E. Les Ovibovini, Caprini et Ovini (Mammalia, Artiodactyla, Bovidae, Caprinae) du Plio-Pléistocène d'Europe: Systématique, Évolution et Biochronologie. Ph.D. Thesis, Université de Lyon I, Villeurbanne, France, 2002.

101. Crégut-Bonnoure, E. Intérêt biostratigraphique de la morphologie dentaire de Capra (Mammalia, Bovidae). Ann. Zool. Fenn. 1991, 28, 273-290.

102. Moussous, A. Approche Biostratigraphique et Évolution des Paléoenvironnements au Cours du Pléistocène Supérieur, D’après L'étude des Grands Mammifères des Grottes de Balzi Rossi (Ligurie, Italie). Ph.D. Thesis, Université de Perpignan Via Domitia, Perpignan, France, 2014.

103. Crégut-Bonnoure, E. Biochronologie et grands Mammifères au Pléistocène moyen et supérieur en Europe occidentale: L'apport des genres Hemitragus et Capra. Quaternaire 2009, 20, 481-508. [CrossRef]

104. Monjuvent, G.; Aloïsi, J.-C.; Ambert, P.; Bonifay, E.; Bonnet, A.; Campy, M.; Clauzon, G.; Colomb, E.; Dorthe-Monachon, C.; Dubar, M.; et al. Interglaciaires, paléogéographie. In Synthèse Géologique du Sud-Est de la France; Debrand-Passard, S., Ed.; BRGM: Paris, France, 1984; p. 126.

105. Voorhies, M. Taphonomy and population dynamics of an early Pleistocene vertebrate fauna, Know County, Nebraska. Univ. Wyo. Contrib. Geol. Spec. Pap. 1969, 1, 1-69.

106. Dodson, P. The significance of small bones in palaecological interpretation. Contrib. Geol. 1973, 12, $15-19$.

107. Duke, G.; Jegers, A.; Loff, G.; Evanson, A. Gastric digestion in some raptors. Comp. Biochem. Physiol. 1975, 50, 649-656. [CrossRef]

108. Dodson, P.; Wexlar, D. Taphonomic Investigations of Owl Pellets. Paleobiology 1979, 5, 275-284. [CrossRef]

109. Behrensmeyer, A. The taphonomy and paleoecology of Plio-Pleistocene vertebrate assemblages east of Lake Rudolf, Kenya. Bull. Mus. Comp. Zool. 1975, 146, 473-578.

110. Behrensmeyer, A.; Hill, A. Fossils in the Making. Vertebrate Taphonomy and Paleoecology; Prehistoric Archeology and Ecology Series; University of Chicago Press: Chicago, IL, USA, 1980; 345p.

111. Lyman, R.L. Bone density and differential survivorship of fossils classes. J. Anthropol. Archaeol. 1984, 3, $259-299$. [CrossRef]

112. Denys, C. Nouveaux critères de reconnaissance des concentrations de microvertébrés d'après l'étude des pelotes de chouettes du Botswana (Afrique australe). Bull. Mus. Natl. Hist. Nat. A 1985, 7, 879-933.

113. Andrews, P. Owls, Caves and Fossils; Natural History Museum Publications: London, UK, 1990.

114. Fernandez-Jalvo, Y.; Andrews, P. Small Mammal Taphonomy of Gran Dolina, Atapuerca (Burgos), Spain. J. Archaeol. Sci. 1992, 19, 407-428. [CrossRef]

115. Hanquet, C. Évolution des Paléoenvironnements et des Paléoclimats au Pléistocène Moyen, en Europe Méridionale, d'après les Faunes de Micromammifères. Ph.D. Thesis, Université de Montpellier, Montpellier, France, 2011.

116. Desclaux, E.; Hanquet, C.; El Guennouni, K. Origine(s) des accumulations de micromammifères dans quelques sites préhistoriques du Pléistocène moyen et supérieur d’Europe méridionale. In Taphonomie des Petits Vertébrés: Référentiels et Transferts aux Fossils; Laroulandie, V., Mallye, J.-B., Denys, C., Eds.; BAR International Series 2269; Archaeopress: Oxford, UK, 2011; pp. 110-118.

117. Stoetzel, E.; Marion, L.; Nespoulet, R.; Hajraoui, M.E.; Denys, C. Taphonomy and Palaeoecology of the Late Pleistocene to Middle Holocene Small Mammal succession of El Harhoura 2 cave (Rabat-Temara, Morocco). J. Hum. Evol. 2011, 60, 1-33. [CrossRef] [PubMed]

118. Woodthorpe, L. Taphonomie et Paléoécologie des Micromammifères du Dernier Maximum Glaciaire du Site Paléontologique du Coulet des Roches à Monieux (Vaucluse); Master's Thesis, Université de Nice Sophia Antipolis, Nice, France, 2017.

119. Lockie, J.D. The food of the Pine Marten Martes martes in West Ross-Shire, Scotland. Proc. Zool. Soc. Lond. 1961, 136, 187-195. [CrossRef] 
120. Cheylan, G.; Bayle, P. Le régime alimentaire de quatre Mustélidés en Provence: La fouine Martes foina, le blaireau Meles meles, la belette Mustela nivalis et le Putois Putorius putorius. Faune de Provence 1988, 9, 14-26.

121. Andrews, P.; Nesbit-Evans, E.M.N. Small mammal bone accumulation produced by mammalian carnivores. Paleobiology 1983, 9, 289-307. [CrossRef]

122. Chaline, J. Les rongeurs au pilori? Analyse critique des reconstitutions paléoécologiques en microthériodontologie. Bull. AFEQ 1977, 47, 75-81.

123. Chaline, J. Les rongeurs et la climatologie du Pléistocène supérieur. In l'Homme et Son Environnement Pendant le Würm en Europe de l'Ouest; Université de Bordeaux III: Bordeaux, France, 1975; pp. 5-16.

124. Chaline, J. Les rongeurs, les paléoenvironnements et les climats du Pléistocène moyen de Vergranne (Doubs). Annales Scientifiques de l'Université de Franche-Comté-Besançon, Géologie 1983, 5, 31-45.

125. Marquet, J.C. Paléoenvironnement et chronologie des sites du domaine atlantique français d'âge Pléistocène moyen et supérieur d'après l'étude des rongeurs. Ph.D. Thesis, Université de Dijon, Dijon, France, 1989.

126. Desclaux, E. Les petits vertébrés de la Caune de l'Arago à Tautavel (Pyrénées-Orientales): biostratigraphie, paléoécologie et taphonomie. Bulletin du musée d'Anthropologie Préhistorique de Monaco 1992, 35 (Suppl. 4), 9-19.

127. Foury, Y.; Desclaux, E.; Daujeard, C.; Defleur, A.; Raynal, J.P. Évolution des faunes de rongeurs en moyenne vallée du Rhône (rive droite, Ardèche, France) au cours du Pléistocène moyen final et du Pléistocène supérieur ancien du MIS 6 au MIS 4. Quaternaire 2016, 27, 55-79. [CrossRef]

128. Nesbit-Evans, E.M.N.; Van Couvering, J.H.; Andrews, P. Palaeoecology of Miocene sites in Western Kenya. J. Hum. Evol. 1983, 10, 35-48.

129. Valverde, J.A. Remarques sur la structure de l'évolution des communautés de vertébrés terrestres. I. Structure d'une communauté. II. Rapport entre prédateurs et proies. Terre et Vie 1964, 111, 121-154.

130. Legendre, S. Analysis of mammalian communities from the late Eocene and Oligocene of Southern France. Palaeovertebrata 1986, 16, 191-217.

131. Legendre, S. Les communautés de mammifères du Paléogène (Eocène supérieur et Oligocène) d'Europe occidentale: Structures, milieux et évolution. Münch. Geowiss.e Abhand. (A). Geologie und Palaeontologie $1989,16,1-110$.

132. Desclaux, E. Les petits vertébrés de la Caune de l'Arago à Tautavel (Pyrénées-Orientales). Paléontologie, Paléoécologie et Taphonomie. Ph.D. Thesis, Muséum National d'Histoire Naturelle de Paris, Paris, France, 1992.

133. Desclaux, E. Essai de reconstitution des paléoenvironnements par la méthode des cénogrammes. In Le Gisement Paléolithique Moyen de la Grotte des Cèdres (Le Plan d'Aups, Var); Defleur, A., Crégut-Bonnnoure, E., Eds.; DAF: Paris, France, 1995; pp. 156-157.

134. Montuire, S.; Desclaux, E. Palaeoecological analysis of mammalian faunas and environmental evolution in the South of France during the Pleistocene. Boreas 1997, 26, 355-365. [CrossRef]

135. Montuire, S.; Desclaux, E. Analyse paléoécologique des faunes de mammifères et évolution des environnements dans le Sud de la France au cours du Pléistocène. Quaternaire 1997, 8, 13-20. [CrossRef]

136. Hewitt, G. Some genetic consequences of ice age, and their role in divergence and speciation. Biol. J. Linn. Soc. 1996, 58, 247-276. [CrossRef]

137. Hewitt, G. Post-glacial recolonization of European biota. Biol. J. Linn. Soc. 1999, 68, 87-112. [CrossRef]

138. Hewitt, G. The genetic legacy of the Quaternary ice ages. Nature 2000, 405, 907-913. [CrossRef] [PubMed]

139. Santucci, F.; Emerson, B.C.; Hewitt, G. Mitochondrial phylogeography of european hedgehogs. Mol. Ecol. 1998, 7, 1163-1172. [CrossRef] [PubMed]

140. Stewart, J.; Lister, A.; Barnes, I.; Dalen, L. Refugia revisited: Individualistic responses of species in space and time. Proc. R. Soc. Lond. B 2010, 277, 661-671. [CrossRef] [PubMed]

141. Schmitt, T. Molecular biogeography of Europe: Pleistocene cycles and postglacial trends. Front. Zool. 2007, 4, 1-13. [CrossRef] [PubMed]

(C) 2018 by the authors. Licensee MDPI, Basel, Switzerland. This article is an open access article distributed under the terms and conditions of the Creative Commons Attribution (CC BY) license (http:/ / creativecommons.org/licenses/by/4.0/). 